\title{
Inferring Thalamocortical Monosynaptic Connectivity In-Vivo
}

\author{
Yi Juin Liew ${ }^{1}$, Aurélie Pala ${ }^{1}$, Clarissa J Whitmire ${ }^{1}$, William A Stoy ${ }^{1}$, \\ Craig R Forest ${ }^{1,2}$, Garrett B Stanley ${ }^{1}$ \\ ${ }^{1}$ Wallace H Coulter Department of Biomedical Engineering, Georgia Institute of \\ Technology and Emory University, Atlanta, GA, USA \\ ${ }^{2}$ George W Woodruff School of Mechanical Engineering, Georgia Institute of \\ Technology, Atlanta, GA, USA
}

Abbreviated title: Inferring monosynaptic connectivity in vivo

With 44 text pages, 8 figures, 6 supplemental figures

Correspondence: Garrett B Stanley

Coulter Department of Biomedical Engineering

Georgia Institute of Technology \& Emory University

313 Ferst Dr

Atlanta GA 30332-0535, USA
Keywords: $\quad$ thalamocortical; cross correlation; signal detection; causality; inference

Phone: $404-385-5037$

Fax: 404-385-5044

Email: garrett.stanley@bme.gatech.edu

Disclosures: The authors declare no competing financial interests.

Acknowledgements: This work was supported by $\mathrm{NIH} / \mathrm{NIMH}$ Brain Initiative Grant U01MH106027 (GBS and CRF) and NIH/NINDS Brain Initiative Grant R01NS104928 (GBS). YL was supported by a Georgia Tech-Emory-PKU Global Biomedical Engineering Fellowship. AP was supported by a postdoctoral fellowships P300PA 177861 and P2ELP3_168506 from the Swiss National Science Foundation (SNSF). CJW was supported by an NIH NRSA Pre-doctoral Fellowship. WAS is supported by an NSF Graduate Research Fellowship. 


\section{Abstract/Summary:}

As the tools to simultaneously record electrophysiological signals from large numbers of neurons within and across brain regions become increasingly available, this opens up for the first time the possibility of establishing the details of causal relationships between monosynaptically connected neurons and the patterns of neural activation that underlie perception and behavior. Although recorded activity across synaptically connected neurons has served as the cornerstone for much of what we know about synaptic transmission and plasticity, this has largely been relegated to ex-vivo preparations that enable precise targeting under relatively well-controlled conditions. Analogous studies invivo, where image-guided targeting is often not yet possible, rely on indirect, data-driven measures, and as a result such studies have been sparse and the dependence upon important experimental parameters has not been well studied. Here, using in-vivo extracellular single unit recordings in the topographically aligned rodent thalamocortical pathway, we sought to establish a general experimental and computational framework for inferring synaptic connectivity. Specifically, attacking this problem within a statistical signal-detection framework utilizing experimentally recorded data in the ventral-posterior medial (VPm) region of the thalamus and the homologous region in layer 4 of primary somatosensory cortex (S1) revealed a trade-off between network activity levels needed for the data-driven inference and synchronization of nearby neurons within the population that result in masking of synaptic relationships. Taken together, we provide a framework for establishing connectivity in multi-site, multi-electrode recordings based on statistical inference, setting the stage for large-scale assessment of synaptic connectivity within and across brain structures.

\section{New \& Noteworthy:}

60 Despite the fact that all brain function relies on the long-range transfer of information 61 across different regions, the tools enabling us to measure connectivity across brain 62 structures are lacking. Here, we provide a statistical framework for identifying and 63 assessing potential monosynaptic connectivity across neuronal circuits from population 64 spiking activity that generalizes to large-scale recording technologies that will help us to 65 better understand the signaling within networks that underlies perception and behavior. 


\section{Introduction}

Every aspect of brain function, from sensory and motor processing, to memory and cognition, involves complex circuitry and communication across many different brain areas. Despite this fact, what we know about brain function has been derived largely from electrophysiological recordings targeted at single regions, and upon gross anatomical connection patterns across brain regions without specific precise knowledge of synaptic connectivity. Ultimately, understanding the causal interaction of neuronal dynamics that underlie perception and behavior requires ground truth evidence for synaptic connectivity that necessitates intracellular access to both pre- and post-synaptic neurons. Despite exciting advancement of a range of recording technologies such as simultaneous multi-neuron intracellular recordings in-vivo (Jouhanneau and Poulet 2019; Kodandaramaiah et al. 2018) and deep structure targeted patching (Stoy et al. 2017), targeting connections in-vivo using intracellular approaches remains a labor-intensive endeavour, is ultimately limited to a very small number of neurons, and does not scale to the circuit level. Extracellular recordings offer solutions to some of these issues, and thus offer promise in this direction.

The field of neuroscience is now at a time where large-scale recordings of neuronal populations at cellular resolution are possible across brain structures, due to the development of multi-electrode recording technologies such as 'Neuronexus' (Berényi et al. 2014; Buzsáki et al. 2015) (NeuroNexus Inc.), 'Neuropixels' (Jun et al. 2017), 'NeuroSeeker' (Fiáth et al. 2018; Raducanu et al. 2016)), and 3D silicon probes (Rios et al. 2016). These technologies provide access to surveying network level information flow, driving a growing need for a rigorous experimental and analytic framework to identify functional relationships across brain structures. In previous studies, in-vivo approaches to establishing synaptic connectivity across recorded pairs of neurons have been developed based on analytic methods applied to recorded spiking data (Nowak and $\mathrm{J}$ 2000; Perkel et al. 1967), historically conducted in larger animals such as cats, rabbits, and rats (Reid and Alonso 1995; Swadlow and Gusev 2001; Wang et al. 2010) and only more recently in mouse (Lien and Scanziani 2018). Approaches based on spike correlations are the most common for quantifying functional interactions and inferring monosynaptic connections in-vivo. These approaches have been utilized in paired

101 recording studies that involved measuring correlation in the spiking activity between neurons (Barthó et al. 2004; Csicsvari et al. 1998; English et al. 2017; Fujisawa et al.

103 2008; Reid and Alonso 1995; Swadlow and Gusev 2001; Wang et al. 2010) or between

104 the spiking activity of a pre-synaptic neuron and the subthreshold membrane potential of 105 a putative post-synaptic target (Bruno and Sakmann 2006; Matsumura et al. 1996) 106 (London et al. 2010; Sedigh-Sarvestani et al. 2017; Yu and Ferster 2013). Correlational 
107 approaches are statistical in nature and thus have been anecdotally reported to be

108 strongly dependent upon data length and are very sensitive to a range of possible

109 confounds, but a comprehensive understanding of these relationships and a unified

110 approach are both lacking.

112 Here, using the thalamocortical circuit in rodents as a model system, we establish 113 methodological strategies for extracellular, topographically aligned in-vivo single-unit 114 recordings in thalamus and cortex for both rat and mouse. This analytic framework for 115 inferring connectivity is based on signal-detection theory, and directly addresses issues 116 of data length dependence and confounds produced by population synchrony. 117 Specifically, we outline a work-flow of topographic mapping and histological validation, 118 followed by a statistical approach for signal-detection based classification of putative 119 connected and non-connected pairs with an assessment of classification confidence that 120 is scalable to the large-scale recording approaches that are emerging in the field. We 121 found that while the amount of spiking was a strong determinant of the accuracy of the 122 inference of connectivity, there was an important tradeoff between the activity in the 123 network and the underlying population synchrony that regulates the likelihood of both 124 discovering a synaptic connection that is present and correctly classifying unconnected 125 pairs as such. Taken together, we provide a data-driven framework for inferring 126 connectivity and the corresponding statistical confidence that generalizes to large-scale 127 recordings across brain structures. 
130

131

132

133

134

135

136

137

138

139

140

141

142

143

144

145

146

147

148

149

150

151

152

153

154

155

156

157

158

159

160

161

162

163

164

165

166

167

168

\section{METHODS}

\section{Animals}

Twelve female adult Sprague Dawley rats (no age restriction, 225-330g) and four adult male C57BL/6 mice (8-16 weeks, 25-35g) were used in all the experiments. All procedures were approved by the Georgia Institute of Technology Institutional Animal Care and Use Committee and followed guidelines established by the National Institutes of Health. Note that there were some major differences in animal preparation, surgery and craniotomies as well as electrophysiology for rat and mouse, as described below. However, procedures such as whisker stimulation, post-mortem histology, and analytical methods used for analysis were largely identical.

\section{Animal preparation, surgery and craniotomies.}

Rat. Animals were first sedated with $5 \%$ vaporized isoflurane and maintained at $3 \%$ vaporized isoflurane when transitioned to fentanyl-cocktail anesthesia via tail vein injection. Fentanyl cocktail anesthesia (fentanyl [5 $\mu \mathrm{g} / \mathrm{kg}$ ], midazolam [2 $\mathrm{mg} / \mathrm{kg}$ ], and dexmedetomidine [150 mg/kg])) was administered continuously via a drug pump at an initial rate of $4.5 \mathrm{uL} / \mathrm{min}$ (Ahissar et al. 2000; Whitmire et al. 2016). During the transition, anesthesia level was monitored closely by measurement of heart rate and respiratory rate. Body temperature was maintained at $37^{\circ} \mathrm{C}$ by a servo-controlled heating pad. Under the effect of both anesthetic agents, the animal's heart rate tended to decrease gradually over several minutes. The isoflurane level was then titrated down in $0.5 \%$ decrements during the transition to the fentanyl cocktail. Upon successful transition, which ranged from 5-15 min depending on the animal, the heart rate was targeted for approximately $240-270$ bpm. After the effects of fentanyl cocktail stabilized (in terms of heart rate) and the animals showed no toe pinch response, the animals were then fixed in the stereotaxic device by securing the head of the animal in place with ear bars on a floating table in an electromagnetically shielded surgery suite. The position of the ear bars was verified with uniform eye levels and usually with eardrum penetration on both sides of the head. Eye ointment (Puralube Vet Ointment) was applied to prevent the animal's eyes from dehydration. An incision was made along the midline of the skull, and skin was removed for visibility of bregma and lambda (Paxinos and Watson 2007). Connective tissue and muscle that were close to the ridge of the skull were detached and removed to expose the skull surface that was directly above the barrel cortex. To ensure that the skull remained level throughout the recording, the height of the skull surface was measured at bregma and lambda, and the difference was minimized $(<200 \mathrm{um}$ ) by adjusting the angle of the head with the nose cone position.

We made two craniotomies on the animal's left hemisphere above the ventroposteromedial nucleus (VPm) and the S1 barrel cortex based on stereotaxic coordinates. 
169 For the cortical craniotomy, which usually extended over the ridge of the skull, the 170 stereotaxic device was rotated to an angle ( $40^{\circ}$ relative to vertical) for better visualization 171 and drilling. Both craniotomies were drilled slowly until the skull piece appeared to be 172 floating and mobile. We irrigated the skull surface periodically with Ringer's solution when 173 drilling to remove debris and prevent overheating from drilling. Before removing the skull

174 piece to expose the cortex, a small dental cement reservoir was carefully built around the 175 craniotomies to hold the Ringer's solution for irrigation and to keep the recording site 176 continually moist. The lateral side of the wall was built thicker and higher due to the 177 curvature of the skull toward the ridge. To provide strong adherence to the skull, 178 cyanoacrylate containing adhesive (Krazy Glue, Elmer's Products Inc.) was carefully 179 applied around the external edges of the reservoir, leaving the bregma and major skull 180 sutures visible at all times. For grounding purposes, another hole with $\sim 0.5 \mathrm{~mm}$ diameter 181 was drilled (Henry Schein, Carbide Burr HP 2) on the right hemisphere of the skull and 182 fastened with a skull screw and a metal wire. The skull pieces at both recording sites 183 were then carefully removed with forceps (FST Dumont \#5/45). To minimize brain 184 swelling, the dura was left intact on both recording surfaces, and warm Ringer's solution 185 was repeatedly added and absorbed with cotton tips until the blood was cleared. An 186 absorbent gelatin compressed sponge (Gelfoam, Pfizer Inc.) was sometimes used to 187 clear the blood.

Mouse. Head-plate implantation and intrinsic imaging procedure were usually performed 190 at least three days to one week before acute experiment. A lightweight custom metal 191 (titanium or stainless steel) head-plate was implanted on the skull of the mouse for head 192 fixation and improved stability during recording, in accordance with a previously described 193 protocol (Borden et al. 2017). During this survival surgical preparation, the animal was 194 sedated with 5\% vaporized isoflurane and anesthesia was maintained with 2-3\% 195 isoflurane for the head-plate procedure. We administered opioid and non-steroidal anti196 inflammatory analgesic (SR-Buprenorphine $0.8-1 \mathrm{mg} / \mathrm{kg}$, SC, pre-operatively, and 197 Ketoprofen 5-10 mg/kg, IP, post-operatively) and covered the animal's eyes with 198 ophthalmic eye ointment (Puralube Vet Ointment). Body temperature was monitored and 199 maintained at $37^{\circ} \mathrm{C}$. After sterilizing the skin above the skull and applying topical 200 anesthetic (lidocaine $2 \%, 0.05 \mathrm{ml}$, max $7 \mathrm{mg} / \mathrm{kg}, \mathrm{SC}$ ), we removed the skin and scraped 201 off periosteum and any conjunctive tissue off the skull. We gently separated tissue and 202 muscles close to the lateral edge of the skull using a scalpel blade (Glass Van \& 203 Technocut, size no. 15), leaving sufficient room for head-plate attachment and away from 204 targeted recording areas. To ensure that the skull surface remained level during head 205 fixation, we adjusted animal's head position to minimize the relative height difference $(<$ $206150 \mathrm{um}$ ) between the skull surface at the bregma and lambda landmarks (Franklin and 207 Paxinos 2008). We found this step to be critical especially for VPm targeting. We secured 
208 the metal headplate over the intact skull with C\&B-Metabond (Parkell Prod Inc) and skin 209 adhesive (Loctite 401, Henkel). The Metabond dental acrylic was chilled using ice, slowly 210 applied to the skull surface, and allowed to cure for 5-10 minutes before we covered the 211 rest of the attachment site and edges of the skin incision with skin adhesive (Loctite 401) 212 to ensure that the skin edges were securely adhered to the skull. The final head-plate and 213 dental acrylic structure created a recording well for holding Ringer solution (in mM: 135 $214 \mathrm{NaCl}, 5 \mathrm{KCl}, 5 \mathrm{HEPES}, 1 \mathrm{MgCl}_{2}-6 \mathrm{H}_{2} \mathrm{O}, 1.8 \mathrm{CaCl}_{2}-2 \mathrm{H}_{2} \mathrm{Ol} \mathrm{pH} \mathrm{7.3)} \mathrm{for} \mathrm{future} \mathrm{imaging} \mathrm{and}$ 215 electrophysiological recording sessions. For grounding purposes, another hole with $\sim 0.5$ $216 \mathrm{~mm}$ diameter was drilled (Henry Schein, Carbide Burr HP 2) on the right hemisphere of 217 the skull and fastened with a skull screw (Miniature Self-Tapping Screws, J. I. Morrisco). 218 A metal wire was connected to the skull screw on the day of recording to serve as animal 219 ground. We applied a thin layer of transparent glue (Loctite 401, Henkel) over the left 220 hemisphere to protect the skull and covered the exposed skull with a silicone elastomer 221 (Kwik-Cast, World Precision Instruments) if there were no other additional procedures 222 after head-plate implantation.

223

224 Intrinsic Optical Imaging in the Mouse S1 Barrel Cortex. We performed intrinsic signal optical imaging of whisker-evoked responses in mouse primary somatosensory cortex under $1-1.2 \%$ isoflurane anesthesia to functionally identify individual barrel columns (Lefort et al. 2009; Pala and Petersen 2015). All whiskers except the whiskers of interest (A2, B1, C2, D1, D3 and E2) were trimmed. We thinned the skull using a dental drill (0.1 $\mathrm{mm}$ diameter bit, Komet, USA) until blood vessels became visible. We applied warm Ringer's solution on top of the skull and covered it with a glass coverslip (thickness 0.13$0.17 \mathrm{~mm}$, Fisherbrand). We captured a reference image of the blood vasculature under green illumination (530nm) (M530F1 LED, Thorlabs). We delivered repetitive whisker stimuli $(10 \mathrm{~Hz}$, sawtooth pulses, $1000 \mathrm{deg} / \mathrm{s}$ ) to individual whiskers using a galvanometer system (Cambridge Technologies) while performing imaging on barrel cortex under red illumination (625 nm) (M625F1 LED, Thorlabs) - see Whisker Stimulation section below. Images were acquired using a CCD Camera (MiCam02HR, SciMedia) at $10 \mathrm{~Hz}$, with a field of view of approximately $4 \times 2.5 \mathrm{~mm}$, corresponding to $384 \times 256$ pixels (resolution: 100pixel/mm). Each trial lasted $10 \mathrm{~s}$, with $4 \mathrm{~s}$ of baseline followed by $4 \mathrm{~s}$ of whisker stimulation and $2 \mathrm{~s}$ without stimulation. The inter-trial interval was 30 s. Whisker-evoked responses were averaged over 10-20 trials. Intrinsic signals were measured as the relative change in reflectance by taking the overall mean reflectance during whisker stimulation ( $\left.R_{\text {stim }}\right)$ and subtracted the mean baseline reflectance ( $R_{\text {stim }}$ - $R_{\text {baseline }}$ ). Acquisition and processing of the images were implemented using "BV_Ana" software (MiCam02HR, SciMedia, Ltd) and Matlab (MathWorks, Natick, 2015). We recorded intrinsic signals from at least three barrels and estimated location of the unmapped barrels by overlaying a template barrel map reconstructed from histology. At the end of the 
247 imaging session, we applied transparent glue (Loctite 401) directly on the S1 site to

248 protect the thinned skull from contamination and infection and sealed the exposed skull with a silicone elastomer (Kwik-Cast, World Precision Instruments). and animal should remain in the flat skull position. We covered the eyes with ophthalmic eye ointment (Puralube Vet Ointment) to prevent dehydration. The animal was then anesthetized using 2-3\% isoflurane. Before performing the craniotomies, we thinned the skull above VPm and S1 layer-by-layer with a dental drill $(0.1 \mathrm{~mm}$ diameter bit, Komet, USA) and irrigated the skull surface with Ringer's solution frequently to remove debris and prevent overheating. When the skull was thin enough to easily puncture, we made two small craniotomies above VPm (approximately $1 \mathrm{~mm}$ diameter; centered at $1.8 \mathrm{~mm}$ caudal and $1.8 \mathrm{~mm}$ lateral to bregma) and $\mathrm{S} 1$ (approximately $0.5 \mathrm{~mm}$ diameter, using the intrinsic imaging signal and the blood vasculature as a landmark) by penetrating the thinned skull using an insulin syringe needle tip (at about 40-50 degree angle) to make small holes outlining the edges of the craniotomies until the circular skull piece was loosely attached. The less perforated region of the circular skull piece was used as a pivot, and we levered the skull pieces off from the more perforated edge using fine-tip forceps (FST Dumont \#5SF) to expose the brain surface.

\section{Electrophysiology}

268 Neuronal recordings. Tungsten microelectrodes (FHC, impedance: 2-5 M $2,75 \mu \mathrm{m}$ in diameter) were used in thalamus and cortex to isolate single units that were responding to a single primary whisker on the contralateral side of the face. Multielectrode silicon probes with 32 recording sites (A1×32-Poly3-5mm-25s-177 or A1x32-Poly2-10mm-50s177, NeuroNexus) were also independently lowered into the thalamus and cortex. To improve signal quality, we electroplated the silicon probes with the polymer PEDOT:PSS (Baião 2014) with a nanoZ device (Multi Channel Systems, Germany). The impedances of the contact sites were measured prior to each recording and ranged from 0.3-0.8 MOhms. After each use, the contact sites were soaked in $20 \%$ Contrad (labware detergent) overnight for cleaning and the impedances usually returned to the initial values (0.3-0.8 MOhms). The impedances for the silicon probes were always measured prior to a recording session and contact sites that were defective usually showed large fluctuations in raw voltage traces and signals detected at these sites were excluded for analysis prior to spike-sorting. Note that tungsten microelectrodes were used in rat studies and probe recordings were used in mouse studies. Data were collected using a 64-channel electrophysiological data-acquisition system (TDT Model, etc. RZ2 Bioprocessor). Neuronal signals were amplified, and bandpass filtered $(500 \mathrm{~Hz}-5 \mathrm{kHz})$ and digitized at $\sim 25 \mathrm{kHz}$ per channel. Stimulus waveform and other continuous data were 
286

287

288

289

290

291

292

293

294

295

296

297

298

299

300

301

302

303

304

305

306

307

308

309

310

311

312

digitized at $10 \mathrm{kHz}$ per channel. Simultaneously, the local field potential (LFP) signals were obtained by using a $0.5-200 \mathrm{~Hz}$ bandpass filter. The LFPs were used to identify primary whisker response (see Whisker Stimulation).

Rat. VPm recordings. We targeted the VPm region of the thalamus by advancing a single tungsten microelectrode (FHC, 2 MOhm impedance) perpendicular to the pial surface into the thalamic craniotomy centered at $3.0 \mathrm{~mm}$ caudal and $3.0 \mathrm{~mm}$ lateral to bregma. We quickly advanced ( $50 \mathrm{um} / \mathrm{s})$ to the depth of $4000 \mu \mathrm{m}$ and slowed down to the speed of $\sim 3 \mu \mathrm{m} / \mathrm{s}$ while searching for responsive cells by manually deflecting the whisker on the contralateral side of the vibrissal pad. Whisker responsive cells were typically located at a depth of $4800-5300 \mu \mathrm{m}$, measured using a precision micromanipulator (Luigs \& Neumann, Germany). S1 recordings. At a position $5.8 \mathrm{~mm}$ lateral and $2.5 \mathrm{~mm}$ caudal to bregma, we inserted a single tungsten microelectrode at an angle of $40-45^{\circ}$ (relative to the vertical axis of electrode holder). We positioned the tip of the electrode gently touching the dura and advanced slowly to create an opening in the membrane. The microelectrode was then slowly advanced into cortical tissue at a speed of $\sim 3 \mu \mathrm{m} / \mathrm{s}$ (measured at $1 \mu \mathrm{m}$ resolution). All cortical units were recorded at stereotaxic depths of $700-1000 \mu \mathrm{m}$, corresponding to layer IV of rat barrel cortex based on literature (Bruno and Sakmann 2006; Constantinople and Bruno 2013).

Mouse. VPm recordings. For first penetration, electrode was typically lowered at $1.8 \mathrm{~mm}$ caudal and $1.8 \mathrm{~mm}$ lateral to bregma. If we could not functionally locate VPm using this method, we used the relative distance from the location of barrel cortex (usually measured $1.0-1.2 \mathrm{~mm}$ from the B1 or Beta barrel column to find whisker responsive thalamic regions). For probe recordings, we searched for whisker responsive cells by manual whisker deflection using the deepest channel as reference. We typically found whisker responsive cells were at a depth between 2800 um-3200 um. S1 recordings. Based on intrinsic imaging signal, a small craniotomy (diameter $\sim 0.5 \mathrm{~mm}$ ) was created at the desired location on the barrel cortex that was distant from blood vessels. We positioned the electrode at a $35^{\circ}$ angle from the vertical axis (parallel to the barrel column) and recorded from cortical neurons at a stereotaxic depth of 300-500um from the cortical surface.

\section{Whisker Stimulation}

To identify the single whisker of interest, we first manually deflected all whiskers on the vibrissal pad while monitoring the extracellular signal. Once units were isolated by moving the electrode as close as possible to the responsive cells that primarily responded to single whisker, we delivered controlled, single whisker stimulation in the rostral-caudal plane using a computer-controlled actuator, galvo-motor (galvanometer optical scanner model $6210 \mathrm{H}$, Cambridge Technology). Whiskers were trimmed $\sim 12 \mathrm{~mm}$ from the face. 
325 Primary whisker (PW) was defined as whisker deflection that evoked the maximal 326 neuronal response with shortest latency (see Transient Stimulus). The primary whisker 327 was fed into the insertion hole at the end of an extension tube (inner diameter: 200-300 $328 \mu \mathrm{m}$ um, length: $15 \mathrm{~mm}$ ) that was connected to the rotor of the galvanometer stimulator. 329 The stimulus probe was positioned at $10 \mathrm{~mm}$ from the vibrissal pad. The range of motion 330 of the galvanometer was $\pm 20^{\circ}$, with a bandwidth of $200 \mathrm{~Hz}$. The galvanometer system was 331 controlled using a custom developed hardware/software system (Matlab Real-Time 332 Simulink System, MathWorks). Whisker evoked responses were measured using raster 333 plots and peri-stimulus time histograms (PSTH, 1-ms bin resolution) across trials.

Transient stimulus. For mapping whisker receptive field at the recording sites, we presented high velocity (600 or $1000 \%$ s) rostral-caudal whisker deflection to evoke reliable whisker responses. Trains of solo pulses, followed by a separate, pulsatile adapting stimulus at 8-10 Hz (Borden et al. 2017; Whitmire et al. 2016) were delivered for 60-100 times. To measure whisker response to different stimulus strength, pulses with angular deflection velocities of 50,125,300, 600, 900, $1200 \mathrm{deg} / \mathrm{s}$ were presented randomly. Rostral-caudal pulse deflections were either a Gaussian-shaped deflection waveform or a simple exponential sawtooth (rise and fall time $=8 \mathrm{~ms}$ ). We quantified whisker evoked activity $30 \mathrm{~ms}$ after stimulus onset and then further characterized onset latencies of first spike within the response window in response to non-adapting solo pulses and adapting pulsatile stimuli over 60-100 trials. Mean first spike latencies, defined as the average time delay between stimulus onset and the first spike in the response window after stimulus presentation. This metric was used for all thalamic and cortical units. For 32-channel probe recording in thalamus, we quantified the LFP response in a $30 \mathrm{~ms}$ post-stimulus window for each channel to identify principal whiskers (PW). Well-isolated single units that showed whisker responsiveness was verified by calculating LFP peak amplitude and peak latency to different whisker stimulation.

A neuronal pair was verified to be topographical aligned when they (1) shared maximum

354 response (SUs max FR and LFP amplitude) to the same primary whisker under punctate 355 stimulation and (2) latency difference of whisker response within 1-5 ms delay (LFP peak 356 latency and SU mean FSL, or SU mean peak latency).

Weak sinusoidal stimulus. For monosynaptic connection quantification, we probed the 359 thalamocortical circuit using a weak, desynchronizing sinusoidal whisker stimulus to 360 elevate baseline firing rates in recorded units. We delivered $4^{\circ}, 2-4 \mathrm{~Hz}$ weak sinusoidal 361 deflections (Bruno and Sakmann 2006; Bruno and Simons 2002; Wang et al. 2010) for 362 approximately 200-500 trials to obtain at least 2000 spikes for the cross-correlation 
363 analysis. Occasionally, we eliminated the first $0.5-1 \mathrm{~s}$ of the trials due to high firing rate at the onset of stimulus presentation.

Post-mortem Histology In order to verify recording sites and the angle of penetration that was optimal for locating VPm and S1, we perfused a small subset of animals after the paired recording experiment. To label each electrode recording track, we slowly retracted the electrode along its axis of entry at the conclusion of recording and applied a few drops of Dil $(2.5 \mathrm{mg} / \mathrm{mL}$, in ethanol). We then reinserted the electrode into the same penetration site and back along the same axis and left the electrode in the brain for at least 15 minutes. Following the end of the experiment, we euthanized the animal with an overdose of sodium pentobarbital (euthasol, $0.5 \mathrm{~mL}$ at $390 \mathrm{mg} / \mathrm{mL}$ for rat, $0.1 \mathrm{~mL}$ at $390 \mathrm{mg} / \mathrm{mL}$ ), performed a transcardial perfusion, extracted the brain, and fixed the brain in 4\% paraformaldehyde (PFA, Electron Microscopy Sciences) overnight. We sliced the brain in 100 um coronal sections and performed cytochrome oxidase staining to reveal VPm (barreloid) and S1 (barrel). As an additional verification of recording site, we identified the overlap between the Dil stained recording track and the CO-stained regions.

\section{Analytical Methods} Extracellular spike sorting. In assessing potential synaptic connectivity using crosscorrelation analysis, the clear isolation of single unit activity from extracellularly recorded voltage signals is particular critical. In all of our paired recording experiments, we performed extracellular single-unit recordings using either Tungsten microelectrodes or 32-channel NeuroNexus probes. Although similar in approach, the sorting of recorded data into clusters were implemented using different software. For the single microelectrodes, we performed spike sorting offline using the Plexon Offline Sorter (Plexon Inc, Dallas TX, USA), while for the 32-channel probe recordings we utilized KiloSort2 software package (https://github.com/MouseLand/Kilosort2) (Harris et al. 2000). For high density probe recordings, there was an additional manual curation step using phy 392 (https://github.com/cortex-lab/phy) where we refined the output of automatic algorithm and determined if merging or splitting of specific clusters were necessary based on refractory violation, waveform shape as well as cross-correlogram between clusters. For both Tungsten microelectrode and high-density probe recordings, we classified the clusters as single- or multi-units based on the signal-to-noise ratio and inter-spike interval (ISI) distribution. We only included well-isolated clusters for further analysis. The selection of well-isolated clusters, or single units, was based on two criteria: (1) high signal-to-noise ratio (SNR) of the spike waveform: Peak-to-peak amplitude (Vpp) of spike waveforms 400 greater than three standard deviation (SD) of the waveform. (2) Had a clear refractory 401 period(Buzsáki 2004; Fiáth et al. 2019). We defined a single-unit as well-isolated if it had 
SNR greater than 3, ISI violation less than $1 \%$ for cortical unit and $2 \%$ for thalamic unit and spike waveform with a peak-to-peak amplitude (Vpp) greater than $60 \mu \mathrm{V}$. Given that the spikes of the same neuron were usually detected on multiple sites of a 32-channel probe, the peak-to-peak amplitude and standard deviation of the waveform were computed using channel that had the largest amplitude of spike waveform. another, and particularly so in the mouse, where the regions are very small and very close together. After recording, we used a combination of measures to classify thalamic units as VPm: (1) Average first-spike latency to punctate (non-adapting) stimuli, quantifying the average time between stimulus onset and the first spike fired during the neural response window (30 ms after stimulus onset) (Storchi et al. 2012). (2) Shift in average first-spike latency response to adapting stimuli, defined as the time difference between average response to the first adapting stimulus and last adapting stimulus (Masri et al. 2008). (3) Response reliability, defined as the percentage of trials where a response was detected within $20 \mathrm{~ms}$ to repetitive stimulation $(8-10 \mathrm{~Hz})$ (Mainen and Sejnowski 1995). We excluded thalamic units with average first-spike latency of more than $12 \mathrm{~ms}$ (see Supplementary Figure $2 \mathrm{~A}$ ), latency shift of more than $20 \mathrm{~ms}$, or response reliability of less than $20 \%$. recipient from VPm projections and there exist some heterogeneity in terms of cortical cell types in Layer IV (Bruno and Simons 2002). Here, we classified cortical units into fast-spiking unit (FSU) and regular-spiking unit (RSU) by using waveform parameter. We quantified the time interval between trough to peak ( $\mathrm{t}$ 2p) of the spike waveform (Barthó et al. 2004).

Cross-correlation analysis. All analysis was performed in a trial-by-trial basis. Given a spike in a 'reference' neuron, we computed the relative times of the spikes from a 'target' neuron that occurred within a 25-millisecond window before and after each reference spike. Cross-correlograms were constructed using a 0.5 milliseconds bin. Traditionally, monosynaptic interactions were known to produce short latency peaks in the crosscorrelograms. The latency of the monosynaptic peak within cross-correlograms was typically reported to center around $2.5 \mathrm{~ms}$, estimated from thalamocortical EPSPs and spiking activity (Alonso and Martinez 1998; Alonso et al. 2001; Bruno and Sakmann 2006; Bruno and Simons 2002; Reid and Alonso 1995; Sedigh-Sarvestani et al. 2017; Swadlow et al. 1978; Swadlow 1989; 2003; Swadlow and Gusev 2001). To ensure our analysis captures correlation from monosynaptic delays only, we allowed for $1.5 \mathrm{~ms}$ jitter on each side, setting the lower bound to be $1 \mathrm{~ms}$ and the upper bound to be $4 \mathrm{~ms}$. This eliminated peaks that could arise in the $0-1 \mathrm{~ms}$ bin due to a shared common input, as well as 
441 disynaptic EPSPs that could have latencies longer than approximately $5 \mathrm{~ms}$ (Gil and 442 Amitai 1996). Here, for the monosynaptic connectivity inference, we used thalamic spiking 443 as the reference and cortical spiking as the target and examined correlated firing within a 444 lag window of 1-4 ms. The results here were relatively invariant to the specific choice over 445 a range of lag windows, but we found the $1-4 \mathrm{~ms}$ window to be the most conservative (i.e. 446 smaller window than 1-5 ms but resulted in same number of pairs classified as 447 "connected", see Supplementary Figure 3). For the spontaneous condition, we 448 segmented the spike train data into 5s-trials (matching duration of stimulus-based trials) 449 and performed cross-correlation analysis in a trial-by-trial manner. To measure the level 450 of presynaptic synchronization in thalamus, we performed a cross-correlation analysis 451 and computed synchrony strength using a central area under the cross-correlogram for 452 all thalamic pairs that responded to the same principal whisker. The principal whisker was 453 identified using the functional responses as measured by the local field potential (LFP) 454 and in the single unit (SU) data. Thalamic synchrony was computed for each thalamic 455 pair using the total number of spikes within a $15 \mathrm{~ms}$ ( $\pm 7.5 \mathrm{~ms}$ ) window of the cross456 correlogram $\left(\mathrm{N}_{\mathrm{cc}}\right)$, normalized by the mean number of spikes from each neuron ( $\mathrm{N}_{\text {ref, }}$ 457 Ntarget) (Alonso and Martinez 1998; Bruno and Sakmann 2006; Temereanca et al. 2008; 458 Wang et al. 2010; Whitmire et al. 2016):

Probabilistic measure of connectivity inference. For each pair of thalamocortical neurons, we repeated the cross-correlational analysis with bootstrapping method. This was done by performing resampling on the dataset with replacement, with approximately 500-1000 iterations for each condition. By counting the number of iterations that fulfill the criteria for classifying the monosynaptic connection, this resulted in a p-value that can be attached to the binary classification of monosynaptic connection (see Results: Inferring connectivity in the context of a signal detection framework). We referred to this $p$-value as the probability of inferring monosynaptic connection, P(Inferring 'connected'). implemented by using subsamples (in unit of trials) of VPm and S1 spikes for crosscorrelation analysis. For each data-length condition, we computed the resampled peak

474 height, $h^{*}$. A bootstrapped estimator of bias was computed as the difference between the 475 mean of the resampled peak height and the original metric, assuming a normal 476 distribution: 


$$
\text { bias }=E\left(h^{*}-h\right)
$$

Where $E($.$) denotes statistical expectation. The variance was estimated as the square of$ the resampled peak height:

$$
\text { variance }=\operatorname{var}\left(h^{*}\right)
$$

A significance level for the inference for a 'putatively connected' pair was computed and denoted as the probability of a hit when the peak height metric for the resampled data exceeded the criterion for the inference of a functional connection. The probability of a miss is complementary:

$$
P(\text { hit })=\frac{\#\left\{h^{*} \geq \text { criterion }\right\}}{\# \text { of iterations }}, \quad \text { criterion }=3.5
$$

$$
P(\text { miss })=1-P(\text { hit })
$$

On the other hand, the significance level for a 'not connected' pair was computed and denoted as the probability of a correct reject when the peak height metric for the resampled data is less than the criterion.

$$
P(\text { correct reject })=\frac{\#\left\{h^{*}<\text { criterion }\right\}}{\# \text { of iterations }}, \text { criterion }=3.5
$$

$$
P(\text { false alarm })=1-P(\text { correct reject })
$$

Thalamic synchrony effect on connectivity metric. Simulation for various thalamic synchrony level was implemented by manipulating both firing rate and spike timing of $\mathrm{VPm}$ and S1. Given that both firing rate and thalamic synchrony were affected with increasing stimulus strength, our simulation was conducted using data that was collected under spontaneous condition. For a connected pair, we set the ratio between VPm and S1 firing to be constant at each synchrony level for simplification. However, we systematically increased spiking activity (in number of spikes per trial) in VPm and S1 with a specific amount of jitter (zero-mean Gaussian noise of a specific standard deviation, sigma) as a function of thalamic synchrony. For a not-connected pair, the increase in thalamic synchrony and VPm and S1 firing were simulated by systematically adding spikes from another pair of neurons that is putatively connected. The assumption here 
514 was that a not-connected pair would become more identical to its neighboring neuron

515 (that has a functional connection to the downstream S1 neuron) with increasing

516 synchrony. This was repeated from 100-500 iterations for each synchrony level and the

517 probability of satisfying Criterion 1 and 2 was computed.

\section{Results}

Here, we present a comprehensive experimental and analytic framework for assessing synaptic connectivity using extra-cellular spiking activity from simultaneously recorded single units across brain structures. First, we provide a rigorous experimental protocol for performing simultaneous single-unit electrophysiological recording in topographically aligned regions in the thalamocortical circuit of the somatosensory pathway using a combination of stereotaxic targeting based on anatomical landmarks, sensory-evoked response properties, and post-hoc histological validation. We highlight some similarities and differences of the technical aspects of paired recordings performed in rats versus mice, two commonly used rodent species in mammalian electrophysiology. Next, we generate inferences regarding the synaptic connectivity of two single units based on spike correlation analysis across the presynaptic VPm and postsynaptic S1 spiking, leading to classification as either "connected" or "not connected", using an approach to be explained in detail below. Figure 1A highlights the basic experimental setup and analysis utilized in this study, with electrophysiological recordings targeted to primary somatosensory "barrel" cortex (S1, red) and to ventro-posteromedial thalamus (VPm, blue), during controlled deflections of a single whisker on the contralateral side of the face with a computercontrolled actuator (see Methods). In the context of a signal detection framework, we then quantify the effects of experimental data-length and local synchrony of presynaptic neurons on monosynaptic connection inference, and importantly, expand this framework to attach statistical levels of confidence to the synaptic connectivity inferences.

\section{Experimental workflow to establish paired recordings}

Paired recordings in topographically aligned feedforward sensory regions in-vivo have been previously shown to be experimentally tractable (Bruno and Sakmann 2006; Bruno and Simons 2002; Reid and Alonso 1995; Swadlow and Gusev 2001; Wang et al. 2010) yet it remains a challenging process, and to our knowledge, detailed reports on experimental approaches have not been published in full. Here, we documented the steps in details. Figure 1B summarizes the general workflow for establishing and analyzing paired recording: 1) mapping primary somatosensory cortex (S1), 2) localizing the ventral posteromedial (VPm) nucleus of the thalamus, 3) achieving and verifying topographical alignment of recording electrodes across VPm and S1,4) connectivity assessment 
552 through statistical analysis of the measured spiking activity from pairs of single-units across recording sites, and 5) histological verification of recording site locations.

Although the final electrode placement for paired recording involved thalamic electrode placement followed by placement of the cortical electrode, we found that an initial somatotopic mapping of cortex was critical for efficiently achieving topographical alignment. As part of the approach, we thus first performed coarse cortical mapping prior to thalamic localization. In rat, we employed primarily electrophysiological mapping approaches for this coarse mapping of S1. We targeted S1 using approximate stereotaxic coordinates and inserted a single electrode to obtain the functional location of several "barrels" using bregma as a reference point. Once we located the S1 region, we identified the stereotaxic location of three barrel columns containing neurons that were responsive to the movement of single primary whiskers (See Supplementary Figure 1 https://doi.org/10.6084/m9.figshare.14393528.v1). We used this relative distance between columns/barrels to estimate the overall topography of $S 1$ by overlaying a barrel map template scaled to fit the three data points. In mouse, taking advantage of the optical properties of the mouse skull (i.e. that it is relatively translucent), we employed intrinsic optical signal imaging (IOS) for the coarse cortical mapping. Intrinsic optical signals were acquired in response to separate, punctate deflections of three different single vibrissae. The corresponding cortical regions of activation were co-registered with the anatomy of the blood vessels and further used as a landmark for electrode placement. This triangulation methodology was adapted from previously published method from our laboratory (Gollnick et al. 2016; Millard and Stanley 2013). An example of this is shown in Figure 2A (see also Supplementary Figure 1A). The three images on the top row were acquired in response to punctate deflections of the Beta whisker (wBeta), the C2 whisker (wC2), and the B2 whisker (wB2), respectively. Each image represents the mean 0- to 6s post-stimulus response (baseline subtracted and scaled) to a 4-s $10-\mathrm{Hz} 1000 \mathrm{deg} / \mathrm{s}$ pulsatile stimulus, with the region of cortical activation appearing as a dark spot near the center of the image, at different locations for each whisker. We then fitted a barrel map template, recovered from histological brain sections from previous experiments onto the overall optical image of the brain surface through the thinned skull (template shown in bottom left of Figure 2A). The three centroids of the intrinsic imaging signals were used as reference points (Figure $2 \mathrm{~A}$, bottom middle) for transformation of the barrel map template involving scaling, rotation, translation and shearing. This transformed map along with the blood vessel image (Figure $2 \mathrm{~A}$, bottom right) was then used as a navigation guide for electrode placement for targeting a desired barrel column.

590

Once the cortex was coarsely mapped using the above approaches, we inserted an electrode to localize VPm (see Method: Electrophysiology). Specifically, we identified 
specific thalamic barreloids by manually deflecting each of the facial vibrissae individually in order to find an isolated unit that was maximally responsive to a single whisker only. Note that the procedure was nearly identical for rat and mouse, except that for the mouse, a silicon multi-electrode probe was utilized, and localization was performed using one of the probe sites, usually the deepest probe contact. Given that the probe was spanning several hundred microns on the thalamic recording site, we typically recorded from one to three barreloids simultaneously with two or more recording sites within a single barreloid. Final placement of the electrode was fixed when well-isolated units were detected on probe contacts. For verification of barreloid targeting within VPm, we compared local field potential (LFP) responses to individual stimulation of multiple nearby vibrissae. Figure 2B shows an example of LFPs recorded in the A3 barreloid of thalamus on one of the probe contacts. Note that the evoked LFP response exhibits an initial negative peak for the whisker A3 deflection (wA3, blue) that is substantially larger than responses to the deflection of other whiskers.

A practical challenge lies in the positioning of the recording electrodes to reach the multiple target areas. In a small subset of experiments, we optimized the electrode insertion angle by coating the electrodes with fluorescent dye (Dil) to mark the electrode track for post-experiment histological analysis. A range of insertion angles was tested for both S1 and VPm in mouse and rat. We found that the optimal electrode angle to target VPm thalamus in both rats and mice was 0 degrees from vertical (perpendicular to the brain surface) (Figure 2C left and Supplementary Figure 1B) For S1 targeting, we found that the optimal angle for rats was approximately 40-45 degrees from vertical and was approximately 30-35 degrees from vertical for mice for electrode penetration parallel to the cortical column/barrel, important for accurate targeting of specific cortical depths. The target recording site depths were determined based on published anatomical locations of the target structures (see Methods). Figure 2C shows typical electrode tracks targeting $\mathrm{S} 1$ and $\mathrm{VPm}$ in a mouse (left), along with the corresponding raw extracellular recordings from one VPm and one S1 electrode recording site (middle). Both the VPm and S1 extracellular recordings show sensory driven responses to deflections of the same whisker, the pattern of which is shown in the extracellular recordings of Figure $2 \mathrm{C}$. The times of whisker stimulation are denoted with the green arrows, representing a pattern of two isolated whisker deflections, followed by an $8 \mathrm{~Hz}$ train of whisker deflections. The raw electrophysiological recordings were subsequently sorted into single-unit data based on conventional spike sorting approaches and only well-isolated units were retained for analysis (see Methods). For each recording, the identified putative single unit spiking (red, blue) is superimposed on the raw recording (light gray), and the corresponding spike times are denoted above each trace (red and blue triangles). For each case, the spike waveform and spike auto-correlogram are shown to the right in Figure $2 \mathrm{C}$. A summary of 
630 the waveform size and isolation quality for all recorded VPm and S1 units is shown in 631 Supplementary Figure 1C.

\section{Sensory response in topographically aligned VPm - S1 layer 4 regions}

Achieving topographically aligned recordings across corresponding thalamic barreloids and cortical barrel columns necessitates the accurate targeting of the thalamic recording electrode/probe to the VPm nucleus of the thalamus. In particular, it is key to be able to distinguish whisker-responsive units located in VPm from those located in the adjacent posteromedial nucleus (POm) of the thalamus. In previous studies, POm units have been shown to exhibit lower evoked firing rate (Ahissar et al. 2000; Diamond et al. 1992; Landisman and Connors 2007; Sosnik et al. 2001) and much broader receptive fields than VPm units, responding to approximately six vibrissae on average, ranging from three to twelve whiskers (Castejon et al. 2016; Diamond et al. 1992). Hence, to ensure recordings from primarily VPm neurons, we selected thalamic units that showed restricted whisker sensitivity, usually only to one principal whisker (as previously shown in Figure 2B, VPm targeting (LFP)). The thalamic single units kept for subsequent connectivity analysis exhibited strong, reliable response (response reliability: $52.6 \pm 24.62 \%$, not shown) to a punctate whisker stimulus with short latency ( $1^{\text {st }}$ pulse (rats): $8.39 \pm 1.87 \mathrm{~ms}$ (mean \pm SEM), last pulse (rats): $12.3 \pm 3.04$ ms (mean \pm SEM), $n=24$ neurons, $N=12$ rats, $1^{\text {st }}$ pulse (mice): $9.37 \pm 1.39 \mathrm{~ms}$ (mean \pm SEM), last pulse (mice): $13.1 \pm 2.78 \mathrm{~ms}$ (mean $\pm \mathrm{SEM}$ ), $\mathrm{n}=39$ neurons, $\mathrm{N}=4$ mice, Figures $2 \mathrm{D}$ and $2 \mathrm{E}$, Supplementary Figure $\underline{2 A})$. As an additional criterion, we measured the adapting properties of the thalamic units in response to repetitive, periodic whisker stimuli (Ahissar et al. 2000; Masri et al. 2008) (Sitnikova and Raevskii 2010). Previous studies have shown that neurons in POm exhibit dramatic adaptation to persistent sensory stimulation, where most Pom neurons failed to exhibit a response to stimuli $\geq 11 \mathrm{~Hz}$ (Masri et al. 2008), and a significant shift in response latency with the adaptation, as compared to VPm neurons (Ahissar et al. 2000; Sosnik et al. 2001). Here, we showed a representative thalamic unit spiking response to an $8 \mathrm{~Hz}$ adapting whisker stimulus on the left in Figure 2D, with a PSTH in response to the full stimulus train (top) and a superposition of the stimulus evoked response to the $1^{\text {st }}$ (blue) 660 and $8^{\text {th }}$ (gray) deflection in the train (bottom). This particular example shows strong 661 stimulus-locked responses and moderate adaptation (i.e. reduction in response 662 amplitude), with a relatively small increase in latency of spiking. In fact, all the thalamic 663 units that we considered for further analysis showed only moderate spike frequency 664 adaptation, with a maximal increase in latency of $8 \mathrm{~ms}$ when comparing responses to the 665 first and last stimulus of the train (Figure 2D Right, mean first spike latency (FSL), latency 666 shift from Not-Adapted to Adapted $=4.28 \mathrm{~ms} \pm 2.32 \mathrm{~ms}$ (mean $\pm \mathrm{SEM}$ ), $\mathrm{n}=21$ cells from 667 rat; $3.71 \pm 3.11 \mathrm{~ms}$ (mean $\pm \mathrm{SEM}$ ), $\mathrm{n}=39$ neurons, $\mathrm{N}=4$ mice, Figure 2D right, inset). 668 Furthermore, the response latency of thalamic neurons measured here is consistent with 
669 the latency of VPm neurons (peak latency $<10 \mathrm{~ms}$ ) identified through a genetic validation

670 approach (Wright et al. 2021). Note that there remains a small possibility that a subset

671 of the thalamic units is POm in origin, and project to S1 inter-barrel areas (septa).

672 However, this is unlikely to be the case for the thalamocortical pairs that showed

673 monosynaptic connectivity, given that these thalamic and cortical units demonstrated

674 mostly single whisker receptive fields (Furuta et al. 2009) and the likelihood of detecting

675 synaptic connections in POm-S1 inter-barrel areas is much lower. Finally, a recent study

676 showed that POm cells remain largely inactive (close to zero spontaneous firing rate)

677 under isoflurane anesthesia (Zhang and Bruno 2019), making them extremely difficult to

678 locate and record from under the conditions of this study, which when combined with the

679 other factors described above make it unlikely that any of the recordings here are POm

680 in origin. Again, note that the challenges here are somewhat specific to this particular

681 brain region, but achieving definitive recordings in other brain regions would likely have

682 similar challenges to those described here.

683

684 Previous studies have shown that the convergence of thalamic inputs onto 685 topographically aligned cortical layer 4 neurons is generally relatively high, but with the 686 probability of contacting regular spiking units (RSUs) much lower than fast spiking units 687 (FSUs) (Bruno and Simons 2002; Swadlow 2003). We recorded from well-isolated cortical 688 layer 4 neurons (see Supplementary Figure 1C) at a cortical depth taken from 689 micromanipulator readings (Rat: $803.28 \pm 175.28$ um, mean \pm SEM, $n=21$, data not shown; 690 Mouse: 350-700 um, $n=11$, see Figure 5B). All cortical units included in this study were 691 putatively layer 4 neurons. The mean first spike latency of cortical units in rat was 11.4 $692 \mathrm{~ms} \pm 1.77 \mathrm{~ms}$ (mean $\pm \mathrm{SEM}, \mathrm{n}=22$ ) and $12.6 \pm 2.06 \mathrm{~ms}$ (mean $\pm \mathrm{SEM}, \mathrm{n}=11$ ) for mouse. 693 We found that the relative latency difference between VPm and S1 layer 4 in aligned 694 regions was comparable to the expected synaptic delay between VPm and S1 layer 4 695 (differences between VPm-S1 were $~ 3 \mathrm{~ms}$ for both rat and mouse). An example of the 696 response to a punctate whisker stimulus for a pair of VPm and S1 FS units with mean 697 first-spike latency of $7.5 \mathrm{~ms}$ and $11.2 \mathrm{~ms}$ is shown on the left of Figure $2 \mathrm{E}$.

\section{Inferring connectivity in the context of a signal detection framework}

701 In general, neurons in the central nervous system require the concerted action of a 702 relatively large number of pre-synaptic inputs to produce an action potential (Bruno and 703 Sakmann 2006). Thus, the relationship between pre- and post-synaptic neurons is 704 tenuous at best, reflected in often a very subtle increase in the probability of firing of the 705 post-synaptic neuron a few milliseconds after the firing of a pre-synaptic neuron. The 706 analysis of spike trains from a pair of neurons can thus be utilized for a simple binary 707 classification of a neuronal pair either being 'not connected' or 'connected' (Figure 3A). 
708 In order to infer connectivity, for each recorded pair of neurons, the raw spike cross-

709 correlogram is calculated using standard approaches ( $\mathrm{C}$, Figure 3C) (see Methods),

710 effectively yielding a histogram of cortical firing relative to thalamic spike times. Because

711 the analysis is based on spiking activity, and the baseline spiking activity can often be

712 relatively low, we drove the thalamocortical circuit in-vivo with a weak whisker stimulus

713 (Figure 3B, sinusoidal deflection, $4 \mathrm{~Hz}$, mean velocity: $25 \%$ s), which is known to enhance

714 firing rates with minimal impact on firing synchrony across neurons (Bruno and Sakmann

715 2006). To correct for correlated stimulus-locked activity, we generated a shuffled-

716 corrected spike cross-correlogram ( $\mathrm{C}_{\mathrm{sc}}$, Figure $\left.3 \mathrm{E}\right)$ by subtracting the trial shuffled spike

717 cross-correlogram (Cs, Figure 3D) from the raw spike cross-correlogram. The "shuffled

718 cross-correlogram" is generated using the same procedure used for the raw cross-

719 correlogram, except that the trials of the thalamic and cortical spiking activity are

720 randomized relative to each other. This effectively destroys any elements of the cross-

721 correlogram that are not due to the stimulus. Shown in this example is the qualitative

722 signature of monosynaptic connectivity - a prominent peak in the shuffled-corrected

723 cross-correlogram for small positive lags that would be consistent with a single synaptic

724 delay.

725

726 Then, to conclude that a neuronal pair was 'connected', we adopted two criteria that 727 expanded from previous studies (Bruno and Sakmann 2006; Reid and Alonso 1995;

728 Swadlow and Gusev 2001) based on both the raw and shuffled-corrected cross729 correlogram: (Criterion 1) a notable sharp, millisecond-fine peak is observed within a

730 narrow lag of 1-4 ms after a thalamic spike, and (Criterion 2) this fast 'monosynaptic peak'

731 is significant or still present after accounting for (subtracting) stimulus-induced correlation.

732 Note that a peak is defined as the bin in the raw cross-correlogram ( $0.5 \mathrm{~ms}$ bin size) that

733 contains the maximum number of events. In order to fulfill both criteria, we required that

734 the peak detected in the 1-4 ms range to have the largest correlation out of all the bins in

735 the range of $+/-25 \mathrm{~ms}$ window and this correlation is significant (>3.5 SD) against shuffled

736 data. For the example 'connected' pair in Figures 3C-E and a separate 'not connected'

737 pair, Criterion 1 is evaluated from the raw cross-correlation as shown in Figure 4A. For

738 the 'not connected' example (raw cross-correlogram on the left), a peak was detected

739 outside the central $1-4 \mathrm{~ms}$ lag, and thus fails Criterion 1. The example 'connected' pair,

740 the raw cross-correlogram on the right, exhibits a peak within the central 1-4 ms lag

741 (shown as a vertical gray band), and thus passes Criterion 1. Figure 4A illustrates how

742 Criterion 2 is estimated from the shuffled-corrected cross-correlation. We evaluate the

743 prominence of the peak relative to a distribution of peak magnitudes (same bin with

744 maximum number of events) from the shuffled cross-correlograms (1000 iterations).

745 Specifically, we define the metric related to Criterion 2 as the peak height, $\mathrm{h}$, computed

746 using the maximum of the $\mathrm{C}_{\mathrm{sc}}(0.5 \mathrm{~ms}$ bin size) within $1-4 \mathrm{~ms}$ lags, normalized by the 
standard deviation of the shuffled cross-correlogram. This metric is thus the number of standard deviations the peak of the shuffled-corrected cross-correlogram within the 1$4 \mathrm{~ms}$ lag is above the shuffled distribution. This is shown in more detail for this example in the inset in the bottom row of Figure 4A, where the central portions of the shuffledcorrected cross-correlograms are shown for each case. In order to pass Criterion 2, the peak height of the shuffled-corrected cross-correlograms must be greater than 3.5 standard deviations of the shuffled data, which corresponds to a $99.9 \%$ confidence interval for each bin.

Thus, we conclude that the shuffled-corrected cross-correlograms on the left fails Criterion 2 as the peak falls below the criterion line (3.5 SD, depicted as dashed line) whereas the shuffled-corrected cross-correlograms on the right passes Criterion 2. Note that for the "not connected" pair shown in Figure 4A, the shuffled-corrected crosscorrelogram also revealed the presence of the global peak outside the 1-4 ms central lag, similar to that of the raw cross-correlogram. Thus, it is possible to utilize the shuffledcorrected cross-correlogram for the evaluation of Criterion 1 in some cases, but overall, we found that Criterion 1 was more robustly evaluated utilizing the raw cross-correlogram. The raw and shuffled-corrected correlograms for all recorded thalamocortical pairs are shown in Figure 4B - normalized to the peak in each correlogram, ordered from earliest to latest peak, top to bottom. The vertical lines in these plots highlight the $1-4 \mathrm{~ms}$ lag range described in Figure 4. Figure 4C shows the distribution of the lags of the peak location in the raw and shuffled-corrected correlograms across all recorded pairs. Overall, the peak locations for raw and shuffled-corrected correlograms were similar, shifted only by 1-2 bin size $(0.5 \mathrm{~ms})$. For all the monosynaptically connected pairs in this paper, the mean peak location was $1.18 \pm 1.08 \mathrm{~ms}$ (mean \pm SEM, $\mathrm{n}=11$ ), and with a median of $1.5 \pm 0.89 \mathrm{~ms}$.

Note that to be classified as connected, a candidate pair of neurons must pass both

774 Criterion 1 and Criterion 2. Using these metrics and criteria, we can consider the result of cross-correlation analysis for each pair as an inference problem in the context of a signal detection framework, where we conceptualize the distribution of metric values for the non-connected pairs as "noise", and for the connected pairs, as "signal". Applying the criteria on the metric values yields four possible outcomes: Hit (connected pair classified/inferred as such), Miss (connected pair classified/inferred as 'not connected'), False Alarm (not-connected pair classified/inferred as 'connected'), and Correct Reject (not-connected pair classified/inferred as such) (Figure 4D). With these metrics and criteria, we classified measured neuronal pairs into 'connected' and 'not-connected'

784 distributions, with 11/42 pairs having a putative monosynaptic connection and 31/42 pairs having no apparent connection (Figure 4D bottom, Rat: $\mathrm{n}=22$ pairs, Mouse $=20$ pairs). For Criterion 1, this was a binary classification due to biological constraints (single 
synaptic delay) - pairs having peak locations from their raw cross-correlograms within the $1-4 \mathrm{~ms}$ lag pass Criterion 1, and those which do not, fail Criterion 1. For Criterion 2, the dashed vertical line represents the 3.5 SD for the peak height metric - pairs having peak height above this criterion line pass Criterion 2. Only pairs that passed both Criterion 1 and 2 in Figure 4D (bottom) were classified as 'connected' (anything to the right of vertical dashed line and on the top row) and the rest were classified as 'not connected'. Note that while this is a binary classification, and the end-result is a labeling of a pair as 'connected' or 'not-connected', not all classifications are equivalent, with some having substantially more confidence in the classification than others. By utilizing a bootstrapping approach (see Methods) and evaluating the likelihood of specific outcomes using the pre-defined metrics and criteria for each recorded pair, we were able to attach a probability measure (Figure 4E bottom, color bar, $\mathrm{P}($ Inferring 'connected')) along with each connectivity inference. In Figure 4E bottom, some of the cases that passed Criterion 1 have peak heights that are substantially far away from the line for Criterion 2. As expected, the likelihood of inferring monosynaptic connection for these cases was higher ( 0.6-1) and thus we have more confidence in the assertion, as compared to cases that are very close to the criterion line for Criterion 2, or failed Criterion 1.

Probabilistic measure of connectivity inference across brain structures in largescale recordings

Through the advent of high channel-count electrophysiological recording techniques (Chung et al. 2019; Jun et al. 2017; Rios et al. 2016), the diversity of recording quality, cell type, and possibilities for connectivity has expanded tremendously. To demonstrate how our framework can be beneficial, Figure 5 shows an example of simultaneous topographically aligned recordings from silicon multi-electrode probes inserted in VPm thalamus (Figure 5A) and S1 (Figure 5B). Specifically, focusing on portions of the probes that were assessed to be topographically aligned, a subset of channels from the VPm probe (Channels A-D) yielded 5 thalamic neurons (VPm 1-5), while a subset of channels from the $S 1$ probe $(A-D)$ yielded 4 cortical neurons (S1 1-4). These neurons were recorded during weak sinusoidal $(25 \mathrm{deg} / \mathrm{s}, 4 \mathrm{~Hz})$ whisker stimulation. In this multidimensional analysis, each of the 20 thalamocortical pairs were assessed for the possibility of connectivity. Table 1 in Figure $5 \mathrm{C}$ shows the matrix of binary outcomes of monosynaptic connectivity inference from cross-correlation analysis of the full dataset across the recording sites. Out of the $20 \mathrm{VPm}-\mathrm{S} 1$ pairs, 4 were judged to be connected according to our criteria. Note that while the connectivity from an individual barreloid to the homologous cortical barrel is relatively high (it has been estimated that approximately 1 in 3 VPm neurons within a barreloid is connected to a particular neuron in cortical layer 4 of the homologous barrel (Bruno and Sakmann 2006)), the relatively stringent criteria 
824 of the inference coupled with other factors such as the potential to record from multiple 825 nearby VPm barreloids and variable single unit quality make this outcome typical. Shown 826 in Figure 5C Table 2 are the probabilities of connectivity associated with each pair using 827 the bootstrapping method ( $n=1000$ iterations) on the data (Table 2), as previously 828 described. In general, the binary inference for a particular pair corresponded to the 829 bootstrapped confidence levels - connected pairs had a relatively high probability of 830 connectivity from the bootstrapping (see all 'connected' pairs in Supplementary Figure 4) 831 while not connected pairs had a relatively low probability of connectivity from the 832 bootstrapping $(p<0.4)$. We found that the binary outcomes of monosynaptic connectivity 833 inference could result in different ranges of probability of inferring monosynaptic 834 connection (0.4-1). One factor that could affect this probability is data-length. Not 835 surprisingly, we found that the 'connected' pair that yielded the lowest probability in this 836 matrix ( $p=0.41$ ) had about 3900 spikes (in terms of geometric mean), as compared to 837 the others $(4900,6900$ and 10000 spikes).

\section{Data-length effect on monosynaptic connection inference}

841 As previously shown, inference of monosynaptic connectivity using cross-correlation 842 analysis on extracellular signals is highly dependent on the amount of collected data. 843 Based on existing literature, the recommended numbers of spikes were highly variable, 844 ranging from 2000 to 10000 spikes (Swadlow and Gusev 2001; Wang et al. 2010), but 845 were presented more as a "rule-of-thumb" than based on systematic evaluation. Here, we 846 systematically evaluated data-length dependence effects on the connectivity inference 847 outcomes. More specifically, we measured the data-length effect by performing 848 bootstrapping on the full dataset by randomly selecting segments of data of increasing 849 duration, as illustrated in Figure 6A. Note that for this analysis, the effects of data-length 850 were evaluated in the context of Criterion 2, as this is the measure more prominently 851 affected by data-length. In general, the analysis is primarily sensitive to the number of 852 spikes used in the estimates, rather than the time duration of experimental data collection, 853 and is sensitive to the number of both VPm and S1 spikes (i.e. sufficient spiking from both 854 is requisite). For this reason, we utilized the geometric mean of the number of VPm and 855 S1 spikes, calculated as the square root of the product of the number of VPm and S1 856 spikes. Because we did not have access to an established "ground truth" of a pair being 857 either connected or not connected, we utilized example pairs in which the analysis 858 revealed a very clear classification, which we subsequently utilized as ground truth for the 859 analysis.

861 Shown are the results from such an analysis for a 'not connected' pair (Figure 6B-E) and 862 a 'connected' pair (Figure 6F-I). We found that, when a functional connection was 
863 obviously not present, the correlated firing activity between a pair of neurons was 864 indistinguishable from that expected from stimulus-induced correlation, reflected in the 865 metric remaining well below the criterion line (3.5 SD) for all values of data-length, while 866 however steadily increasing as a function of the geometric mean (also accompanied by 867 a decrease in the variability of the metric as reflected in the SEM) (Figure 6C). Figure 6D 868 shows the distributions of the estimated connectivity metric $h$ for two particular data869 lengths (geometric means of 1837 spikes and 4730 spikes), relative to the criterion line. 870 Both of these distributions are clearly to the left of the criterion line, corresponding to high 871 correct reject and low false alarm rates, relatively unaffected by data-length (Figure 6E).

873 In contrast, when a functional connection was apparent, limited data confounded the 874 inference of 'connected' as the metric remained below the criterion line for smaller data875 lengths (Figure 6G). For two particular data-lengths (geometric means of 3760 and 10201 876 spikes), the distributions of the estimated connectivity metric $\mathrm{h}$ are shown in Figure $6 \mathrm{H}$, 877 relative to the criterion line. While the longer data-length resulted in a distribution of the 878 peak height that was clearly above the criterion line (easily passing Criterion 2), the 879 shorter data-length resulted in a distribution whose mean was above the criterion line 880 (barely passing Criterion 2), but with a substantial portion of the distribution below the 881 criterion line, resulting in a hit rate of $87.7 \%$ and miss rate of $12.3 \%$. We found that the 882 data-length required to reach a consistently correct inference was approximately 5000 883 spikes for this example (Figure 6I).

From the results in Figures 6B-E and 6F-I, it appears that data-length was critical only for correctly identifying a connected pair, and did not directly affect the inference related to a "not connected' pair - in other words, the analysis is prone to type II errors as opposed

888 to type I, and the type II error is strongly dependent upon data-length. It is important to 889 point out that the data-length needed for sufficiently reducing the probability of error in the 890 inference in Figure 6l is dependent upon the ground truth value for the connectivity metric $891 \mathrm{~h}$, as well as the variance in the estimator. We thus analyzed the estimator bias and 892 variance across six 'connected' pairs, as a function of the data-length, which we could 893 systematically vary by utilizing sub-sampling of the full datasets. Figure 6J shows the bias 894 in the estimator for the connectivity metric $h$, as a function of the geometric mean number 895 of spikes, with generally a negative bias (an underestimate) and a clear exponential 896 decrease in bias with data-length as expected, as the full data-length is approached. 897 Figure 6K shows the corresponding variance in the estimator of the connectivity metric $h$, 898 again as a function of the geometric mean number of spikes. Although some of the 899 measures exhibited an apparent invariance to the data-length, overall, this quantity 900 decreased exponentially with increasing geometric mean number of spikes, again as 901 expected. For the set of 'connected' pairs of thalamic and cortical neurons here, the 
902 geometric mean number of spikes required for the lower bound of resampled data to pass

903 Criterion 2 is shown in Figure 6L, with a median of approximately 9000 spikes.

\section{Thalamic synchrony effects on monosynaptic connection inference}

Without having access to the subthreshold activity of the postsynaptic cortical neurons, assessment of connectivity through the co-occurrences of spiking activity in thalamic and cortical neurons often necessitates activation of the intact circuitry with exogenous stimulation due to relatively low intrinsic spontaneous firing rates. The analysis of Figure 6 revealed a clear motivation for acquiring larger numbers of spikes, which can obviously be facilitated through increased mean firing rates. In sensory pathways, previous studies have used sensory stimuli of varying strengths to evoke higher firing rate in primary sensory areas (Bruno and Sakmann 2006; Reid and Alonso 1995; Sedigh-Sarvestani et al. 2017; Wang et al. 2010), citing a rule-of-thumb which involves increasing the number of spikes through external stimulation, but not with a stimulus so strong as to induce stimulus-driven synchronization in spiking. Although this approach is logical, it remains ad hoc, and the exact ramifications are not clear. Here, we utilized silicon multi-electrode probes in the thalamic VPm and S1 layer 4 to quantify changes in neural activity across spontaneous and stimulus driven conditions, and performed analyses to systematically evaluate the potential effects of stimulus-driven changes in firing rate and synchrony on the monosynaptic connection inference between VPm and S1 pairs. Figure 7A shows simultaneous recordings from two thalamic neurons (VPm1 and VPm2) within the same thalamic barreloid and two corresponding cortical S1 neurons in the homologous barrel column. For this example, VPm Unit 1 and S1 Unit 1 were revealed to be 'connected', while VPm Unit 2 and S1 Unit 2 were clearly 'not connected'. Under increasingly stronger stimulus drive, from spontaneous (left), to weak-sinusoidal whisker stimulus drive (middle), and to strong repetitive, punctate whisker stimulus drive (right), there is a general increase in firing rate across the recorded cells in both VPm and S1 (Figure 7B). In addition to the modulation of mean firing rate, we found that increasing stimulus drive affected the inference of connectivity by producing inconsistent conclusions across stimulus conditions, especially obvious with increasingly strong stimuli. Interestingly, we found that this preferentially affected the location of the peak in the cross-correlogram, which is associated with Criterion 1 evaluated through the raw cross-correlogram. Figure 7C shows the raw and shuffled-corrected spike cross-correlograms across the stimulus conditions for the connected pair VPm Unit 1-S1 Unit 1, and Figure 7D shows this for the not-connected pair VPm Unit 2-S1 Unit 2. Note that we were able to maintain stable recordings for this particular group of neurons over a relatively long experimental time period, enabling us to collect a sufficient number of spikes in the spontaneous condition

940 here (VPm Unit 1-S1 Unit 1 connected, VPm Unit 2-S1 Unit 2 not-connected). First 
941 considering the connected pair VPm Unit 1-S1 Unit 1 (Figure 7C), we found that the

942 inference remained consistent ('connected') for spontaneous (I) and sinusoidal (II)

943 stimulus conditions as it passed both Criterion 1 and 2. However, for the repetitive

944 transient (III) stimulus condition (bottom row), while the peak height in the shuffled-

945 corrected cross-correlogram exceeded the criterion line (thus satisfying Criterion 2, 7C

946 bottom panel), there was a disappearance of an isolated peak in the 1-4 ms lag of the

947 raw cross-correlogram, which is a violation of Criterion 1. This led to the incorrect

948 inference of 'not connected' for the case of the strong, transient stimulus, or a "miss" in

949 the language of signal detection theory. In our analysis, we have 12 thalamocortical pairs

950 for which we have all stimulus conditions, where we consider the spontaneous condition

951 as the "ground truth". Of these 12 pairs, 3 pairs were connected (C) and 9 not connected

952 (NC), as determined from the spontaneous condition, which we consider "ground truth".

953 For the 9 NC pairs, 6 pairs were incorrectly classified as C in the strong (transient)

954 stimulus case, and the remaining 3 pairs continued to be correctly classified as NC. For

955 the $3 \mathrm{C}$ pairs, 2 pairs were incorrectly classified as NC in the strong (transient) stimulus

956 case, and the remaining 1 pair continued to be correctly classified as $C$. For this relatively

957 small number of pairs, it is difficult to identify a particular pattern in this, but we can

958 generally say that both types of errors can emerge from the strong stimulus that

959 synchronizes the thalamic population, as we also demonstrate in the simulations.

961 Turning to the 'not-connected' pair VPm Unit 2-S1 Unit 2 in Figure 7D, note that for the 962 spontaneous (I) and sinusoidal (II) case, while the peak height of the shuffled-corrected

963 cross-correlogram exceeded 3.5 SD (thus passing Criterion 2, not shown), we correctly 964 inferred 'not connected' for these stimulus conditions given the violation of Criterion 1. 965 Surprisingly, we incorrectly inferred 'connected' for the repetitive transient (III) stimulus 966 condition as fast peaks emerged in the 1-4 ms lag of the raw cross-correlograms, 967 satisfying Criterion 1 (and also Criterion 2, not shown), or a "false alarm" in the language 968 of signal detection theory. We thus encountered two kinds of errors when utilizing 969 exogeneous stimulation to increase neuronal firing rates - "misses" and "false alarms", 970 both linked to Criterion 1, due to appearance of spurious peaks in 1-4 ms lag in the cross971 correlogram.

972

973 In previous studies, increase in stimulus-driven firing rate has been shown to couple with 974 higher degree of synchronization within and across neural circuits (Temereanca et al. 975 2008; Wang et al. 2010) that can potentially dominate the temporal relationship of the 976 network dynamics revealed by correlational analysis (Ginzburg and Sompolinsky 1994). 977 This highlights the issues underlying the tradeoff between firing rate and synchronization 978 of the local network that are intrinsic to the correlational analysis used for monosynaptic 979 connection inference. Several paired recording studies have approached this problem by 
980 collecting spontaneous spiking data (Swadlow and Gusev 2001), where presumably the 981 spontaneous activity is less synchronous than stimulus driven activity, or by providing 982 weak or non-synchronizing inputs to probe the circuit (Bruno and Sakmann 2006; Bruno 983 and Simons 2002; Reid and Alonso 1995). However, some degree of synchronization is 984 always present, and a direct understanding of the potential effects and implications of 985 population synchronization of presynaptic neurons on monosynaptic connection 986 inference is lacking. To relate the amount of synchronization across the thalamic units 987 with the increase in stimulus strength, we quantified synchrony using the spike cross988 correlogram measured across thalamic pairs. Specifically, the synchrony was defined as 989 the number of spikes of the cross-correlogram within a window of $+/-7.5 \mathrm{~ms}$, normalized 990 by the number of thalamic spikes (see Methods). Population data showed significant 991 increases in thalamic synchrony comparing spontaneous and transient conditions, as well 992 as sinusoidal and transient conditions (Figure 7E, $\mathrm{n}=15$ pairs). The raw spike cross993 correlograms for the two VPm units shown in the top row of Figure 7A are shown in Figure $9947 \mathrm{~F}$ (top and orange symbol in bottom), illustrating an increase in the central peak of the 995 cross-correlogram and thus the thalamic synchrony, note the emergence of sharp peaks 996 from spontaneous to transient stimulus. The nature of the stimulus thus strongly affects 997 the synchrony, but also strongly affects the firing rate, suggesting a more general 998 relationship between firing rate and synchrony. To more generally quantify the 999 relationship between thalamic synchrony and mean firing rates, across several

1000 experiments we collected thalamic responses to different stimuli (spontaneous, sinusoidal 1001 (25 deg/s) and transient stimuli of different velocities (50, 125, 300, 600, 900, and 1200 $1002 \mathrm{deg} / \mathrm{s})$, producing a range of firing rates and corresponding degrees of synchrony, 1003 displayed in Figure 7F. In general, there was a monotonic increase in thalamic synchrony with firing rate, fit well by an exponential function (Figure 7F, blue curve).

1005

1006

To more systematically explore the relationship between the measured thalamic 1007 synchrony and Criterion 1 of the thalamocortical connectivity inference, we generated a 1008 set of simulations based on experimental data to demonstrate how increasing degrees of 1009 thalamic synchrony could influence the connectivity inference. Described in more detail 1010 below, the simulations were based on introducing spiking activity that corresponds to 1011 varying degrees of synchrony into the experimentally observed spiking activity at the 1012 levels of both thalamus and cortex. As described above for experimental observations, 1013 through these simulations we also found that the synchronization of the presynaptic 1014 thalamic population could potentially produce errors in two scenarios: misses (i.e. a 1015 connected pair being incorrectly inferred as "not connected", denoted $\mathrm{C} \rightarrow \mathrm{NC}$ ) and false 1016 alarms (i.e. a not connected pair being incorrectly inferred as 'connected', denoted NC $\rightarrow$ 1017 C).

1018 
1019 We first sought to simulate the scenario of the top row of Figure 7C where a pair of 1020 connected thalamocortical neurons (VPm Unit 1-S1 Unit 1) was misclassified as not-

1021

1022

1023

1024

1025

1026

1027

1028

1029

1030

1031

1032

1033

1034

1035

1036

1037

1038

1039

1040

1041

1042

1043

1044

1045

1046

1047

1048

1049

1050

1051

1052

1053

1054

1055

1056

1057 connected because a significant maximum peak was not detected in the central 1-4 ms lag of the raw cross-correlogram (violating Criterion 1, rightmost panel). We hypothesized that this experimental observation was due to a significant increase in cortical firing that was caused by inputs of nearby VPm neurons also connected to the same cortical neuron $\mathrm{S1}$, but relatively synchronous with the reference thalamic neuron VPm1. For this simulation, we therefore emulated this scenario by reintroducing jittered spikes from VPm Unit 1 and S1 Unit 1 back into these datasets, respectively. Importantly, we found from our experimental observations that the mean thalamic and cortical firing rate increased with stimulus strength, but cortical firing increased to a lesser extent as compared to thalamic firing (not shown). Therefore, the number of added spikes and the jitter (zeromean Gaussian noise of a specific standard deviation, $\sigma$ ) were both set to produce a specified level of synchrony between the original and perturbed VPm1 spike train and also match the observed firing rates in both VPm and S1 for this particular condition. The resultant thalamic and cortical activity was denoted as VPm Unit $1^{*}$ and S1 Unit 1*, respectively. A schematic of this was shown in Figure 8A. With these manipulations, we performed the analysis of connectivity as before between VPm Unit $1^{*}$ and S1 Unit $1^{*}$ at each firing rate level (with corresponding thalamic synchrony level) to generate the probability of the connectivity inference (i.e. probability of satisfying Criterion 1 reported as the fraction of bootstrapped iterations results in satisfying Criterion 1). We found that the probability of inferring a connection dropped with increasing thalamic synchrony as shown in the top of Figure 8B. For low levels of synchrony (e.g. thalamic synchrony measures of 0.2 or less), the probability of satisfying Criterion 1 (and thus making the correct inference, given that Criterion 2 is satisfied) remained relatively high (from $\sim 0.6$ to 0.9). However, increasing levels of thalamic synchrony (and certainly above 0.5), the probability of satisfying Criterion 1 approached 0 (and thus the chance of a miss neared $100 \%$ ). The raw spike cross-correlogram for two synchrony levels (highlighted with the green and blue vertical dashed lines in Figure 8B top) are shown below, illustrating the disappearance of the peak in the $1-4 \mathrm{~ms}$ lag with increased synchrony. This is consistent with what we observed in the actual experimental data (Figure $7 \mathrm{C}$, top right). Note that for artificially high levels of synchrony, approaching near perfect synchrony, the probability of satisfying Criterion 1 does gradually come back up and approach the same probability as for very low levels of synchrony, as expected (not shown for simplicity).

On the other hand, we found that in addition to causing the incorrect classification of a connected pair (i.e. a "miss"), the thalamic synchrony induced by increased stimulus drive could also have the opposite effect - the incorrect classification of a not-connected pair as connected (i.e. a "false-alarm). When considering a thalamocortical pair that is not 
1058 synaptically connected (VPm Unit 2-S1 Unit 2), increased stimulus drive introduces the 1059 presence of spiking in the S1 neuron induced by nearby VPm neurons that serve as 1060 synaptic inputs to the $S 1$ neuron in question. When these VPm neurons become 1061 increasingly synchronous with VPm Unit 2, this results in the presence of a peak in the 1062 raw cross-correlogram in the 1-4ms bin, satisfying Criterion 1 and thus resulting in a "false 1063 alarm", displayed in Figure 7E.

For a demonstration of this phenomenon, from experimentally obtained data, we conducted a simple simulation designed to systematically explore this effect. Specifically, in addition to the not-connected pair in question, VPm Unit 2-S1 Unit 2, we identified a distinct, simultaneously recorded thalamocortical pair that was inferred to be connected. By introducing spikes from the connected thalamic and cortical neuron into the spike trains of VPm Unit 2 and S1 Unit 2, respectively, the degree of thalamic synchrony was systematically increased as a function of the number of spikes added, but at the same time introduced the presence of cortical spiking from the connected pair. As with the first simulation, the number of spikes added to VPm Unit 2 and S1 Unit 2 was set to match the observed thalamic and cortical firing rates for this condition, to produce the perturbed spikes trains VPm Unit $2^{*}$ and S1 Unit $2^{*}$. A schematic of this was shown in Figure 8C. With these manipulations, we performed the analysis of connectivity as before, but now between VPm Unit $2^{*}$ and S1 Unit $2^{*}$ at each firing level (with corresponding thalamic synchrony level) to generate the probability of the connectivity inference (i.e. probability of satisfying Criterion 1 reported as the fraction of bootstrapped iterations results in satisfying Criterion 1), as with the first simulation. We found that as thalamic firing became more similar with increasing amount of mixing (reflected in an increase in thalamic synchrony), the raw cross-correlogram exhibited the increased likelihood of a peak in the $1-4 \mathrm{~ms}$ bin. Correspondingly, the probability of error rapidly increased with thalamic synchrony (probability of satisfying Criterion 1 exceeds 0.5 as thalamic synchrony reaches 1.5 in the top panel of Figure $8 \mathrm{D}$ ). The raw spike cross-correlogram for two synchrony levels (highlighted with the green and blue vertical dashed lines in Figure 8B 1087 right top), illustrated the appearance of the peak in the 1-4ms lag with increased 1088 synchrony (Figure 8D bottom right). Although these simulations should be considered 1089 primarily in terms of the basic trends they exhibit, the rapid increase in the probability of 1090 error with thalamic synchrony suggests that above some level of synchrony in the pre1091 synaptic population, false-alarms are inevitable. 


\section{Discussion}

1095 The field of neuroscience is in a period of rapid tool development, spawned by a combination of innovative technologies and a shift in the focus of international scientific priorities. The explosion of tools for performing large scale neuronal recording at single cell resolution provides access to real-time monitoring of network activity within and across multiple brain regions (Ahrens et al. 2013; Chung et al. 2019; Jun et al. 2017). This access enables the exciting potential for interpretation of the causal flow of neuronal activity that ultimately underlies brain function, shaping of perception, and behavior (Sheikhattar et al. 2018). There have been significant efforts in anatomical tracing of connectivity and cell-type specific projections within and across brain regions and hemispheres, leading to new insights into the detailed structure of brain circuits (Yuan et al. 2015). The ultimate goal is to understand how this complex structure gives rise to behaviorally relevant function through the dynamic interaction of neurons within the neural network. However, the identification of functional connections amidst perturbationinduced confounding variables in an intact brain remains very challenging despite increasing accessibility (Lepperød et al. 2018). Here, we provided an experimental and analytical framework for quantifying long-range synaptic connectivity that was developed and tested in the thalamocortical circuit of the rodent somatosensory pathway but is generalizable to other circuits and pathways. Importantly, we attacked this from a scalable statistical framework based on signal detection theory, where we established approaches to assess confidence in classification, and systematically examined factors contributing to the inference of connectivity.

The gold standard of studying and assessing synaptic connectivity involves direct manipulation of pre-synaptic neurons to observe a measurable postsynaptic effect. Hence, connectivity studies are often performed using in-vitro brain slices (Jiang et al. 2015; Pfeffer et al. 2013) due to better accessibility to pre- and postsynaptic neurons concurrently, which is difficult or intractable in-vivo (Jouhanneau et al. 2015; Jouhanneau et al. 2018; Pala and Petersen 2015). In attempts to identify connectivity in-vivo, previous studies have used electrical stimulation to conduct collision tests that involve comparing 1124 the timing of anti-dromic activation with stimulus evoked activity to verify origins of 1125 projection (Kathleen Kelly 2001; Swadlow 1989). Although this is a powerful and 1126 attractive approach, as it helps to more confidently assess connectivity and establish 1127 causal relationships, it does not scale well to assessing connectivity at the population 1128 level, where selective stimulation of individual neurons is not typically possible. As an 1129 alternative, we adopted non-synchronizing, weak sensory drive (Bruno and Sakmann 1130 2006; Wang et al. 2010) to assess likely connectivity through spike cross-correlation 1131 analysis. Although this approach obviously does not address issues of causality directly, 1132 it scales with increasing size of population recordings in the pre- and post-synaptic 
1133 regions, opening up the possibility for assessing connectivity using large, ensemble 1134 recordings (Juavinett et al. 2018).

The systematic evaluation of the parameters of analysis presented here offers the potential for the optimization of experiment design for the purpose of assessing connectivity. Although the details likely vary across brain region and experimental preparation, the results here do suggest some general rules of thumb, perhaps the most important of which revolve around the interplay between data-length and synchrony. For example, based on our data, we estimated that about 9000 spikes (in terms of geometric means of total thalamic and cortical spikes) were needed to reach an inference with high certainty (95\% confidence interval) in the fentanyl-anesthetized rat, consistent with recommended number of spikes used using similar stimulus conditions (Bruno and Simons 2002; Wang et al. 2010) and between 6800 and 10,000 spikes for spontaneous and sinusoidal conditions respectively in the isoflurane-anesthetized mouse (data not shown). Thus, the requirements were on the same order of magnitude for both of these cases, despite significant differences in experimental preparation (i.e. anesthesia, etc.) and differences in precise anatomical details across the species.

Synchrony across the pre-synaptic population has long been implicated as a potential confound in assessing synaptic connectivity, typically tied to false alarms (type 1 error), or the incorrect inference of connectivity in non-connected pairs (Ostojic et al. 2009). The results here supported this long-held assumption, where the artificially elevated synchrony in the VPm population resulted in the increased likelihood of satisfying Criterion 1, and importantly established ranges of measured synchrony for which this is more likely. Surprisingly, we also found that increased pre-synaptic synchrony could result in a different type of confound - a miss (type 2 error), or the incorrect inference of not-connected for connected pairs. Interestingly, this effect emerged over a relatively similar level of synchrony as determined through artificial elevation of the thalamic synchrony. As expected, spontaneous activity is typically fairly asynchronous, and in this regime, the types of errors described here are unlikely for this case. However, common drive through sensory input designed to elevate firing in otherwise sparse conditions increases the likelihood of these errors, and thus the tradeoff between increased data length and synchronization plays an important role in experimental design. This is further important in extending this approach to awake, behaving conditions, with increased firing rates, and continuous modulation in population synchrony by brain state. One alternate explanation for increased likelihood of synaptic connectivity classification errors as we moved from stimulus-free condition to highly synchronizing, transient stimuli could be due a higher rate of spike sorting errors, where elevated firing across all units (single-unit and multi-unit) could cause waveform distortion and hence cluster misclassification. Despite 
1172 a slight reduction in waveform signal-to-noise ratio (4\% change for VPm and $1.5 \%$ change 1173 for S1), however, we found no significant difference when we compared the waveform 1174 features of all single units such as peak-to-peak amplitudes and signal-to-noise ratios in 1175 spontaneous and stimulus-present conditions (see Supplementary Figure 6, mice data).

Although the approach here was developed with generality in mind, there are several potential limitations related to the specific details of the experimental preparation. First, the data collected in this study were from immobilized, anesthetized rodents. This enabled relatively stable and long-duration recordings of well-controlled stimulus conditions that provided insights for accurate identification of synaptic connectivity across different stimulus regimes. Although we envision the broad applicability of this approach for awake, paired recordings, future experiments are required to pinpoint the optimal experimental conditions for monosynaptic connectivity inference in awake rodents. Specifically, the relationship between mean firing rate and thalamic synchrony must be determined in this context. It was known that the baseline firing rate for both thalamic and cortical neurons are higher under wakefulness (Crochet and Petersen 2006; Urbain et al. 2015), however it is unclear if thalamic synchrony also increases monotonically with mean firing rate. Additionally, the effect of whisking is likely to confound the relationship between stimulus strength and measured synchrony explored in this study, hence confounding the monosynaptic connectivity inference. However, given the techniques and experimental parameters explored in this study, we believe that this has laid the foundation for capturing connectivity during wakefulness. Ideally, a non-synchronizing stimulus (sensory or optical) should be used to elevate the mean firing rate across the aligned thalamocortical brain regions while whisker videography should be in place to record whisker movement. Given that the effects of whisking on thalamic synchrony are unclear and could increase trial-totrial variability, epochs of whisking should likely be excluded from the analysis for monosynaptic connectivity inference.

Second, the thalamocortical circuit is built on anatomy that is well-studied, and highly convergent, with approximately 50-100 thalamic relay neurons making synapses onto a cortical layer 4 neuron with a clear topography (Bruno and Sakmann 2006). This convergent nature of the thalamocortical projections provides significant support in assessing possible connectivity, in contrast to potential connectivity across cortical laminae or other less topographically organized projections. Third, the VPm relay neurons are reported to be uniformly excitatory, thus the pre-synaptic spiking enhances the likelihood of action potentials in the post-synaptic cortical target. Although long-range projections typically tend to be excitatory rather than inhibitory, analogous approaches should certainly be developed for inhibitory connectivity that is becoming increasingly 
1210 acknowledged to play a complex and pivotal role in controlling network dynamics 1211 (Isaacson and Scanziani 2011).

1212

1213 With the scaling up of electrophysiological recordings comes challenges. In this work, we 1214 performed highly curated, small scale electrophysiological recordings to evaluate 1215 dynamics of VPm (potential pre-synaptic) and S1 (potential post-synaptic) neurons on a 1216 pair-by-pair basis as well as large-scale pre- and post-synaptic recordings, where any 1217 pair of neurons from the upstream and downstream structures would be a candidate for 1218 connectivity inference. What comes hand-in-hand with increasing recording yield that enables us to better answer questions about circuit function is an increasing diversity of recording quality, increasing diversity of cell type, and a wide array of possibilities for connectivity. In our hands, we found that the odds of finding putative monosynaptic connections per recording session increased by at least two- to three-fold, as illustrated in Figure 5, where 5-10 single units can be isolated from each brain region for the connectivity inference. Previous studies suggest that high signal quality can be obtained with multi-site recording due to its better detectability of extracellular feature across closely spaced contacts (Buzsáki 2004; Gray et al. 1995; Harris et al. 2000). Hence, this increase in recording yield as well as the increased probability of detecting monosynaptic connectivity with high-density probe recordings were likely due to improved single unit isolation over time, enabling reliable tracking of single units over longer recording duration (3-4 hours). Yet, in practice, handling large amounts of data with probe recordings presents challenges as most of the spike sorting algorithms to-date require manual curation to improve spike classification. This suggest that a certain amount of contamination of spikes from neighboring cells is to be expected. Overall, large-scale recording of neuronal activity will reduce the number of animals, reveal important information only available from monitoring the interaction of brain networks, but the variability inherent in these datasets drives the need for a more comprehensive approach

1237 for diverse neuronal ensembles. Traditionally, cross-correlation analysis of homogeneous, 1238 highly curated, small scale electrophysiological recordings was primarily used to attach a 1239 binary outcome for concluding monosynaptic connection in-vivo (Bruno and Simons 2002; 1240 Fujisawa et al. 2008; Miller et al. 2001; Reid and Alonso 1995; Swadlow 2003; Swadlow 1241 and Gusev 2001) and data with uncertainties were often discarded. In this work, the 1242 evaluation of the neuronal data would not result in a binary classification of connected or 1243 not, but instead the assignment of a likelihood of connectivity based on the statistical 1244 framework we present here, producing a probabilistic connectivity map. While this 1245 approach was developed through analysis of extracellular neuronal spiking data, we also 1246 envision that this would be readily adapted to optical imaging approaches that enable 1247 cellular resolution calcium or voltage imaging of population spiking activity. Recently, 1248 model-based approaches to improve connectivity estimation are being developed for the 
1249 analysis of large datasets (Chen et al. 2011; Kobayashi and Kitano 2013; Kobayashi et 1250 al. 2019; Lütcke et al. 2013; Okatan et al. 2005; Paninski et al. 2004; Stevenson et al. 1251 2008; Zaytsev et al. 2015). These methods typically involve fitting neuronal data or 1252 correlational relationships from recordings through models (i.e. generalized linear models 1253 (GLMs)) and further aim to reconstruct neuronal circuitry to estimate functional 1254 connectivity or to decode causal flow. The statistical approach we present here could 1255 eventually be combined with these more structured network modelling approaches to 1256 provide a more comprehensive framework for assessing and understanding causal 1257 interactions in the brain. 


\section{Figure captions}

Figure 1: Experimental approach used to estimate monosynaptic connectivity between somatotopically - organized areas of the rodent somatosensory pathway.

A. Simultaneous single-unit extracellular recordings were performed in the ventral posteromedial (VPm) nucleus of the thalamus and in layer IV of primary somatosensory cortex (S1) in anesthetized rodents. Recordings were targeted to topographically aligned barreloids in VPm and barrel column in S1. Weak stimulation was applied to the whisker corresponding to the recorded barreloid/barrel column to elicit non-synchronous spiking. Putative monosynaptic connection between pairs of neurons were inferred using crosscorrelation analysis. B. Experimental procedures used to establish paired recordings involve (1) Animal preparation includes surgeries. (2) S1 mapping, (3) identification of the whisker corresponding to the recorded barreloid, (4) targeting corresponding $\mathrm{S} 1$ barrel column and layer IV, (5) data collection for assessing monosynaptic connectivity by generating spikes via whisker stimulation, repeating step (3), (4) and (5) for recording additional pairs and (6) histology.

Figure 2: Targeting topographical alignment in vivo. A. S1 targeting: Location of S1 barrels was determined using intrinsic imaging (IOS) in mice. The three images on the top are images acquired in response to separate single punctate deflections of the Beta whisker, the $\mathrm{C} 2$ whisker, and the B2 whisker, respectively. A barrel template was compared to the optical image of the brain surface through the thinned skull and fitted based on the location of the centroid of barrels to provide guidance for electrode placement. B. (Left) VPm targeting: example mean LFP amplitude of evoked response (shown by downward deflection after stimulus onset) for four stimulated whiskers in mice. Note the largest and fastest response to the A3 vibrissa, and the comparatively weak responses to the adjacent B2, B3, and C3 vibrissae, lending support for VPm localization. C. Left: Histological slice showed electrode tracks marked with fluorescent dyes (see Method: Post-mortem histology) on coronal brain sections targeting VPm (bottom) and S1 layer IV (top) in a mouse. Middle: Example raw voltage traces from extracellular recordings performed simultaneously in VPm and S1 layer IV during sensory stimulation in a rat. Mean waveforms of isolated single units from each recording site were shown (shaded region indicates one standard deviation of spike amplitude), along with spike auto-correlograms of each unit in a rat (Right). D. Left: Example adapting response of a single thalamic unit from a rat, showing mean first-spike latency in response to first and last pulse of a $8 \mathrm{~Hz}$ pulsatile, ongoing stimulus ( $1^{\text {st }}$ pulse: $5.18 \mathrm{~ms}$, last pulse: $10.7 \mathrm{~ms}$ ). Right: Population adapting response for thalamic units recorded in rats and mice $\left(1^{\text {st }}\right.$ pulse (rats): $8.39 \pm 1.87 \mathrm{~ms}$, last pulse (rats): $12.3 \pm 3.04 \mathrm{~ms}, \mathrm{n}=24$ neurons, $\mathrm{N}=12$ rats, $1^{\text {st }}$ pulse (mice): $9.37 \pm 1.39 \mathrm{~ms}$, last pulse (mice): $13.1 \pm 2.78 \mathrm{~ms}, \mathrm{n}=39$ neurons, $\mathrm{N}=4$ mice). Inset: All thalamic units that we recorded showed latency shift less than $20 \mathrm{~ms}$ (latency shift (rats) $=3.65 \pm 2.62 \mathrm{~ms}, \mathrm{n}=24$ neurons, $\mathrm{N}=12$ rats, latency shift (mice) $=$ $3.71 \pm 3.11 \mathrm{~ms}, \mathrm{n}=39$ neurons, $\mathrm{N}=4$ mice), suggested that they were thalamic VPm units. E. Left: Example peri-stimulus time histogram (PSTH, $2 \mathrm{~ms}$ binsize) for a thalamic 
1305 and cortical unit in the $30-\mathrm{ms}$ window following punctate $(600 \%$ s) whisker stimulus 1306 (indicated by green dotted line at $\mathrm{t}=0$ ). We computed mean first spike latency (FSL), 1307 defined as average latency of first spike in $30 \mathrm{~ms}$ response windows after stimulus 1308 presentation for each unit (VPm: $7.5 \mathrm{~ms}, \mathrm{S1}: 11.2 \mathrm{~ms}$ ). Right: Population mean first spike 1309 latency for all simultaneously recorded thalamic and cortical units in rats and mice (VPm (rats) : $8.30 \mathrm{~ms} \pm 1.84, \mathrm{~S} 1$ (rats): $11.40 \pm 1.77 \mathrm{~ms}, \mathrm{n}=22$ neurons, $\mathrm{N}=12$ rats; VPm (mice): $9.80 \pm 1.31 \mathrm{~ms}, \mathrm{n}=9$ neuron, $\mathrm{S} 1$ (mice): $12.6 \pm 2.06 \mathrm{~ms}, \mathrm{n}=11$ neuron, $\mathrm{N}=1$ mouse).

Figure 3: Monosynaptic connectivity inference using cross-correlation analysis. topographically aligned thalamocortical regions in-vivo resulted in binary consequences. A pair of neurons can be putatively classified as 'connected' or 'not connected', as shown in the schematic. B. Raster plots showing whisker evoked spiking response under low velocity sinusoidal stimulation (mean velocity: $25 \mathrm{deg} / \mathrm{s}$ ) for a representative example from thalamus and cortex in a rat. C. All cross correlograms were computed using VPm spike train as a reference. Occurrences of cortical spikes were measured at various time lags ( $25 \mathrm{~ms}$ window before and after a thalamic spike, with $0.5 \mathrm{~ms}$ step size; see Method: Monosynaptic Connectivity). D. Stimulus-driven cross-correlograms were constructed between the original reference VPm spike trains and the trial-shuffled cortical spike trains. E. Shuffled-corrected cross-correlograms were generated by subtracting the mean of shuffled cross correlograms (averaged from 1000 iterations) from the raw cross-

Figure 4: Evaluation of monosynaptic connection inference in the context of a signal detection framework.

1331

1332

1333

1334

1335

1336

1337

1338

1339

1340

1341

1342

1343

1344

1345

1346

1347

1348

A. In the context of signal detection framework, we defined two distinct metrics and criteria to classify neuronal pairs into 'connected' and 'not-connected' distribution. Showing here are representative example pairs from each condition. (Top) Note the qualitative difference in raw cross correlograms $\left(\mathrm{C}_{\mathrm{R}}\right)$, one with broad and distributed spikes in crosscorrelogram (left) and another with sharp peaks (right) in 1-4 ms time lags (shaded gray). The first metric is the maximum peak of the raw cross-correlogram $\left(C_{R}\right)$, and Criterion 1 was fulfilled if the maximum peak of the raw cross-correlograms was within 1-4 ms lag. (Bottom) After corrected for stimulus-driven correlations, we further quantified the significance of the peaks detected in raw cross-correlogram. Hence, second metric used here is the peak height within the window of interest (1-4 ms bin), measured as maximum peak value in shuffled-corrected cross-correlogram (Csc), normalized to the number of standard deviations (\# of SDs) with respect to the shuffled distribution. Criterion 2 was fulfilled if the peak exceeded 3.5 standard deviation of the shuffled distribution. B. As expected, the majority of simultaneously recorded thalamocortical pairs exhibited peak correlation after 0 time lag ( $n=42$ pairs, rats -22 ; mice -20$)$. Histograms show the number of events in each bin of raw cross-correlograms (top) and shuffled-corrected crosscorrelograms (bottom), sorted by latency of maximum peak in the cross-correlograms. Data were normalized to maximum peak and the colors in each row show the number of events for an individual pair, normalized to maximum peak. C. Distribution of the lags of 
1350 the peak location in the raw and shuffled-corrected correlograms across all recorded pairs.

1351 Note that the peak locations for raw and shuffled-corrected correlograms were largely

1352 the same, shifted only by $1-2$ bin size $(0.5 \mathrm{~ms}$ bin). $\mathbf{D}$. Within this framework, four possible

1353 outcomes are possible. Hit: Connected and inferred to be connected. Miss: Connected

1354 but inferred to be not-connected. False Alarm: Not connected but inferred to be connected.

1355 Correct Rejection: Not-connected and inferred to be not-connected. E. In this context, we

1356 found that 11/42 pairs have putative monosynaptic connection (denoted as solid circles),

$135731 / 22$ pairs were not connected. ( $n=22$ neurons, $N=12$ rats; $n=20$ neurons, $N=1$

1358 mouse). Note that Criterion 1 was a binary classification - pairs having peak locations

1359 from their raw cross-correlograms within the 1-4ms lag pass Criterion 1, and those which

1360 do not, fail Criterion 1. For Criterion 2, the dashed vertical line represents the 3.5 SD for

1361 the peak height metric - pairs having peak height above this criterion line pass Criterion

1362 2. Only pairs that passed both Criterion 1 and 2 in Figure 4D (bottom) were classified as

1363 'connected' (anything to the right of the vertical dashed line and on the top row) and the

1364 rest were classified as 'not connected'. For each recorded pair, probability of inferring

1365 monosynaptic connection (i.e. probability of bootstrapped data satisfying Criterion 1 and

1366 2) was depicted with a color bar. Note that 1000 iterations were performed for each data

1367 set.

1368

1369

1370

Figure 5: Connectivity matrix for topographically aligned, simultaneous multi-site recordings.

1371

1372

1373

1374

1375

1376

1377

1378

1379

1380

1381

1382

1383

1384

1385

1386

1387

1388

1389

1390

1391

1392

1393

A. Thalamic (VPm) probe recording. Five whisker-responsive thalamic units were isolated from a 32-channel silicon probe site labeled A-D. Mean waveforms of single units were shown on the right (shaded region indicates one standard deviation of spike amplitude) $(\mathrm{n}=5$ neurons). B. Cortical (S1) probe recording. Four whisker-responsive units, putatively from layer IV barrel cortex, were isolated from a 32-channel silicon probe site labeled A-D. Mean waveforms of units were shown on the right (shaded region indicates one standard deviation of spike amplitude) ( $n=4$ neurons). Table 1 shows binary outcomes of the monosynaptic connectivity inference based on Criterion 1 and 2 of crosscorrelation analysis. Table 2 shows the connectivity matrix tabulating the probability of inferring a putative monosynaptic connection using the bootstrapping method for each thalamocortical pair in Table 1 (bootstrap iteration $=1000$ ).

Figure 6: Data-length dependence effect on monosynaptic connection inference.

A. Data-length dependence effect on connectivity inference was evaluated using a subsampling method. Data length was measured in terms of geometric spikes, which was calculated by taking the multiplication of the square roots of total VPm and S1 spikes. Random subsampling of the dataset was performed in the unit of trials with 1000 iterations for each condition. B. Representative example pair of neurons from 'not connected' distribution in a rat. C. Mean and standard deviation of peak height from crosscorrelogram were computed for each subsample of 'not-connected' example. Blue line, short data-length condition (GM: 1837 spikes). Green, long data-length condition. D. Distribution of peak height after bootstrapping for two data-lengths labeled in part I, [Blue (GM): 1837 spikes; Green (GM): 4730 spikes]. E. Probability of outcome for this example,

1394 CR: Correct Reject; FA: false alarm. F- I. Similar to B-E but for 'connected' example in a 
1395

1396

1397

1398

1399

1400

1401

1402

1403

1404

1405

1406

1407

1408

1409

1410

1411

1412

1413

1414

1415

1416

1417

1418

1419

1420

1421

1422

1423

1424

1425

1426

1427

1428

1429

1430

1431

1432

1433

1434

1435

1436

1437

1438

1439

rat [Blue (GM): 3760 spikes, hit rate: $87.7 \%$, miss rate: $12.3 \%$; Green (GM): 10201 spikes, hit and miss rate: $100 \%, 0 \%]$. J. Bootstrap estimator of bias for each data length. Scatterplot of population data for connected pairs $(n=6$ pairs, $N=3$ rats). Bold, exponential fit $\left(R^{2}=0.76\right)$. K. Variance of peak height at each data-length for pairs shown in J. Bold, $1^{\text {st }}$ order polynomial $\left(R^{2}=0.62 \%\right)$. L. Geometric mean for all connected pairs (Median: 8741 spikes, $\mathrm{n}=6$ pairs, $\mathrm{N}=3$ rats).

Figure 7: Thalamic synchrony effect on monosynaptic connection inference.

A. Raster plots from simultaneously recorded thalamic and cortical units under spontaneous (no stimulus), sinusoidal stimulus (mean velocity: $25 \mathrm{deg} / \mathrm{s}$ ) and transient inputs (1200 deg/s) in a mouse. B. Mean firing rate of thalamus (VPm, blue) and cortex (S1, red) of neuronal pairs were quantified across all three stimulus conditions. VPm: spontaneous: $1.38 \pm 0.89 \mathrm{~Hz}$, sinusoidal: $2.11 \pm 1.37 \mathrm{~Hz}$, transient: $5.05 \pm 2.85 \mathrm{~Hz}$, mean \pm SEM ( $\mathrm{n}=9$ neurons); $\mathrm{S} 1$ : spontaneous: $2.11 \pm 1.37 \mathrm{~Hz}$, sinusoidal: $2.23 \pm 1.36$, transient: $3.27 \pm 1.65 \mathrm{~Hz}$ ( $n=11$ neurons, $\mathrm{N}=1$ mouse) ( ${ }^{*}$ indicating $\mathrm{p}<0.5$, Wilcoxon signed-rank test with Bonferroni correction) C. Monosynaptic connectivity inference for one representative example of 'connected' thalamocortical pair from mouse. [Checkmark symbols indicated that the pair was being classified as putatively connected; cross symbols indicated that the pair was being classified as not connected]. D. Same as C, but for not-connected example. E. Thalamic synchrony was computed across three stimulus conditions, calculated as number of synchronous events between two thalamic units that occur within central window $( \pm 7.5 \mathrm{~ms})$. ( ${ }^{*}$ indicating $p<0.05$, Wilcoxon signed-rank test, with Bonferroni correction). Spontaneous: $0.20 \pm 0.07$; Sinusoidal: $0.19 \pm 0.06$; Transient: $0.76 \pm 0.39$, mean \pm SEM ( $\mathrm{n}=9$ neurons, 15 pairs, $\mathrm{N}=1$ mouse). $\mathbf{F}$. (Top) Raw spike cross-correlograms for the two VPm units shown in the top row of $A$ in spontaneous and transient stimulus conditions. (I. Spontaneous, III. Transient). Blue box on each crosscorrelogram represents the central window $( \pm 7.5 \mathrm{~ms})$ used for thalamic synchrony computation. Scale bar represents 100 spikes in cross-correlograms. (Bottom) The relationship between thalamic synchrony and mean firing rate of respective thalamic pairs was quantified across various stimulus conditions, including spontaneous, sinusoidal, and transient stimuli with six different velocities ( $n=5$ neurons, 10 pairs, $N=1$ mouse). Orange symbols represent thalamic synchrony across three stimulus conditions ( $\mathrm{l}$. Spontaneous, II. Sinusoidal, III. Transient). Blue line showing exponential fit of the relationship.

Figure 8: Potential errors in connectivity inference due to thalamic synchrony. A: Schematic shows conditions for two different synchrony levels, low and high. As thalamic synchrony increases, both thalamic and cortical showed an increase in firing rate accompanied by highly synchronous spiking in the local VPM population. To approximate this effect, we gradually added synchronous spikes in VPm and S1. We matched the firing rate of thalamic ( $\mathrm{N}$ spikes) and cortical cells ( $\mathrm{M}$ spikes) using experimental data and introduced the same amount of jitter $(\sigma)$ associated with a specific thalamic synchrony level to both spike trains. B. The effects of increase in synchronous firing on the 
1440 monosynaptic connectivity inference were examined with a probabilistic measure. Our 1441 simulation showed that with increasing thalamic synchrony, the probability of satisfying 1442 the criterion for monosynaptic connection decreased. Two example raw cross1443 correlograms, corresponding to low and high synchrony level, showed that a 'connected' 1444 pair (green, $p=0.3$ at synchrony level of 0.25 ) could be misclassified as 'not-connected' 1445 at a higher synchrony level (light blue, $p=0.05$ at synchrony level of 1.2 ). C. Same as $A$ 1446 but for an example not connected (NC) thalamocortical pair. We gradually increased the 1447 thalamic and cortical firing by introducing synchronous spikes from a neighboring 1448 connected pair (denoted as $\mathrm{N}$ correlated spikes for thalamic and $\mathrm{M}$ correlated spikes for cortical cell). We found that the probability of error rapidly increased with thalamic synchrony (probability of satisfying Criterion 1 exceeds 0.5 as thalamic synchrony reaches 1.5. D: The probability of incorrectly inferring connectivity significantly increased with increasing synchrony. Two example raw cross-correlograms at the corresponding thalamic synchrony level showed that a 'not-connected' pair could be misclassified as 'connected' at a higher synchrony level. Note the emergence of 'monosynaptic peaks' in raw cross-correlograms with increased synchrony. 
Ahissar E, Sosnik R, and Haidarliu S. Transformation from temporal to rate coding in a somatosensory thalamocortical pathway. Nature 406: 302-306, 2000.

Ahrens MB, Orger MB, Robson DN, Li JM, and Keller PJ. Whole-brain functional imaging at cellular resolution using light-sheet microscopy. Nature Methods 10: 413-420, 2013. Alonso J-M, and Martinez LM. Functional connectivity between simple cells and complex cells in cat striate cortex. Nat Neurosci 1: 395-403, 1998. Cat Primary Visual Cortex. The Journal of Neuroscience 21: 4002, 2001.

Barthó P, Hirase H, Monconduit L, Zugaro M, Harris KD, and Buzsáki G. Characterization of Neocortical Principal Cells and Interneurons by Network Interactions and Extracellular Features. Journal of neurophysiology 92: 600-608, 2004. Journal of Neurophysiology 111: 1132-1149, 2014.

Borden PY, Ortiz AD, Waiblinger C, Sederberg AJ, Morrissette AE, Forest CR, Jaeger D, and Stanley GB. Genetically expressed voltage sensor ArcLight for imaging large scale cortical activity in the anesthetized and awake mouse (erratum). Neurophotonics 4: 039801, 2017.

Bruno RM, and Sakmann B. Cortex is driven by weak but synchronously active thalamocortical synapses. Science 312: 1622-1627, 2006.

Bruno RM, and Simons DJ. Feedforward mechanisms of excitatory and inhibitory cortical receptive fields. The Journal of neuroscience : the official journal of the Society for Neuroscience 22: 10966-10975, 2002.

Buzsáki G. Large-scale recording of neuronal ensembles. Nat Neurosci 7: 446-451, 2004.

Buzsáki G, Stark E, Berényi A, Khodagholy D, Kipke DR, Yoon E, and Wise KD. Tools for probing local circuits: high-density silicon probes combined with optogenetics. Neuron 86: 92-105, 2015.

Castejon C, Barros-Zulaica N, and Nuñez A. Control of somatosensory cortical processing by thalamic posterior medial nucleus: a new role of thalamus in cortical Function. PloS one 11: e0148169, 2016. Chen Z, Putrino DF, Ghosh S, Barbieri R, and Brown EN. Statistical Inference for Assessing Functional Connectivity of Neuronal Ensembles With Sparse Spiking Data. IEEE Transactions on Neural Systems and Rehabilitation Engineering 19: 121-135, 2011.

Chung JE, Joo HR, Fan JL, Liu DF, Barnett AH, Chen S, Geaghan-Breiner C, Karlsson MP, Karlsson M, Lee KY, Liang H, Magland JF, Pebbles JA, Tooker AC, Greengard LF, Tolosa VM, and Frank LM. High-Density, Long-Lasting, and Multi-region Electrophysiological Recordings Using Polymer Electrode Arrays. Neuron 101: 21-31.e25, 2019.

Constantinople CM, and Bruno RM. Deep Cortical Layers Are Activated Directly by Thalamus. Science 340: 1591-1594, 2013.

Crochet S, and Petersen CCH. Correlating whisker behavior with membrane potential in barrel cortex of awake mice. Nat Neurosci 9: 608-610, 2006.

Csicsvari J, Hirase H, Czurko A, and Buzsáki G. Reliability and state dependence of pyramidal cellinterneuron synapses in the hippocampus: an ensemble approach in the behaving rat. Neuron 21: 179189, 1998. 
1503

1504

1505

1506

1507

1508

1509

1510

1511

1512

1513

1514

1515

1516

1517

1518

1519

1520

1521

1522

1523

1524

1525

1526

1527

1528

1529

1530

1531

1532

1533

1534

1535

1536

1537

1538

1539

1540

1541

1542

1543

1544

1545

1546

1547

Diamond ME, Armstrong-James M, and Ebner FF. Somatic sensory responses in the rostral sector of the posterior group (POm) and in the ventral posterior medial nucleus (VPM) of the rat thalamus. Journal of Comparative Neurology 318: 462-476, 1992.

English DF, McKenzie S, Evans T, Kim K, Yoon E, and Buzsáki G. Pyramidal Cell-Interneuron Circuit Architecture and Dynamics in Hippocampal Networks. Neuron 96: 505-520.e507, 2017.

Fiáth R, Márton AL, Mátyás F, Pinke D, Márton G, Tóth $K$, and Ulbert I. Slow insertion of silicon probes improves the quality of acute neuronal recordings. Scientific Reports 9: 111, 2019.

Fiáth R, Raducanu BC, Musa S, Andrei A, Lopez CM, van Hoof C, Ruther P, Aarts A, Horváth D, and Ulbert I. A silicon-based neural probe with densely-packed low-impedance titanium nitride microelectrodes for ultrahigh-resolution in vivo recordings. Biosensors and Bioelectronics 106: 86-92, 2018.

Franklin K, and Paxinos G. The mouse brain in stereotaxic coordinates, compact. The coronal plates and diagrams. Amsterdam: Elsevier Academic Press, 2008.

Fujisawa S, Amarasingham A, Harrison MT, and Buzsaki G. Behavior-dependent short-term assembly dynamics in the medial prefrontal cortex. Nat Neurosci 11: 823-833, 2008.

Furuta T, Kaneko T, and Deschenes M. Septal neurons in barrel cortex derive their receptive field input from the lemniscal pathway. The Journal of neuroscience : the official journal of the Society for Neuroscience 29: 4089-4095, 2009.

Gil Z, and Amitai Y. Properties of Convergent Thalamocortical and Intracortical Synaptic Potentials in Single Neurons of Neocortex. The Journal of Neuroscience 16: 6567-6578, 1996.

Ginzburg II, and Sompolinsky H. Theory of correlations in stochastic neural networks. Physical review E, Statistical physics, plasmas, fluids, and related interdisciplinary topics 50: 3171-3191, 1994.

Gollnick CA, Millard DC, Ortiz AD, Bellamkonda RV, and Stanley GB. Response reliability observed with voltage-sensitive dye imaging of cortical layer 2/3: the probability of activation hypothesis. Journal of neurophysiology 115: 2456-2469, 2016.

Gray CM, Maldonado PE, Wilson M, and McNaughton B. Tetrodes markedly improve the reliability and yield of multiple single-unit isolation from multi-unit recordings in cat striate cortex. Journal of neuroscience methods 63: 43-54, 1995.

Harris KD, Henze DA, Csicsvari J, Hirase H, and Buzsáki G. Accuracy of tetrode spike separation as determined by simultaneous intracellular and extracellular measurements. J Neurophysiol 84: 401-414, 2000.

Isaacson JS, and Scanziani M. How inhibition shapes cortical activity. Neuron 72: 231-243, 2011. Jiang X, Shen S, Cadwell CR, Berens P, Sinz F, Ecker AS, Patel S, and Tolias AS. Principles of connectivity among morphologically defined cell types in adult neocortex. Science 350: 2015.

Jouhanneau J-S, Kremkow J, Dorrn AL, and Poulet JFA. In Vivo Monosynaptic Excitatory Transmission between Layer 2 Cortical Pyramidal Neurons. Cell Rep 13: 2098-2106, 2015.

Jouhanneau J-S, Kremkow J, and Poulet JFA. Single synaptic inputs drive high-precision action potentials in parvalbumin expressing GABA-ergic cortical neurons in vivo. Nature Communications 9: 1540, 2018. Jouhanneau J-S, and Poulet JFA. Multiple Two-Photon Targeted Whole-Cell Patch-Clamp Recordings From Monosynaptically Connected Neurons in vivo. Frontiers in Synaptic Neuroscience 11: 2019. Juavinett AL, Bekheet G, and Churchland AK. Chronically-implanted Neuropixels probes enable high yield recordings in freely moving mice. bioRxiv 406074, 2018.

Jun JJ, Steinmetz NA, Siegle JH, Denman DJ, Bauza M, Barbarits B, Lee AK, Anastassiou CA, Andrei A, Aydın Ç, Barbic M, Blanche TJ, Bonin V, Couto J, Dutta B, Gratiy SL, Gutnisky DA, Häusser M, Karsh B, Ledochowitsch P, Lopez CM, Mitelut C, Musa S, Okun M, Pachitariu M, Putzeys J, Rich PD, Rossant C, 
Sun W-I, Svoboda K, Carandini M, Harris KD, Koch C, O'Keefe J, and Harris TD. Fully integrated silicon probes for high-density recording of neural activity. Nature 551: 232-236, 2017.

Kathleen Kelly GEC, Jed A. Hartings, Daniel J. Simons, M. Axonal conduction properties of antidromically identified neurons in rat barrel cortex. Somatosensory \& motor research 18: 202-210, 2001.

Kobayashi R, and Kitano K. Impact of network topology on inference of synaptic connectivity from multi-neuronal spike data simulated by a large-scale cortical network model. Journal of computational neuroscience 35: 109-124, 2013.

Kobayashi R, Kurita S, Kurth A, Kitano K, Mizuseki K, Diesmann M, Richmond BJ, and Shinomoto S. Reconstructing neuronal circuitry from parallel spike trains. Nature Communications 10: 4468, 2019. Kodandaramaiah SB, Flores FJ, Holst GL, Singer AC, Han X, Brown EN, Boyden ES, and Forest CR. Multineuron intracellular recording in vivo via interacting autopatching robots. Elife 7: e24656, 2018. Landisman CE, and Connors BW. VPM and PoM nuclei of the rat somatosensory thalamus: intrinsic neuronal properties and corticothalamic feedback. Cereb Cortex 17: 2853-2865, 2007.

Lefort S, Tomm C, Floyd Sarria JC, and Petersen CC. The excitatory neuronal network of the C2 barrel column in mouse primary somatosensory cortex. Neuron 61: 301-316, 2009.

Lepperød ME, Stöber T, Hafting T, Fyhn M, and Kording KP. Inferring causal connectivity from pairwise recordings and optogenetics. bioRxiv 463760, 2018.

Lien AD, and Scanziani M. Cortical direction selectivity emerges at convergence of thalamic synapses. Nature 558: 80-86, 2018.

London M, Roth A, Beeren L, Häusser M, and Latham PE. Sensitivity to perturbations in vivo implies high noise and suggests rate coding in cortex. Nature 466: 123-127, 2010.

Lütcke H, Gerhard F, Zenke F, Gerstner W, and Helmchen F. Inference of neuronal network spike dynamics and topology from calcium imaging data. Frontiers in Neural Circuits 7: 2013.

Mainen Z, and Sejnowski T. Reliability of spike timing in neocortical neurons. Science 268: 1503-1506, 1995.

Masri R, Bezdudnaya T, Trageser JC, and Keller A. Encoding of stimulus frequency and sensor motion in the posterior medial thalamic nucleus. J Neurophysiol 100: 681-689, 2008.

Matsumura M, Chen D-f, Sawaguchi T, Kubota K, and Fetz EE. Synaptic Interactions between Primate Precentral Cortex Neurons Revealed by Spike-Triggered Averaging of Intracellular Membrane Potentials \&lt;em\&gt;In Vivo\&lt;/em\&gt. The Journal of Neuroscience 16: 7757, 1996.

Millard DC, and Stanley GB. Anatomically based Bayesian decoding of the cortical response to intracortical microstimulation. In: 2013 6th International IEEE/EMBS Conference on Neural Engineering (NER)2013, p. 1457-1460.

Miller LM, Escabi MA, Read HL, and Schreiner CE. Functional convergence of response properties in the auditory thalamocortical system. Neuron 32: 151-160, 2001.

Nowak L, and J B. Cross correlograms for neuronal spike trains. Different types of temporal correlation in neocortex, their origin and significance. 2000, p. 53-96.

Okatan M, Wilson MA, and Brown EN. Analyzing functional connectivity using a network likelihood model of ensemble neural spiking activity. Neural computation 17: 1927-1961, 2005.

Ostojic S, Brunel N, and Hakim V. How Connectivity, Background Activity, and Synaptic Properties Shape the Cross-Correlation between Spike Trains. The Journal of Neuroscience 29: 10234-10253, 2009. Pala A, and Petersen CC. In vivo measurement of cell-type-specific synaptic connectivity and synaptic transmission in layer 2/3 mouse barrel cortex. Neuron 85: 68-75, 2015.

Paninski L, Pillow JW, and Simoncelli EP. Maximum likelihood estimation of a stochastic integrate-andfire neural encoding model. Neural computation 16: 2533-2561, 2004. 
1594

1595

1596

1597

1598

1599

1600

1601

1602

1603

1604

1605

1606

1607

1608

1609

1610

1611

1612

1613

1614

1615

1616

1617

1618

1619

1620

1621

1622

1623

1624

1625

1626

1627

1628

1629

1630

1631

1632

1633

1634

1635

1636

1637

1638

1639

Paxinos G, and Watson C. The rat brain in stereotaxic coordinates. Qingchuan Zhuge translate 32: 2007. Perkel DH, Gerstein GL, and Moore GP. Neuronal spike trains and stochastic point processes. II. Simultaneous spike trains. Biophysical journal 7: 419-440, 1967.

Pfeffer CK, Xue M, He M, Huang ZJ, and Scanziani M. Inhibition of inhibition in visual cortex: the logic of connections between molecularly distinct interneurons. Nat Neurosci 16: 1068-1076, 2013.

Raducanu BC, Yazicioglu RF, Lopez CM, Ballini M, Putzeys J, Wang S, Andrei A, Welkenhuysen M, Helleputte Nv, Musa S, Puers R, Kloosterman F, Hoof Cv, and Mitra S. Time multiplexed active neural probe with 678 parallel recording sites. In: 2016 46th European Solid-State Device Research Conference (ESSDERC)2016, p. 385-388.

Reid RC, and Alonso JM. Specificity of monosynaptic connections from thalamus to visual cortex. Nature 378: 281-284, 1995.

Rios G, Lubenov EV, Chi D, Roukes ML, and Siapas AG. Nanofabricated Neural Probes for Dense 3-D Recordings of Brain Activity. Nano Letters 16: 6857-6862, 2016.

Rossant C, Kadir SN, Goodman DFM, Schulman J, Hunter MLD, Saleem AB, Grosmark A, Belluscio M, Denfield GH, Ecker AS, Tolias AS, Solomon S, Buzsaki G, Carandini M, and Harris KD. Spike sorting for large, dense electrode arrays. Nat Neurosci 19: 634-641, 2016.

Sedigh-Sarvestani M, Vigeland L, Fernandez-Lamo I, Taylor MM, Palmer LA, and Contreras D. Intracellular, \&lt;em\&gt; In Vivo\&lt;/em\&gt;, Dynamics of Thalamocortical Synapses in Visual Cortex. The Journal of Neuroscience 37: 5250, 2017.

Sheikhattar A, Miran S, Liu J, Fritz JB, Shamma SA, Kanold PO, and Babadi B. Extracting neuronal functional network dynamics via adaptive Granger causality analysis. Proceedings of the National Academy of Sciences 115: E3869, 2018.

Sitnikova EY, and Raevskii VV. The lemniscal and paralemniscal pathways of the trigeminal system in rodents are integrated at the level of the somatosensory cortex. Neuroscience and behavioral physiology 40: 325-331, 2010.

Sosnik R, Haidarliu S, and Ahissar E. Temporal frequency of whisker movement. I. Representations in brain stem and thalamus. Journal of Neurophysiology 86: 339-353, 2001.

Stevenson IH, Rebesco JM, Miller LE, and Körding KP. Inferring functional connections between neurons. Curr Opin Neurobiol 18: 582-588, 2008.

Storchi R, Bale MR, Biella GEM, and Petersen RS. Comparison of latency and rate coding for the direction of whisker deflection in the subcortical somatosensory pathway. Journal of neurophysiology 108: 1810-1821, 2012.

Stoy WA, Kolb I, Holst GL, Liew Y, Pala A, Yang B, Boyden ES, Stanley GB, and Forest CR. Robotic navigation to subcortical neural tissue for intracellular electrophysiology in vivo. Journal of Neurophysiology 118: 1141-1150, 2017.

Swadlow H, Waxman S, and Rosene D. Latency variability and the identification of antidromically activated neurons in mammalian brain. Experimental brain research 32: 439-443, 1978.

Swadlow HA. Efferent neurons and suspected interneurons in S-1 vibrissa cortex of the awake rabbit: receptive fields and axonal properties. Journal of Neurophysiology 62: 288-308, 1989.

Swadlow HA. Fast-spike interneurons and feedforward inhibition in awake sensory neocortex. Cerebral Cortex 13: 25-32, 2003.

Swadlow HA, and Gusev AG. The impact of 'bursting' thalamic impulses at a neocortical synapse. Nat Neurosci 4: 402-408, 2001.

Temereanca S, Brown EN, and Simons DJ. Rapid changes in thalamic firing synchrony during repetitive whisker stimulation. The Journal of neuroscience : the official journal of the Society for Neuroscience 28: 11153-11164, 2008. 
1640 Urbain N, Salin PA, Libourel P-A, Comte J-C, Gentet L, and Petersen CC. Whisking-related changes in

1641 neuronal firing and membrane potential dynamics in the somatosensory thalamus of awake mice. Cell

1642 Rep 13: 647-656, 2015.

1643 Wang Q, Webber RM, and Stanley GB. Thalamic synchrony and the adaptive gating of information flow

1644 to cortex. Nat Neurosci 13: 1534-1541, 2010.

1645 Whitmire CJ, Waiblinger C, Schwarz C, and Stanley GB. Information Coding through Adaptive Gating of

1646 Synchronized Thalamic Bursting. Cell Rep 14: 795-807, 2016.

1647 Wright NC, Borden PY, Liew YJ, Bolus MF, Stoy WM, Forest CR, and Stanley GB. Rapid Cortical

1648 Adaptation and the Role of Thalamic Synchrony During Wakefulness. bioRxiv 2020.2010.2008.331660,

16492021.

1650 Yu J, and Ferster D. Functional Coupling from Simple to Complex Cells in the Visually Driven Cortical

1651 Circuit. The Journal of Neuroscience 33: 18855, 2013.

1652 Yuan J, Gong H, Li A, Li X, Chen S, Zeng S, and Luo Q. Visible rodent brain-wide networks at single-

1653 neuron resolution. Frontiers in Neuroanatomy 9: 2015.

1654 Zaytsev YV, Morrison A, and Deger M. Reconstruction of recurrent synaptic connectivity of thousands

1655 of neurons from simulated spiking activity. Journal of computational neuroscience 39: 77-103, 2015.

1656 Zhang W, and Bruno RM. High-order thalamic inputs to primary somatosensory cortex are stronger and

1657 longer lasting than cortical inputs. Elife 8: e44158, 2019. 


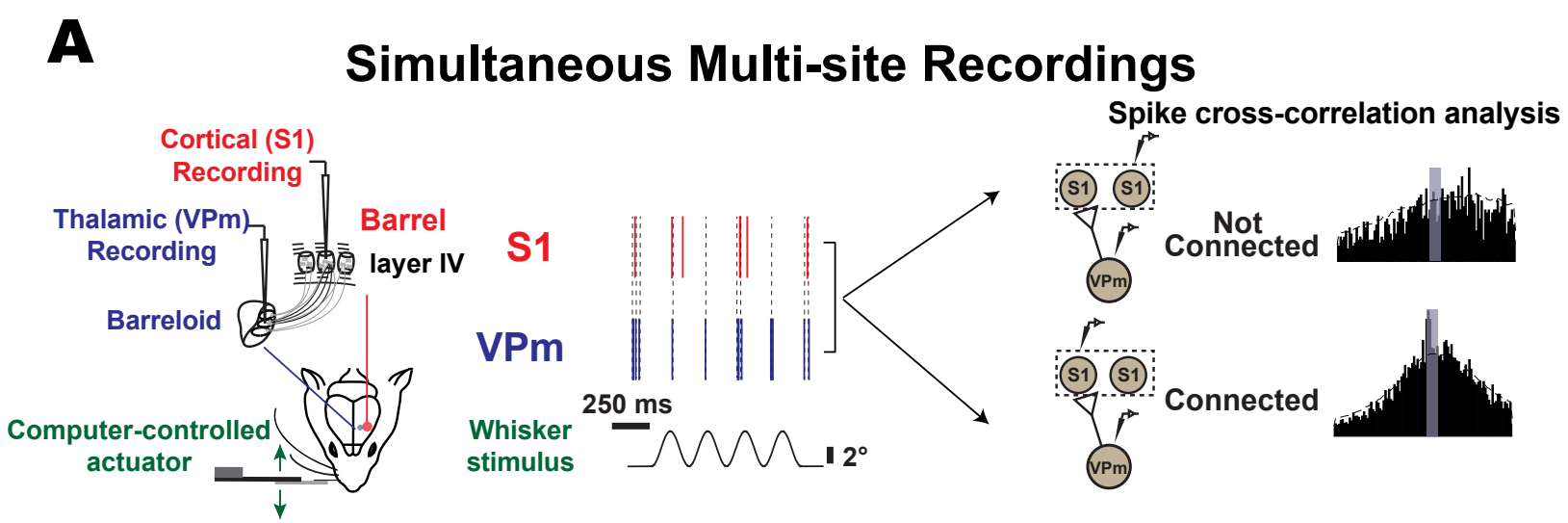

\section{B}

Experimental Workflow
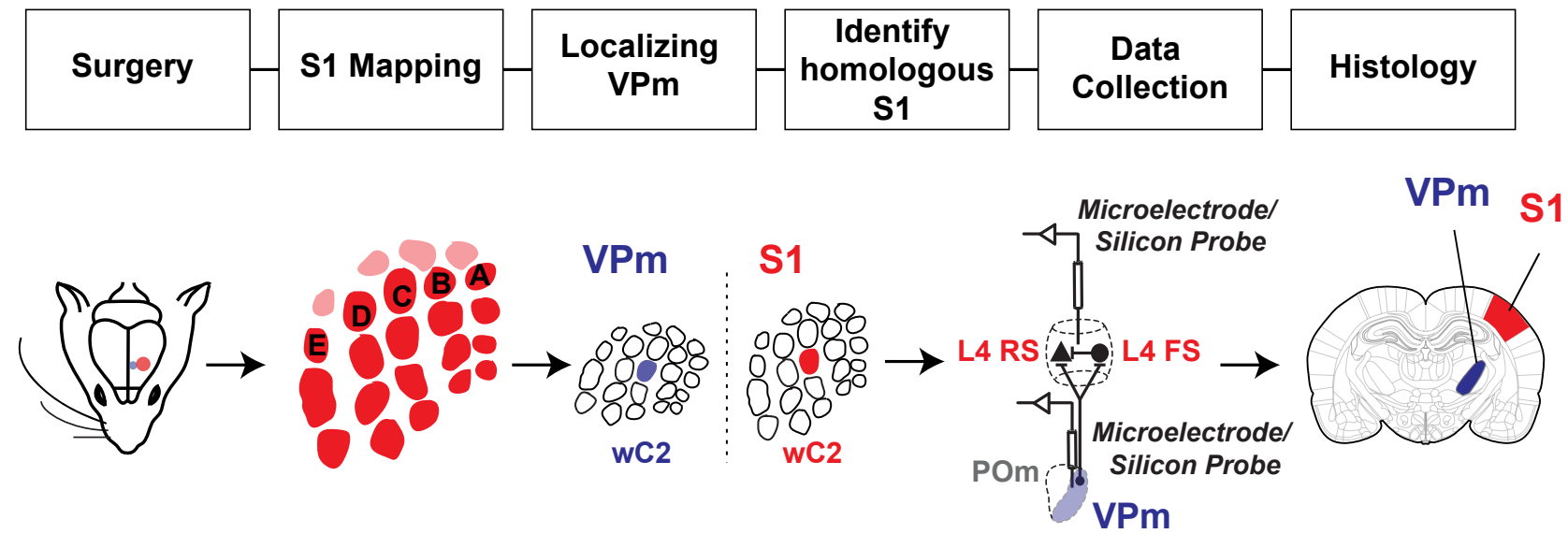

Figure 1 
A
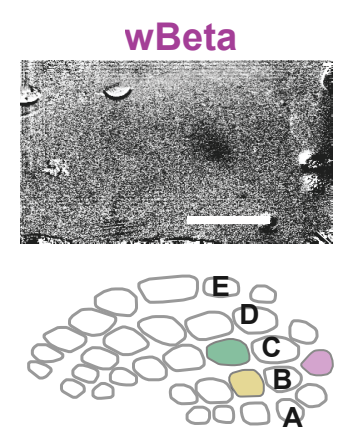

Barrel map template
S1 targeting (IOS)



B VPm targeting (LFP)
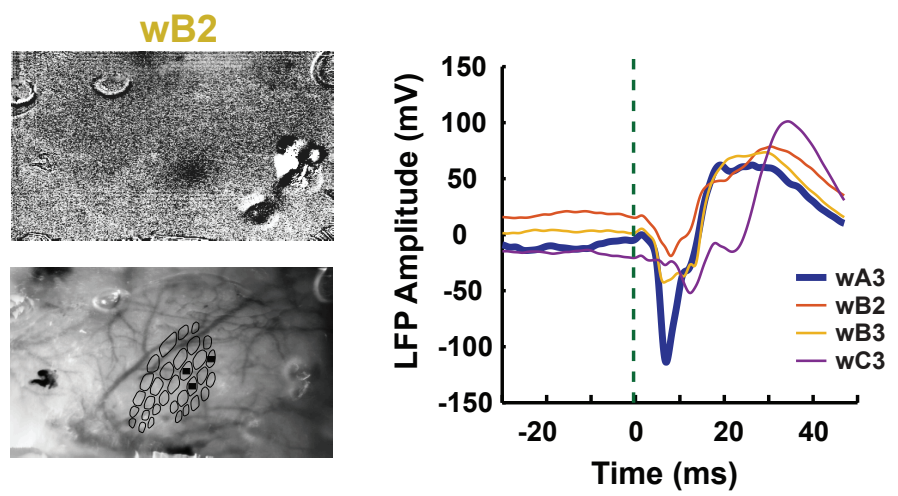

C

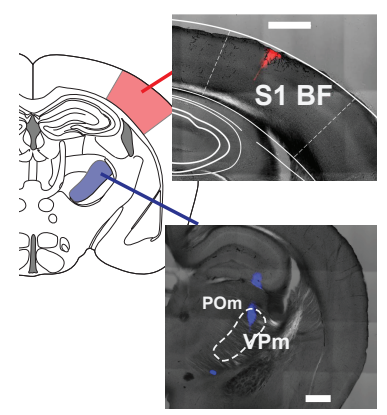

Whisker stimulus
Extracellular recording

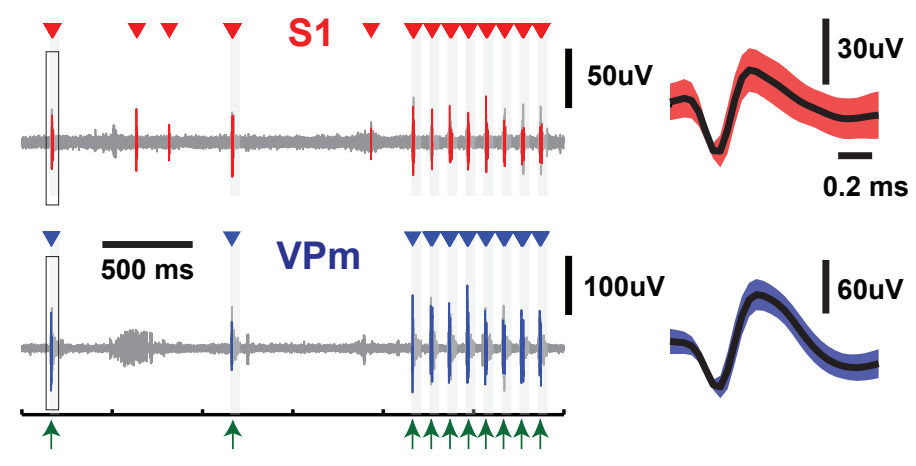

Spike waveforms

$\mathbf{E}$

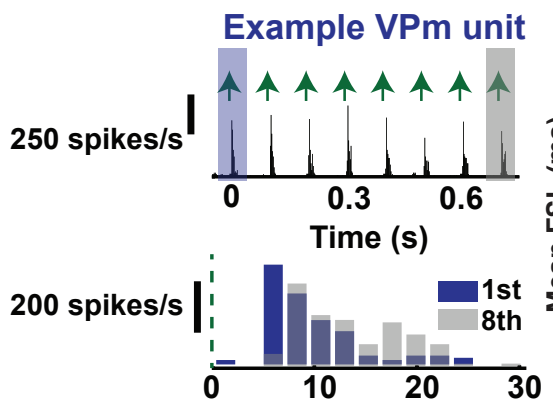

Post-stimulus time (ms)
Population VPm

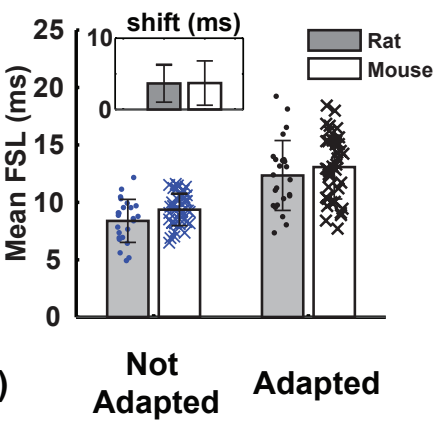

VPm-S1

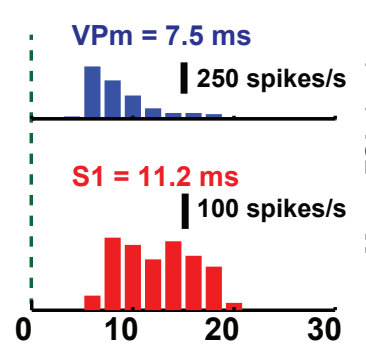

Post-stimulus time (ms)
Population VPm-S1

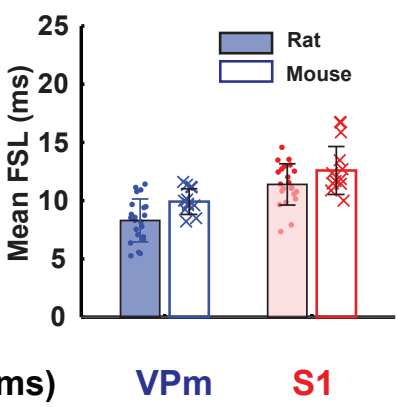

Figure 2 
$\mathbf{A}$

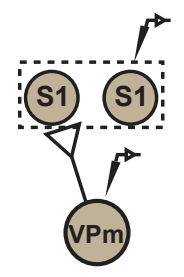

Not Connected

(NC)

C

\section{Raw cross-correlation, $C_{R}$}
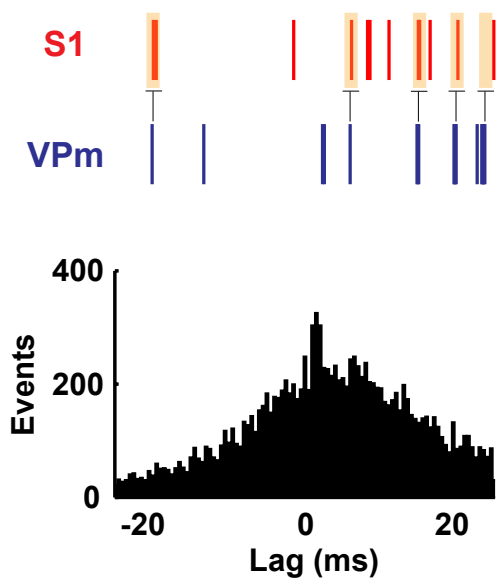

B

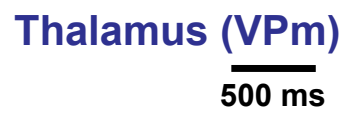

Cortex (S1)
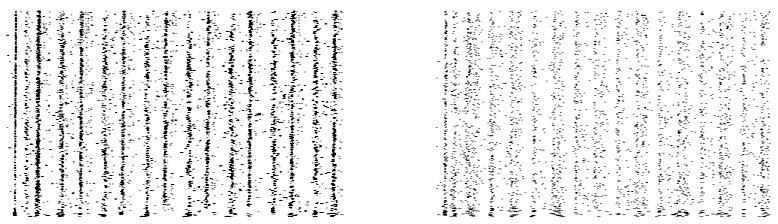

Whisker stimulus

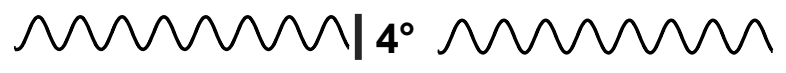

D

\section{Shuffled cross-correlation, $\boldsymbol{C}_{\boldsymbol{s}}$}
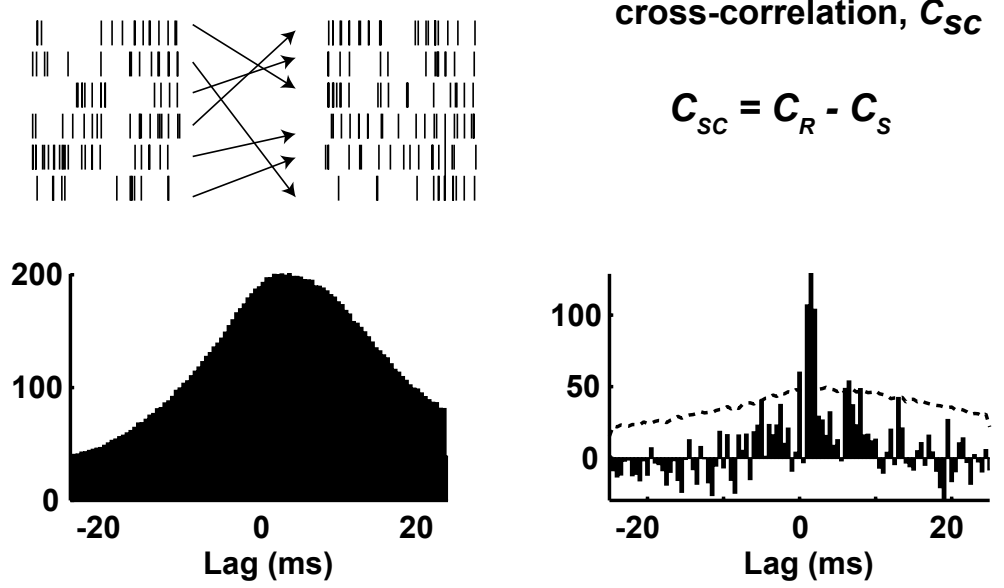

\section{Shuffled-corrected} cross-correlation, $C_{s C}$

$$
C_{s C}=C_{R}-C_{S}
$$


A Not Connected
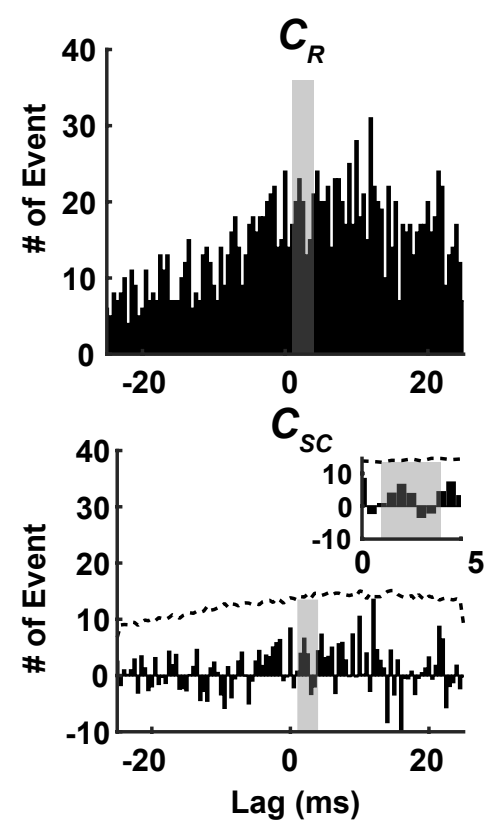

B
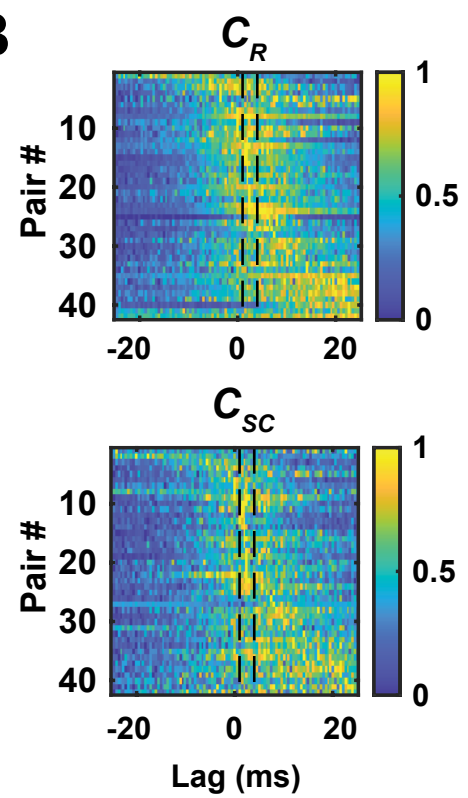

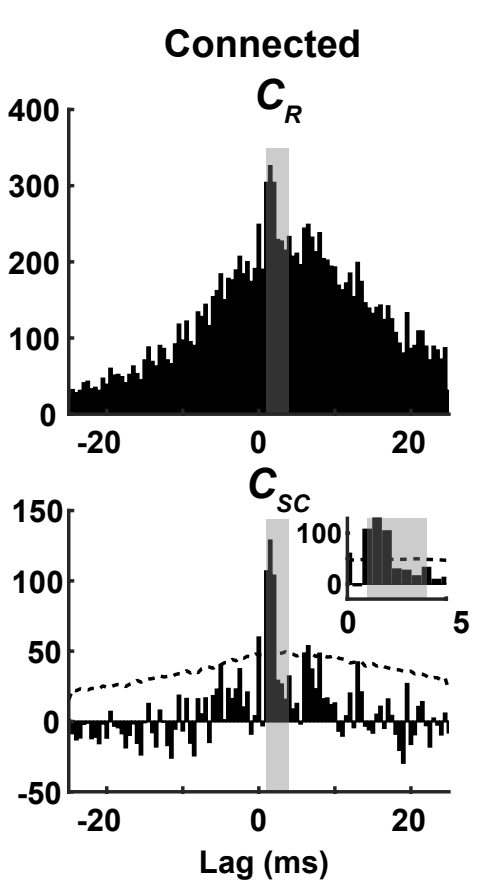

Metric: $\operatorname{Lag}_{\max \left(C_{R}\right)}$

Criterion 1:

Peak location:

$1 m s \leq \operatorname{Lag}_{\max \left(C_{R}\right)} \leq 4 m s$

Metric:

Peak height, $=\left[\frac{\max (C s c)}{s d(C s)}\right]_{1-4 m s}$

Criterion 2:

Peak height, $h \geq 3.5$

D Signal Detection

Framework

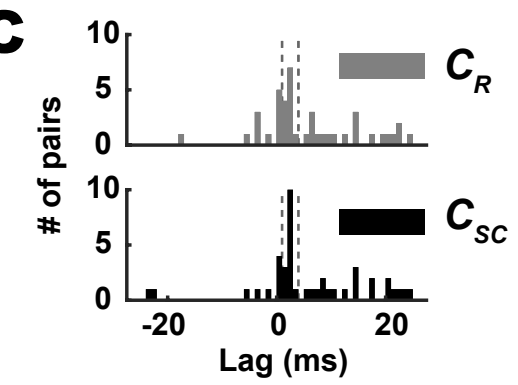

Criterion 2

E

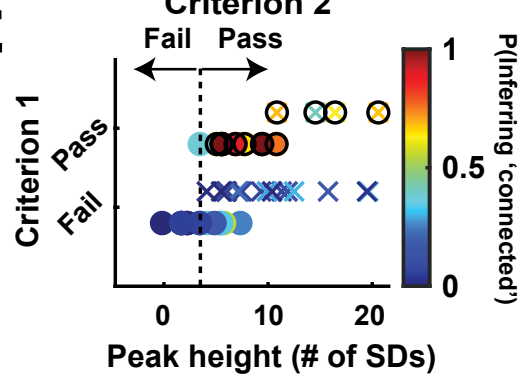

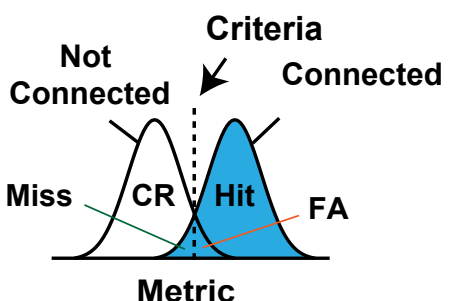

INFERENCES

Not

Connected Connected

\begin{tabular}{|c|c|c|}
\hline I Connected & HIT & MISS \\
\hline${ }_{\text {Connected }}^{\text {Not }}$ & FA & CR \\
\hline
\end{tabular}

\section{Figure 4}




\section{A}

Thalamic (VPm) Probe Recording

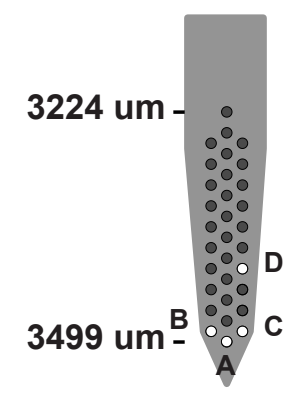

$\operatorname{VPm} 1$ (A) VPm 2 (B) VPm 3 (C)

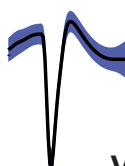

VPm 4 (C) VPm 5 (D)

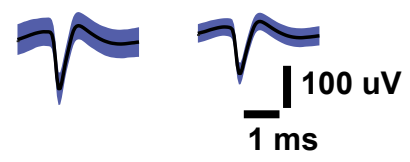

B

\section{Cortical (S1) Probe Recording}

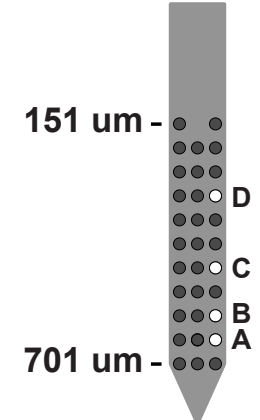

\begin{tabular}{|c|c|c|c|c|c|}
\hline \multicolumn{6}{|c|}{ Table 1 Binary Classification } \\
\hline & VPm 1 & VPm 2 & VPm 3 & VPm 4 & VPm 5 \\
\hline S1 1 & NC & C & C & NC & NC \\
\hline S1 2 & NC & NC & NC & C & NC \\
\hline S1 3 & NC & NC & NC & C & NC \\
\hline S1 4 & NC & NC & NC & NC & NC \\
\hline
\end{tabular}

Table 2 Probability of inferring 'connected'

\begin{tabular}{|c|c|c|c|c|c|}
\hline & VPm 1 & VPm 2 & VPm 3 & VPm 4 & VPm 5 \\
\hline S1 1 & 0.16 & 0.69 & 0.75 & 0.08 & 0.08 \\
\hline S1 2 & 0.18 & 0.12 & 0.37 & 0.68 & 0.03 \\
\hline S1 3 & 0.29 & 0.03 & 0.31 & 0.41 & 0.02 \\
\hline S1 4 & 0.07 & 0.08 & 0.02 & 0.23 & 0.01 \\
\hline
\end{tabular}



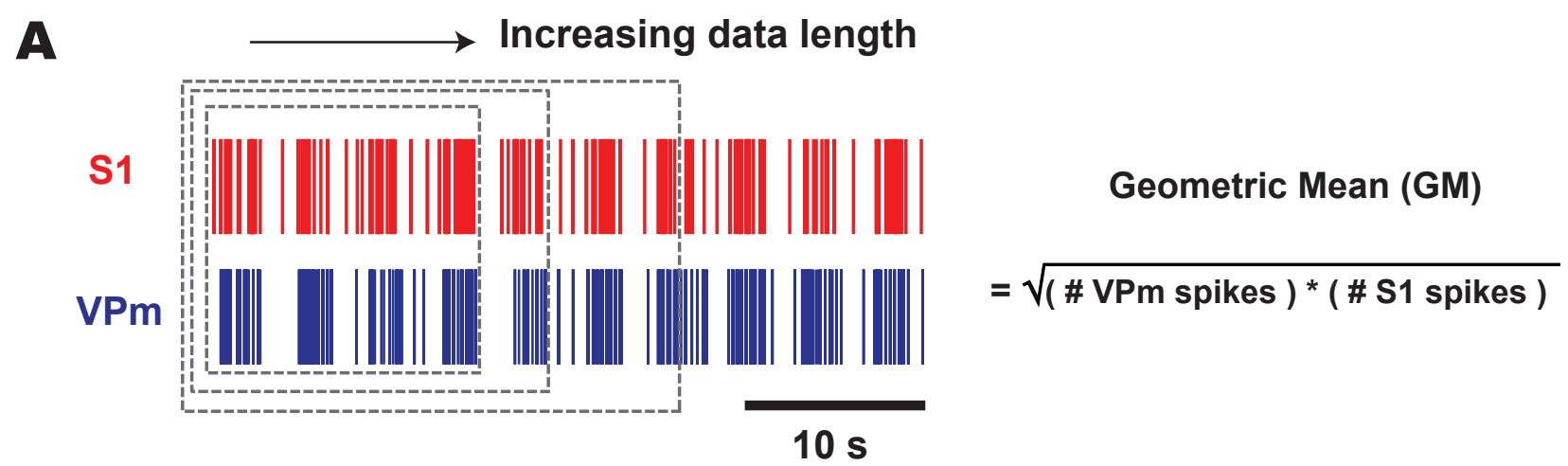

B

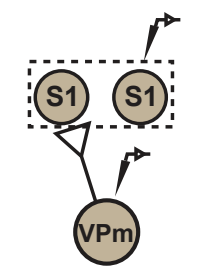

Not Connected (NC)
C

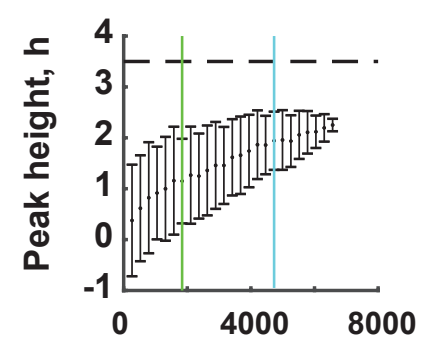

D

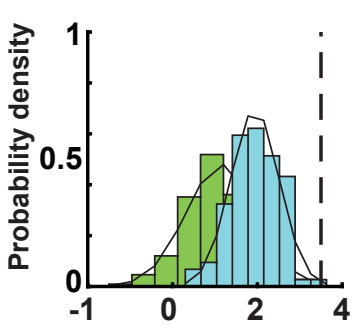

$\mathbf{F}$

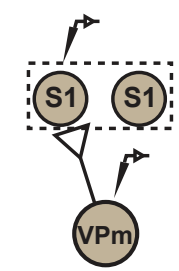

Connected (C)
G

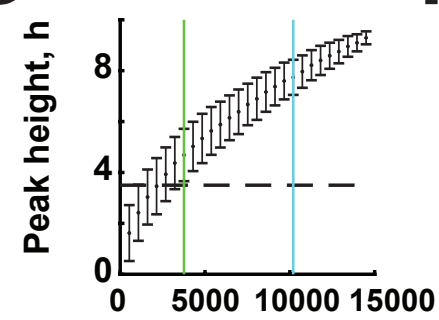

Geometric mean (spikes)

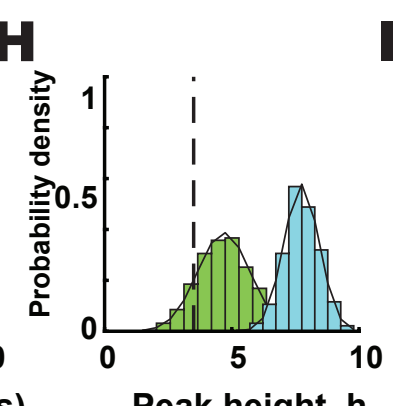

$\mathbf{E}$

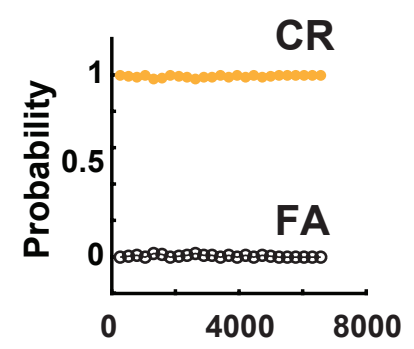

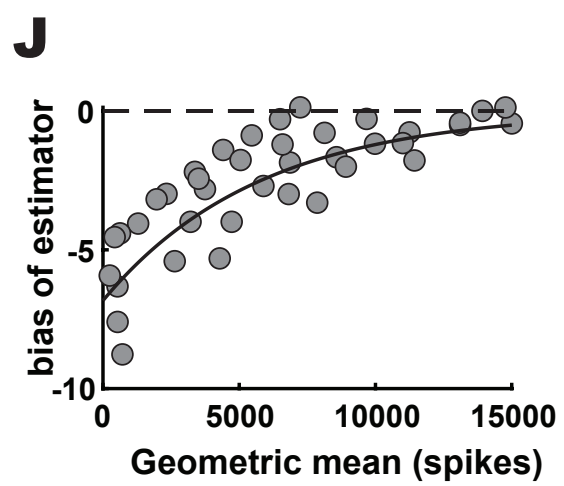
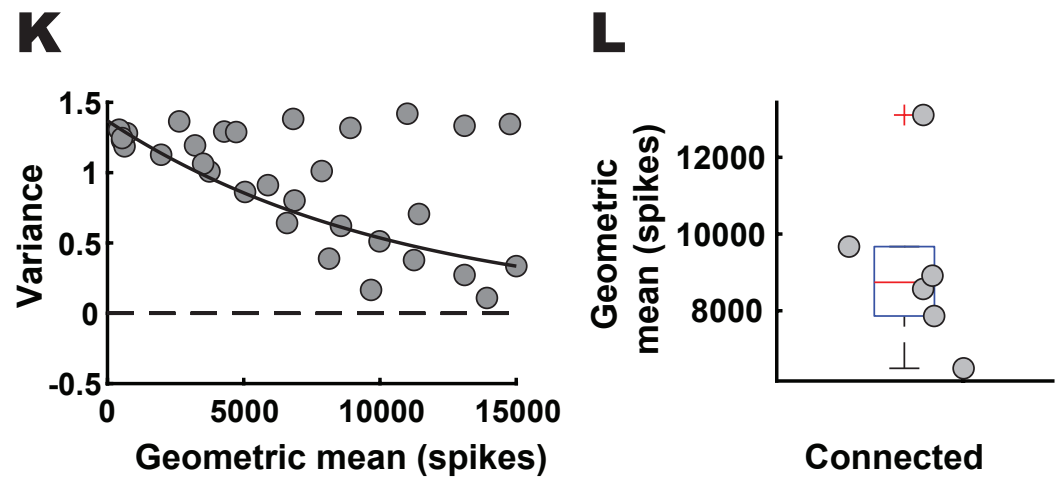

Connected

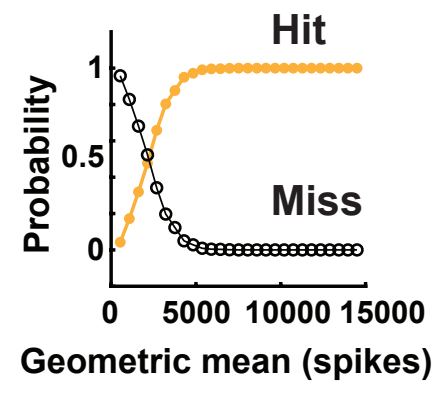

Figure 6 
$\mathbf{A}$

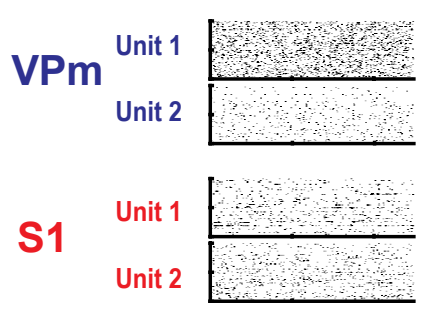

Whisker stimulus

Spontaneous
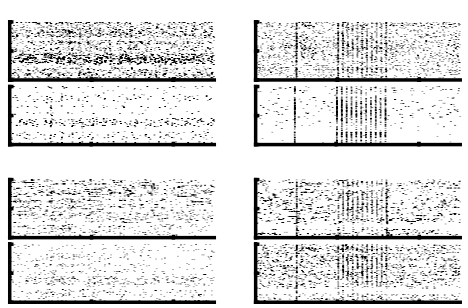

MMMMM

Sinusoidal
B

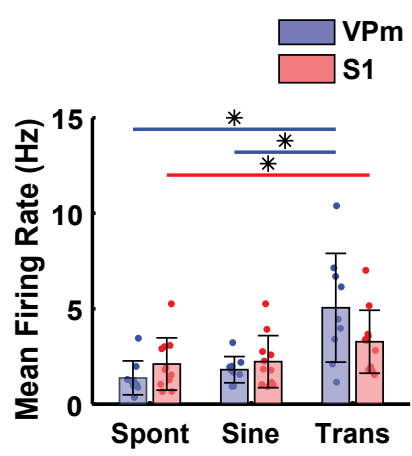

C

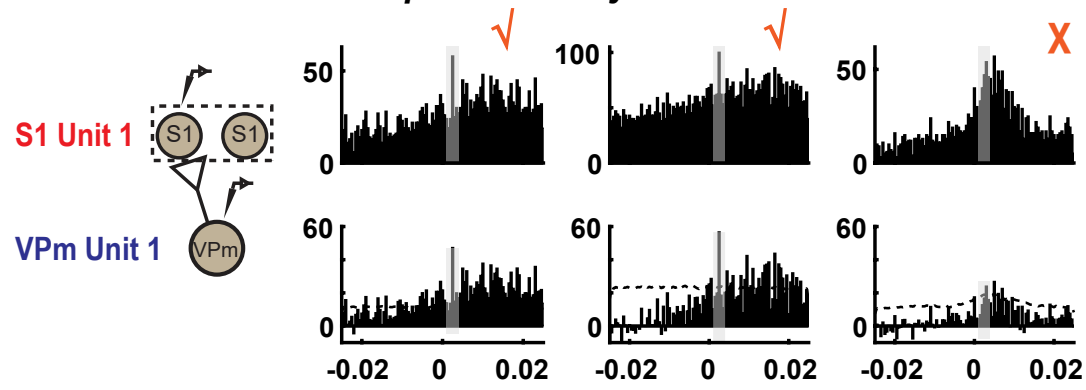

\section{Example Putatively Connected Pair}

D

\section{Example Putatively Not Connected Pair}

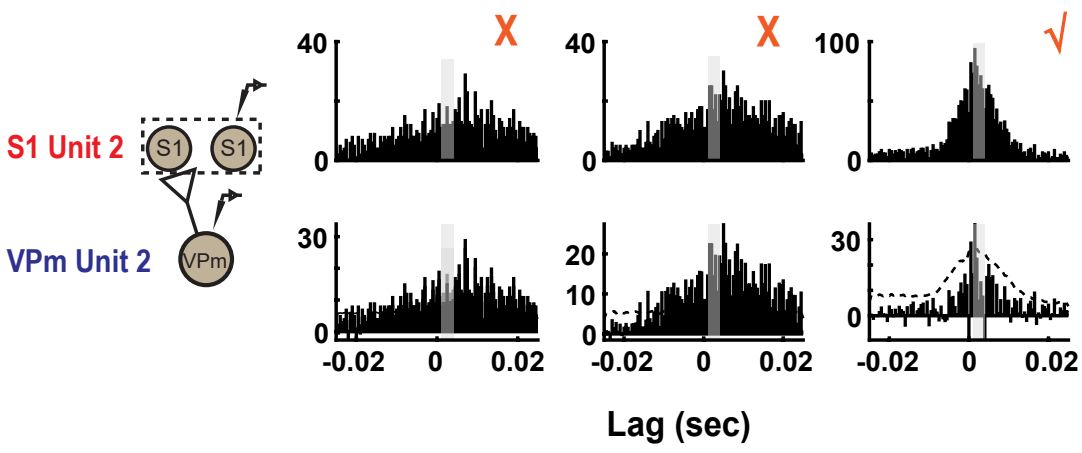

E

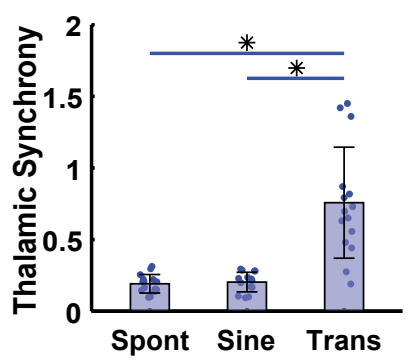

$\mathbf{F}$

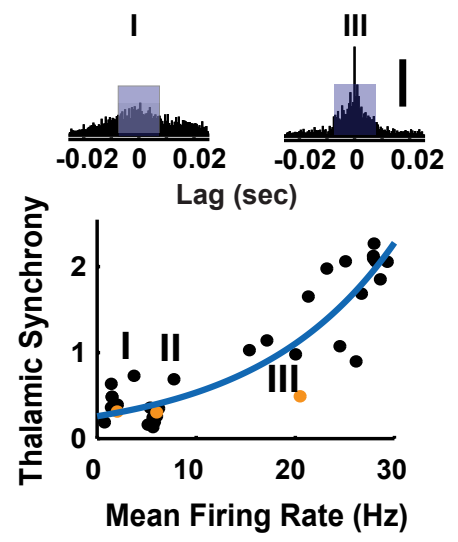


A
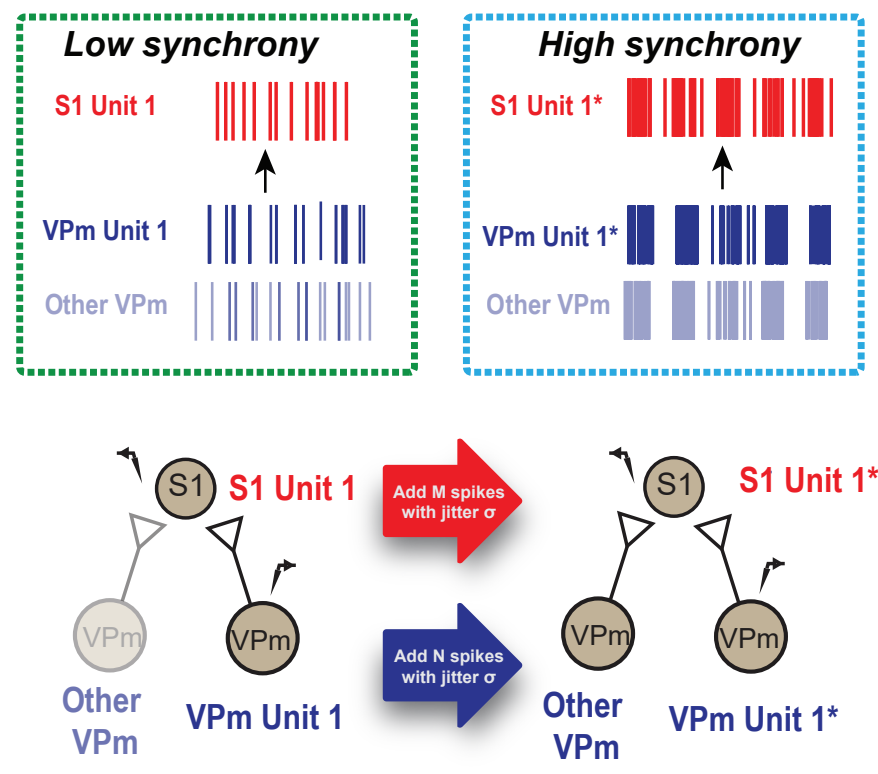

C
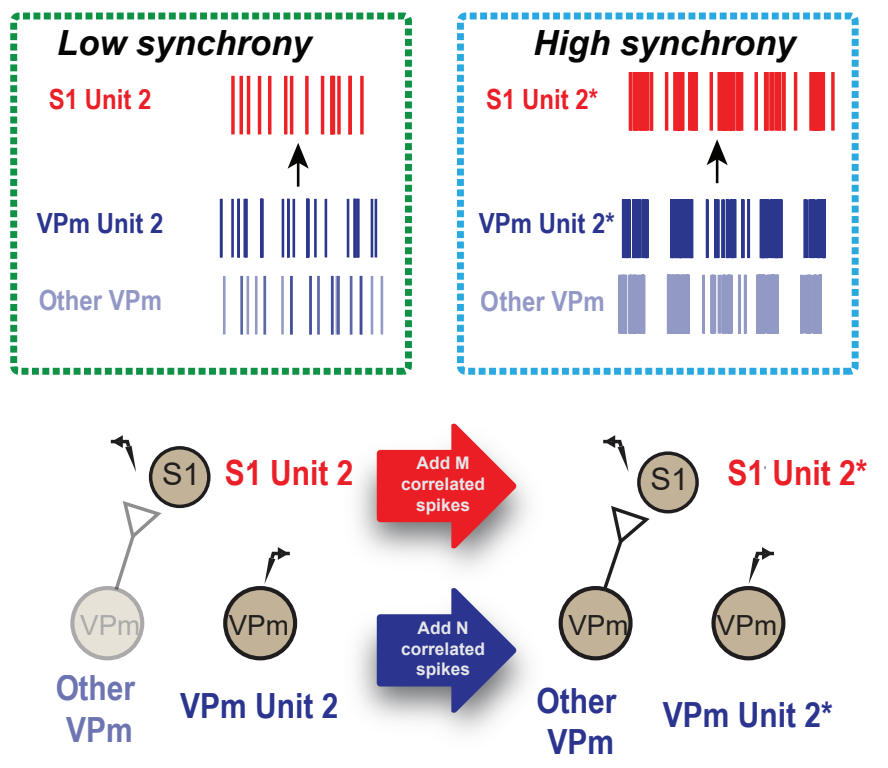

B

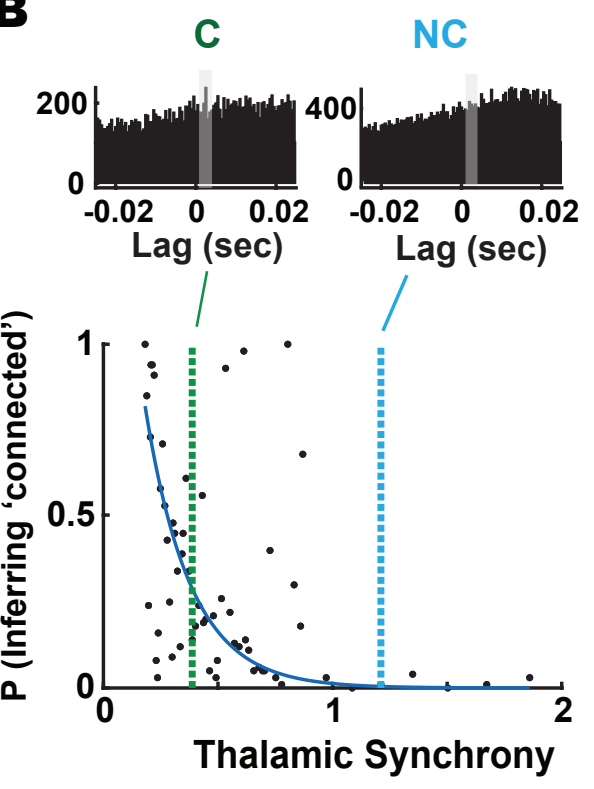

D
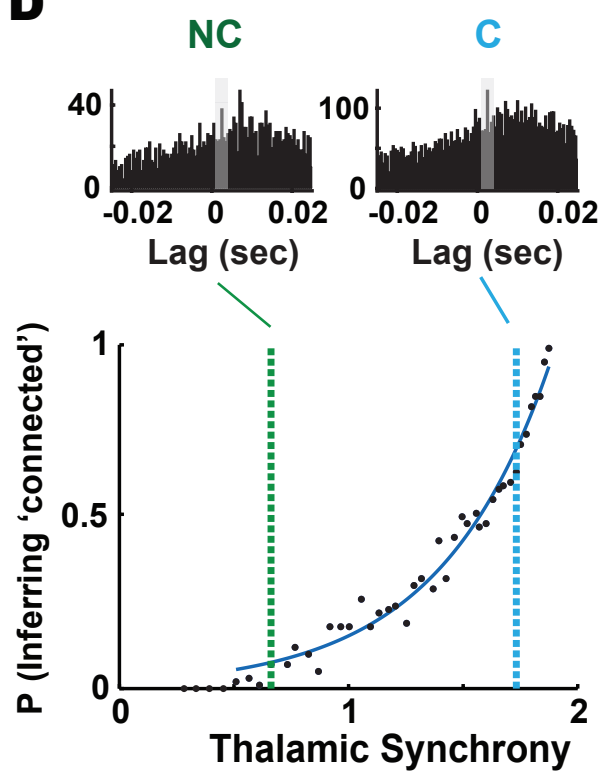

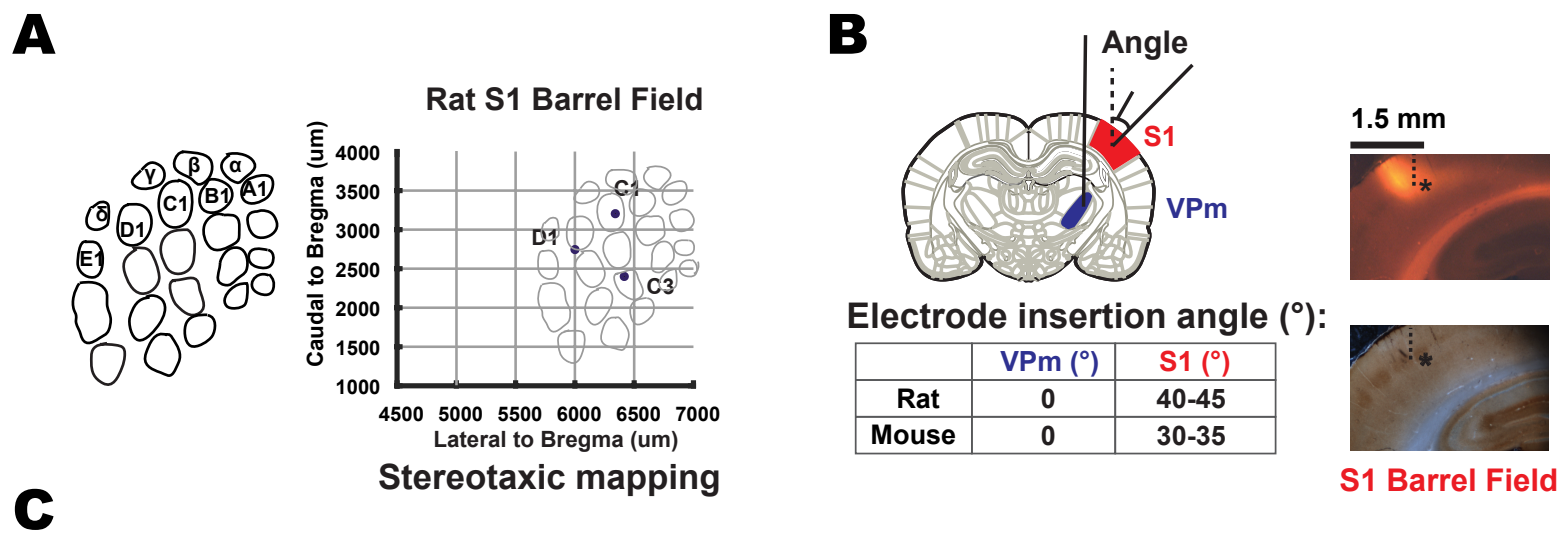

Electrode insertion angle $\left({ }^{\circ}\right)$ :

\begin{tabular}{|c|c|c|}
\hline & VPm $\left({ }^{\circ}\right)$ & $\mathrm{S} 1\left(^{\circ}\right)$ \\
\hline Rat & 0 & $40-45$ \\
\hline Mouse & 0 & $30-35$ \\
\hline
\end{tabular}
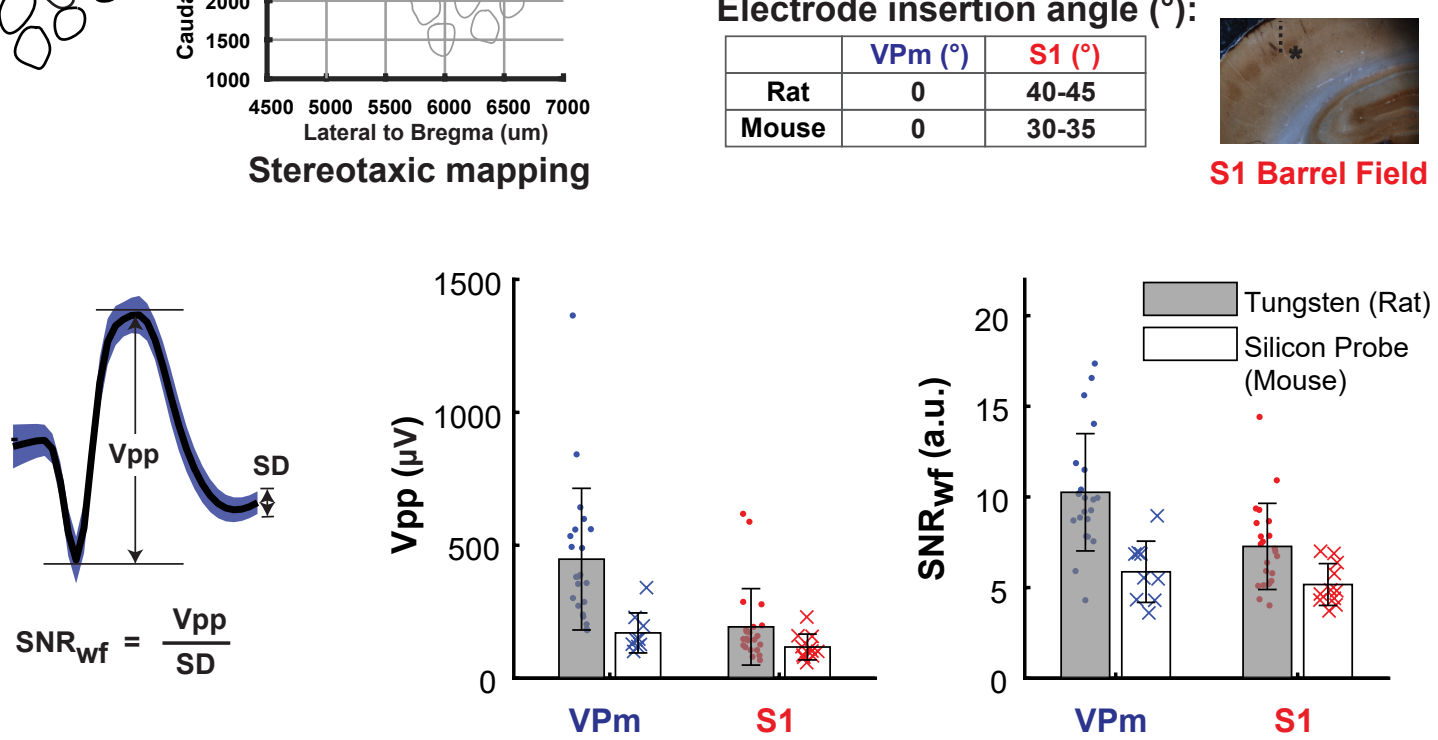

Supplementary Fig 1. VPm-S1 targeting and unit isolation quality. A. S1 targeting in rat (similar concept to IOS imaging in mouse, related to main Figure 2A): Prior to actual recording, location of $\mathrm{S} 1$ barrels was determined using functional measure (electrophysiology) and co-registered with stereotaxic coordinates relative to bregma. We mapped three distinct locations of the barrels and fitted a barrel map template using the triangulation method. This functional map was later used to guide electrode placement for targeting a desired barrel column. B. The optimal electrode insertion angle for targeting VPm and S1 in rat and mouse respectively. Histological images showed fluorescent electrode track (top) targeting S1 L4 (cytochrome oxidase staining at the bottom, see Methods) in a rat. Note: * and dotted line were plotted on both images to help registering location of fluorescent signal to barrels revealed by cytochrome oxidase staining. $C$. Schematic shows the waveform characteristics of an example single-unit. Peak-to-peak amplitude (Vpp) was defined as the voltage difference between the peak and trough of the mean waveform.

Signal-to-noise ratio $\left(S_{N R}\right.$ wf $)$ of the waveform was defined as the ratio of the peak-to-peak voltage of the mean waveform divided by the standard deviation of the waveform. Single-units recorded using Tungsten microelectrodes in rats have slightly higher peak-to-peak amplitude and signal-to-noise ratio (Vpp: VPm (rat): $448 \pm 266 \mu \mathrm{V}, \mathrm{n}=22$ neurons; VPm (mouse): $170 \pm 75.3 \mu \mathrm{V}, \mathrm{n}=9$ neurons; $\mathrm{S} 1$ (rat): $170 \pm 75.3 \mu \mathrm{V}, \mathrm{n}=22, \mathrm{~S} 1$ (mouse): $117 \pm 48.6 \mu \mathrm{V}, \mathrm{n}=11$; $\boldsymbol{S N R}_{\mathrm{wf}}$ : VPm (rat): $10.3 \pm 3.24$ a.u., $\mathrm{n}=22$ neurons; VPm (mouse): $5.87 \pm 1.69$ a.u., $\mathrm{n}=9$ neurons; $\mathrm{S} 1$ (rat): $7.27 \pm 2.38$ a.u., $\mathrm{n}=22$, S1 (mouse): $5.17 \pm 1.15$ a.u., $n=11$ ). All thalamic units have ISI violation $<2 \%$ and cortical units have ISI violation $<1 \%$. 


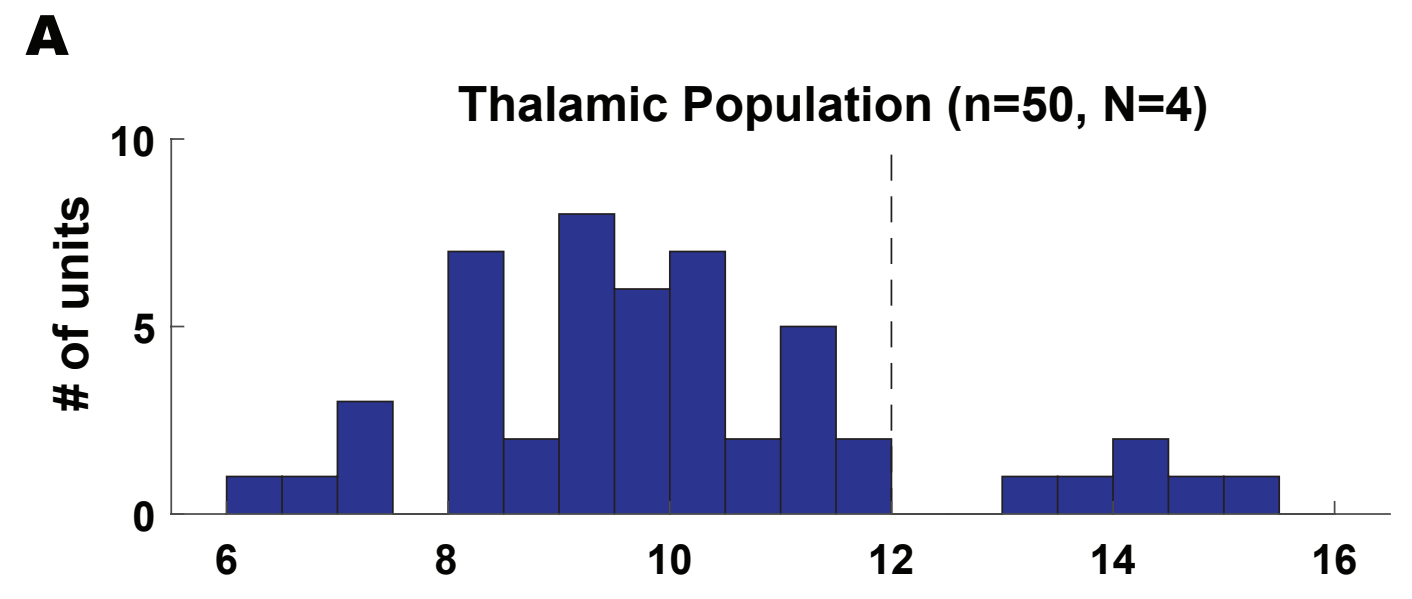

B

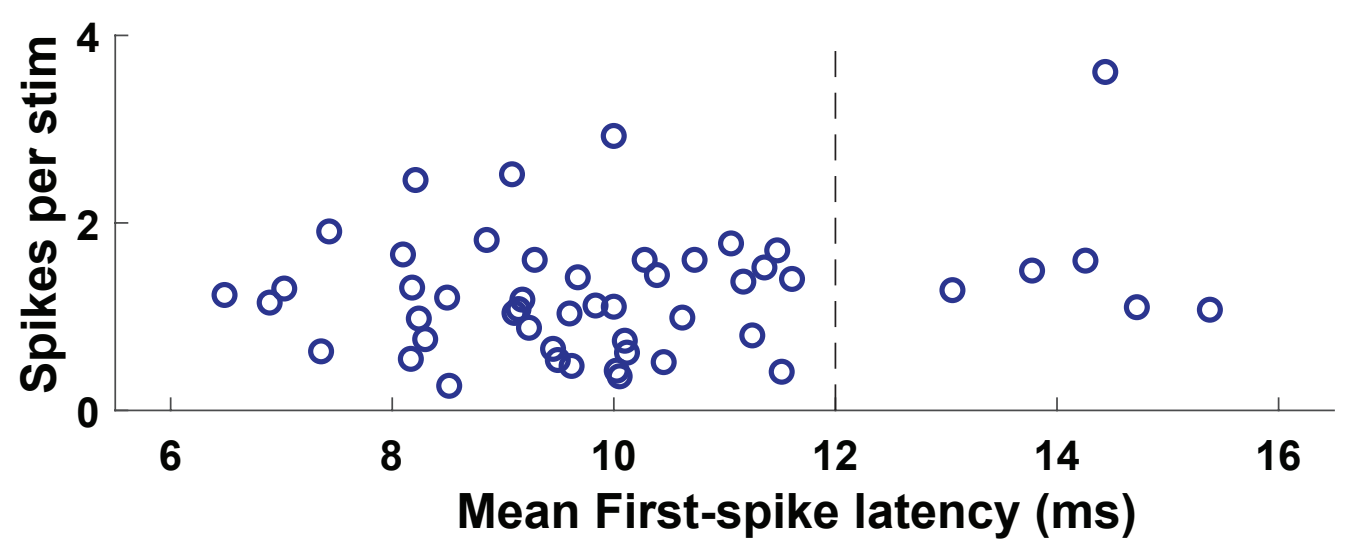

Supplementary Fig 2. Distribution of thalamic evoked responses to punctate whisker stimulation in mice. A. Distribution of mean first-spike latency of evoked responses of thalamic population in response to 1 st pulse of a repetitive $(8-10 \mathrm{~Hz})$ transient stimulation (related to Figure 2D, not-adapted response). We found that majority of these cells (44 out of 50) exhibited short first-spike latency $(9.43 \pm 1.32 \mathrm{~ms}$, mean \pm SEM). However, there were a small set of cells (6 out of 50 ) that exhibited longer latencies (13-15 ms), which could be secondary whisker responses (more likely) or POm responses (less likely). B. Corresponding averaged spikecount per stimulus as a function of mean first-spike latency for all units shown in A (1.25 \pm 0.66 spikes/stim, mean \pm SEM, $n=50$, single-unit and multi-unit data, excluding any neurons exhibiting reliability $<20 \%$ ). Dotted line depicted latency cut-off for VPm neuron classification (see Methods). 


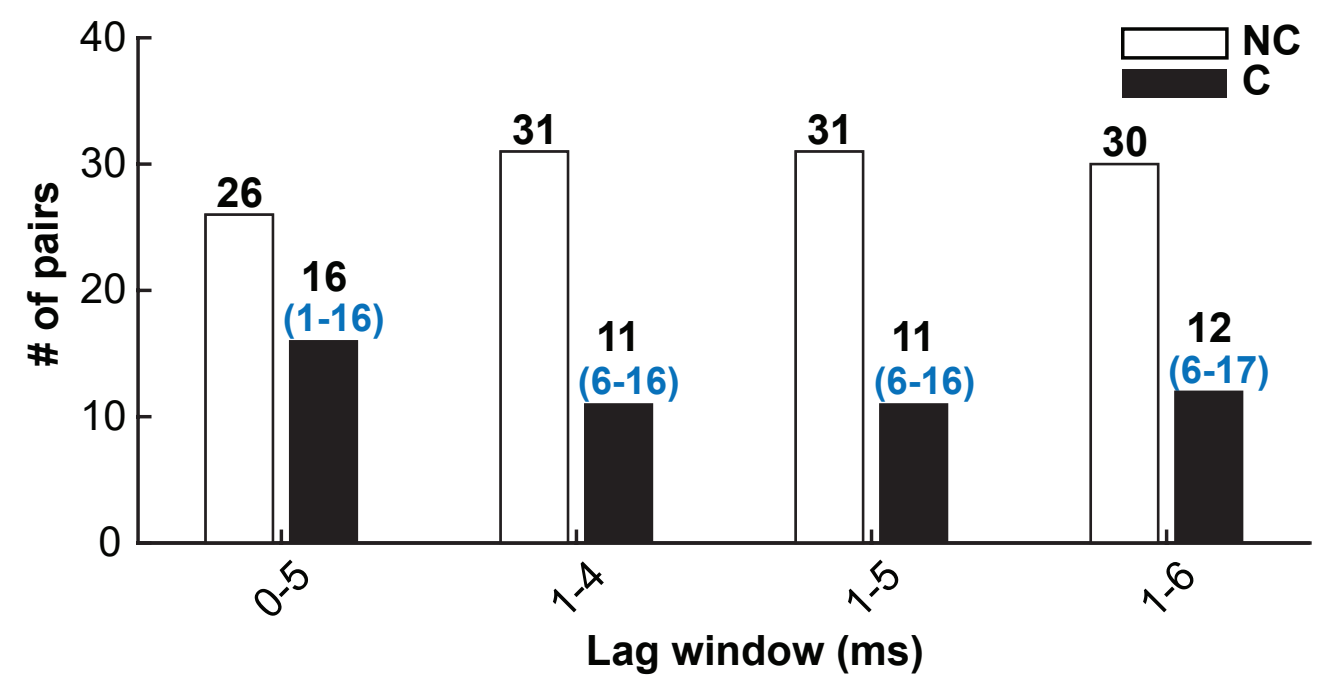

Shuffled-corrected cross-correlogram of 'connected' pairs
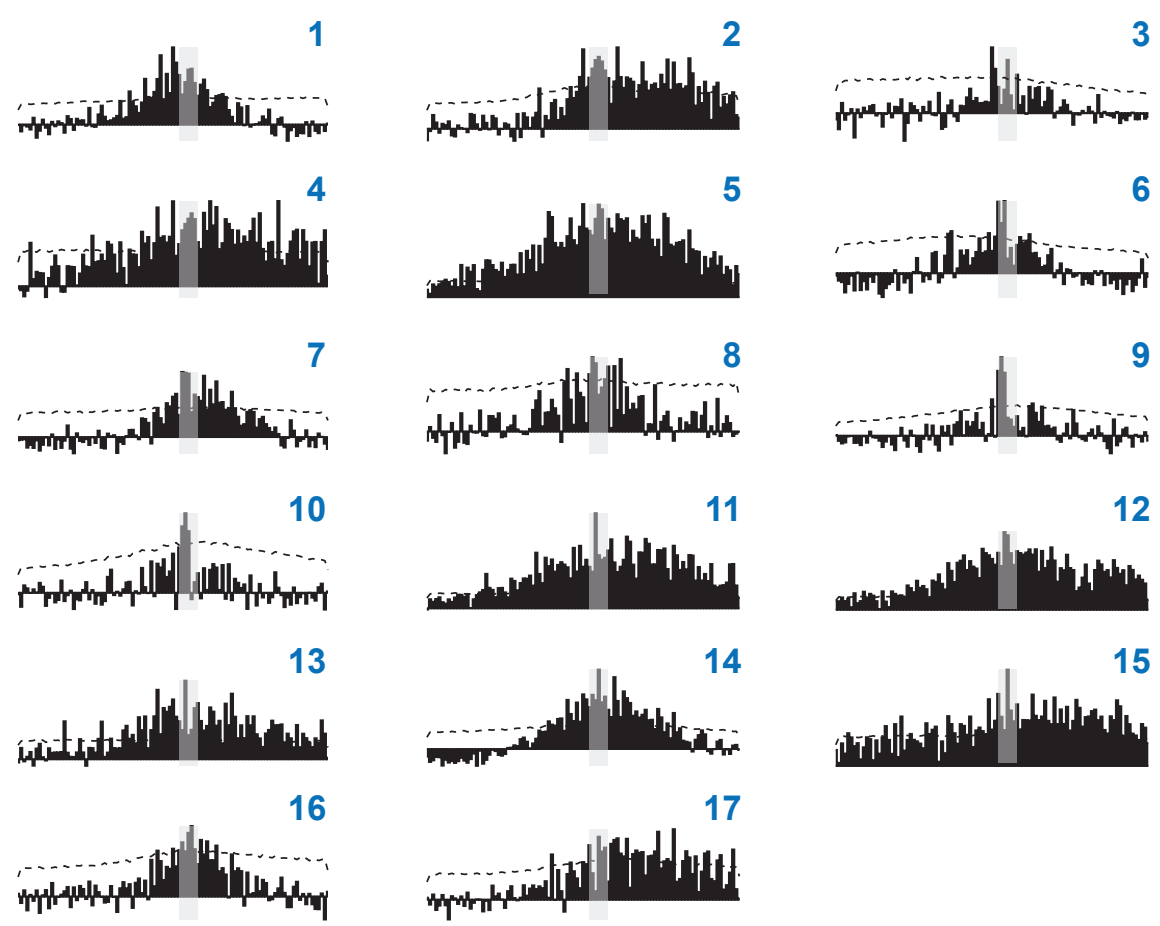

\section{7}

(n)

\section{Supplementary Fig 3. Comparison of different lag windows for connectivity analysis for} rat and mouse data. Top: Distribution of 'not-connected' (NC) and 'connected' (C) pairs based on different selections of lag window on the cross-correlograms. The parenthesized numbers (blue) correspond to a collection of indexed cross-correlograms shown below. Bottom: Shuffled-corrected cross-correlograms for 'connected' pairs, pooled across all four lag windows. Note that each cross-correlogram is indexed from 1-17, depicting the total number of 'connected' pairs by any definition of lag windows. Note that cross-correlograms (index: 6-16) are the same pairs that were classified as 'connected' in Figure 4E, also shown in Supplementary Figure 4). 
A
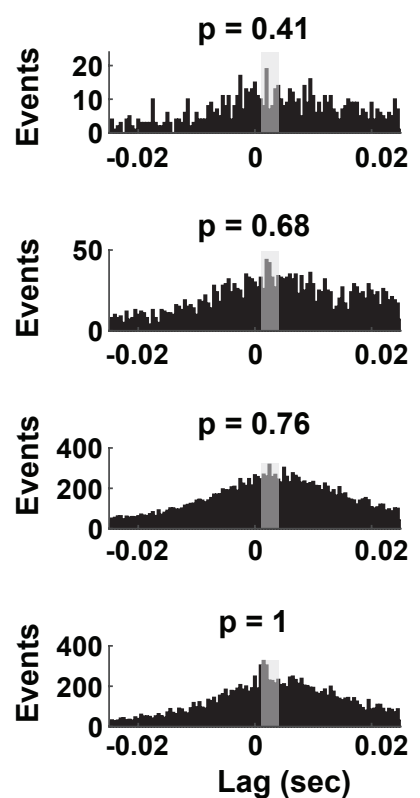

$\mathbf{B}$
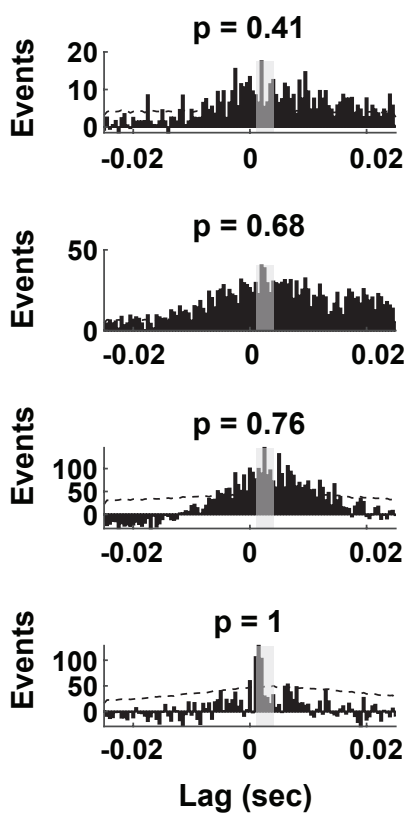
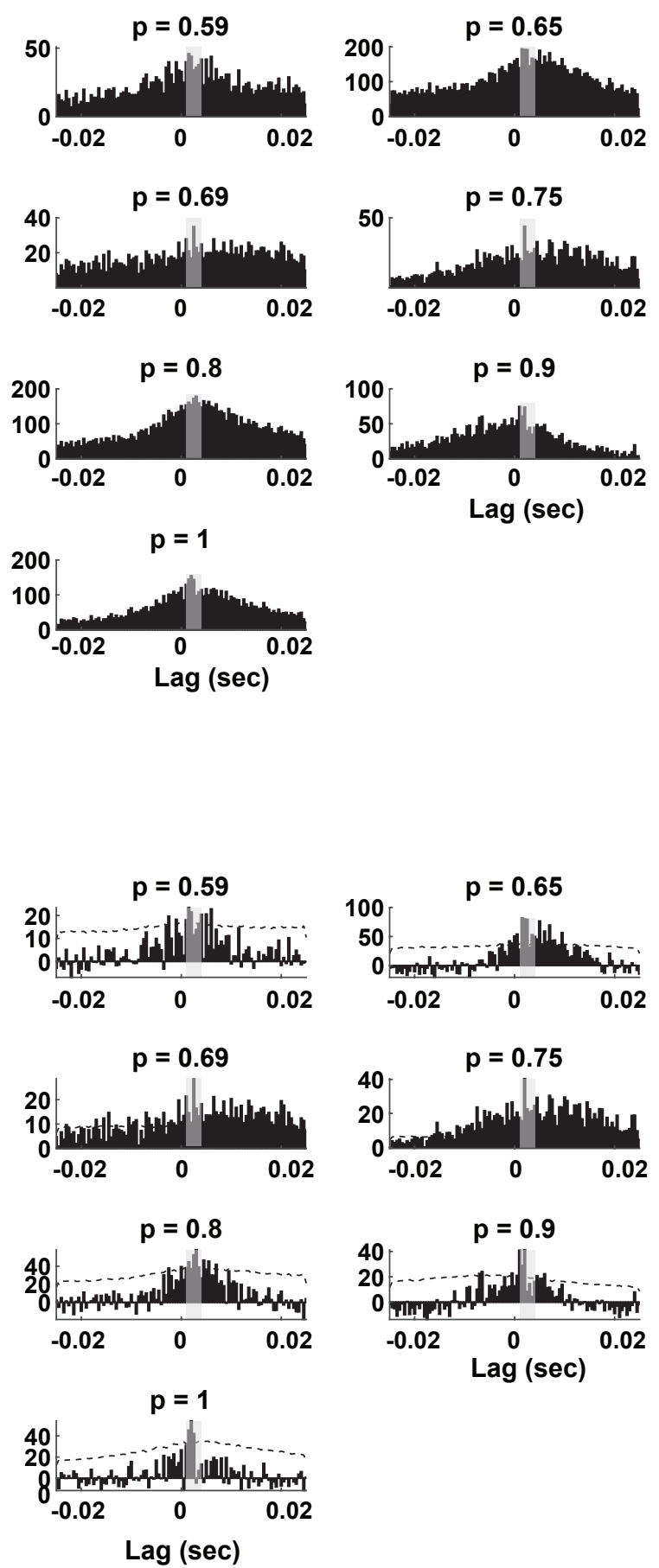

Supplementary Fig 4. Raw and shuffled-corrected cross-correlograms of 'connected' pairs in rat and mouse. A. Raw cross-correlograms of all 'connected' pairs in Figure 4E. B. Corresponding shuffled-corrected cross-correlograms in A. P-value above each plot represents the probability of a correct inference associated with each 'connected' pair (related to Figure 4 and Figure 5). 


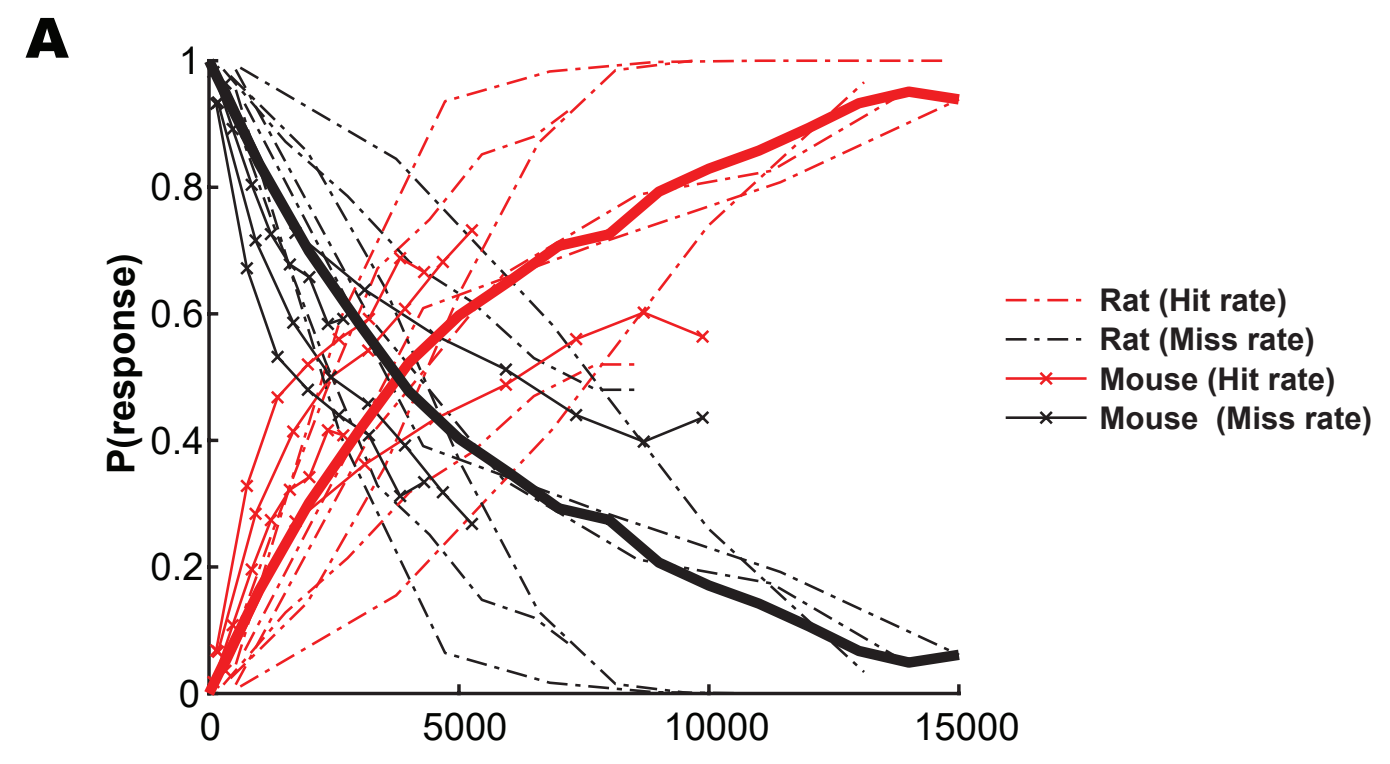

B

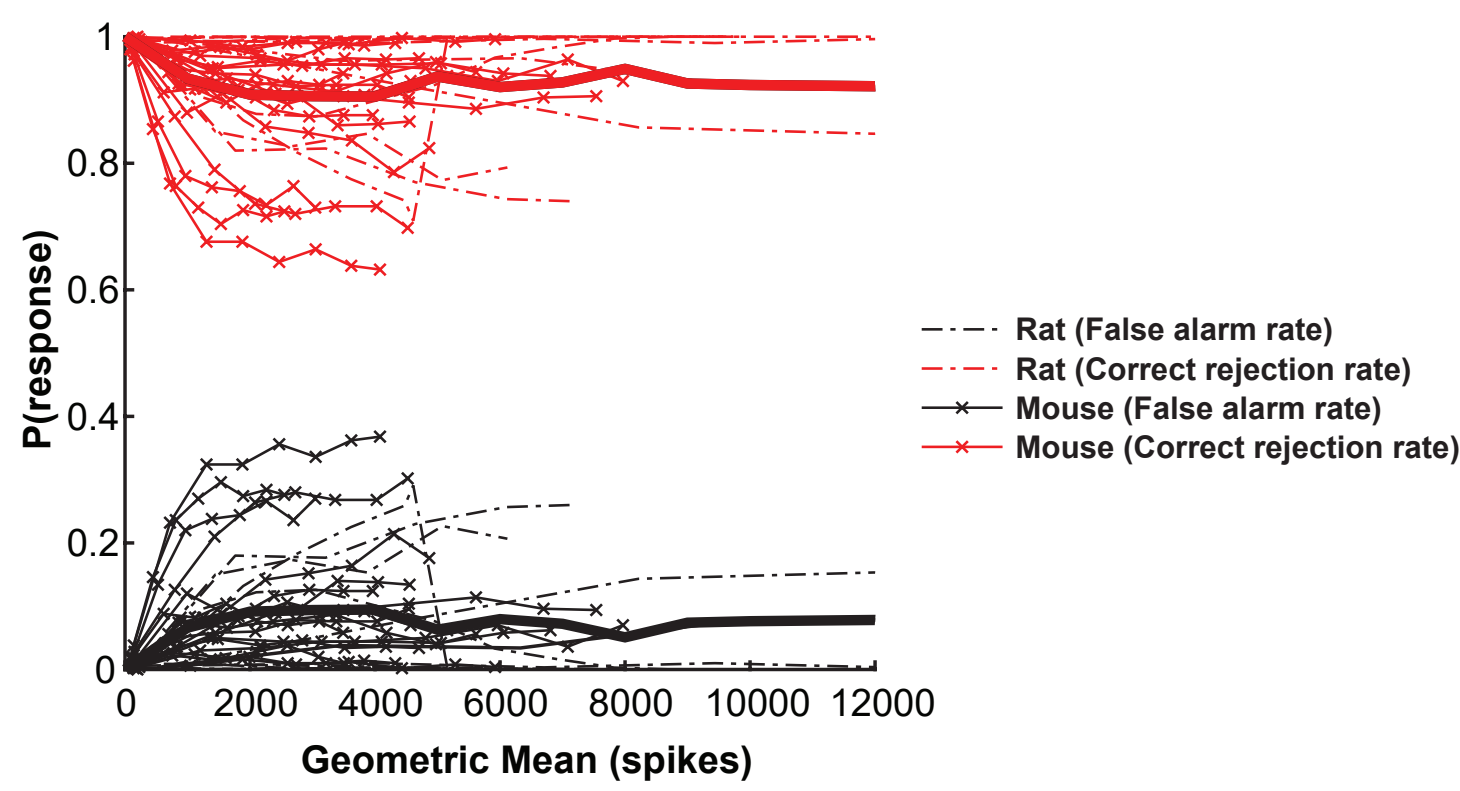

Supplementary Fig 5. Effect of data-length on monosynaptic connectivity inference for rat and mouse data. A. Hit and miss rate for all connected pairs $(n=11$ pairs). B. Correct Reject and False Alarm rate for all not-connected pairs $(n=31$ pairs). Note that the hit rate and correct reject rate are similar to $\mathrm{P}$ (correct inference) on other figures (related to Figure 6). Bold lines represent mean probabilities, averaged across pairs at each data-length (if available). 
A Site 1 Site 2 Site 3 Site $4 \quad$ B

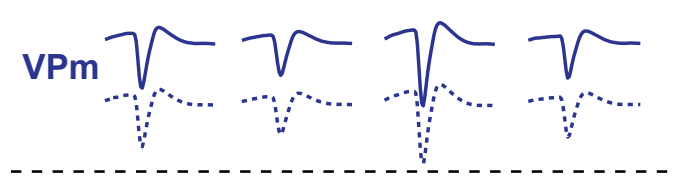

Site 3 Site 5 Site 6 Site 9
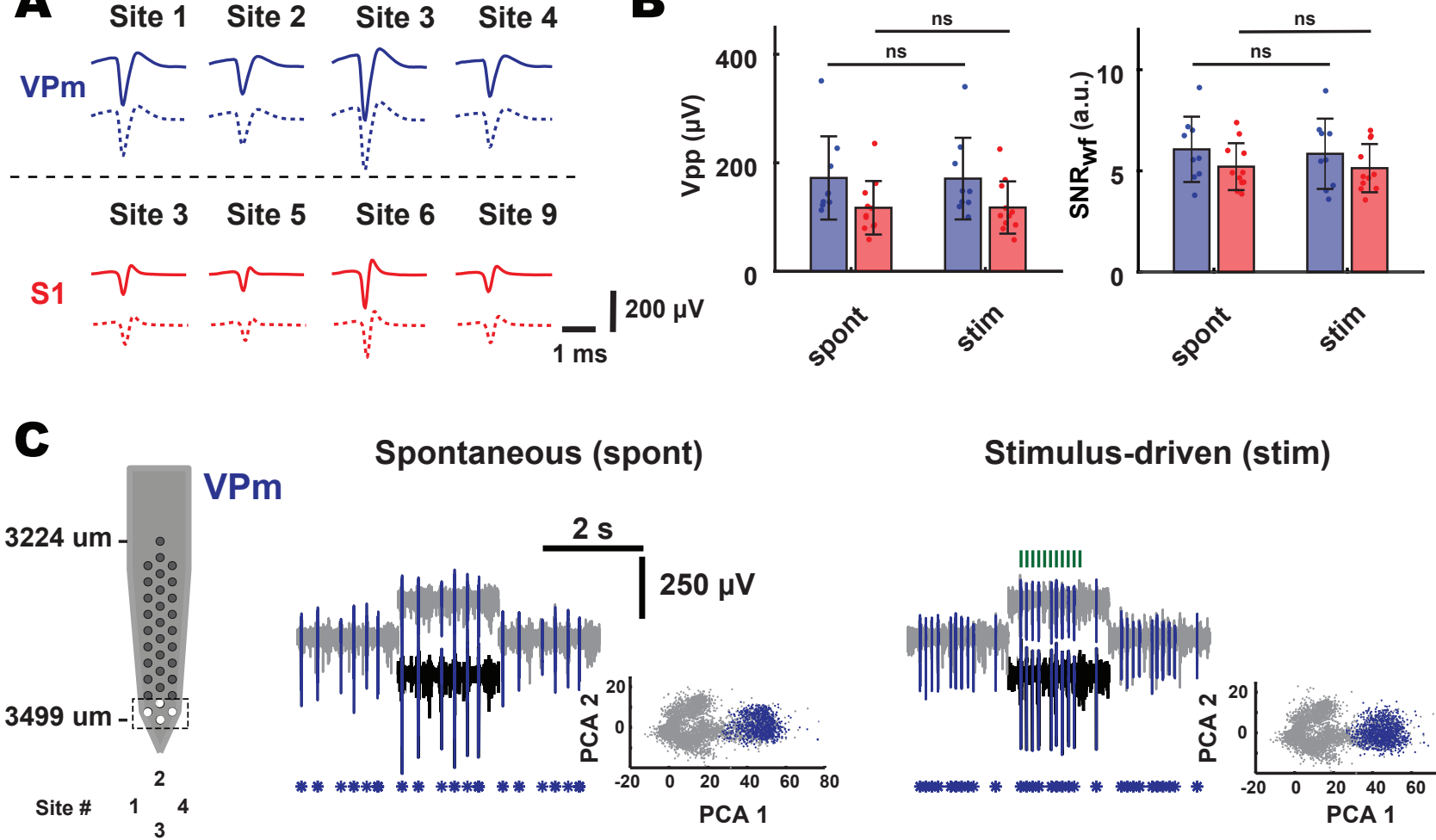

Spontaneous (spont)

Stimulus-driven (stim)

VPm
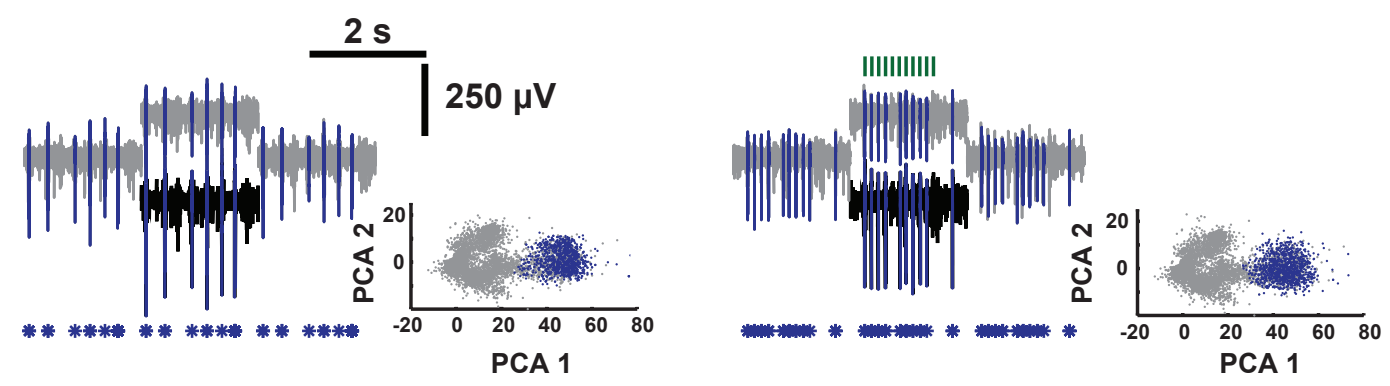

D

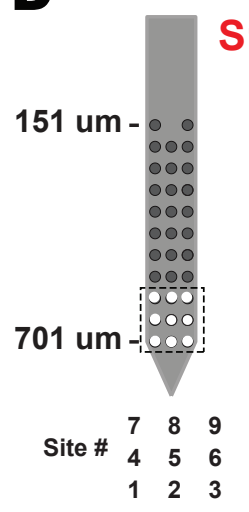

S1
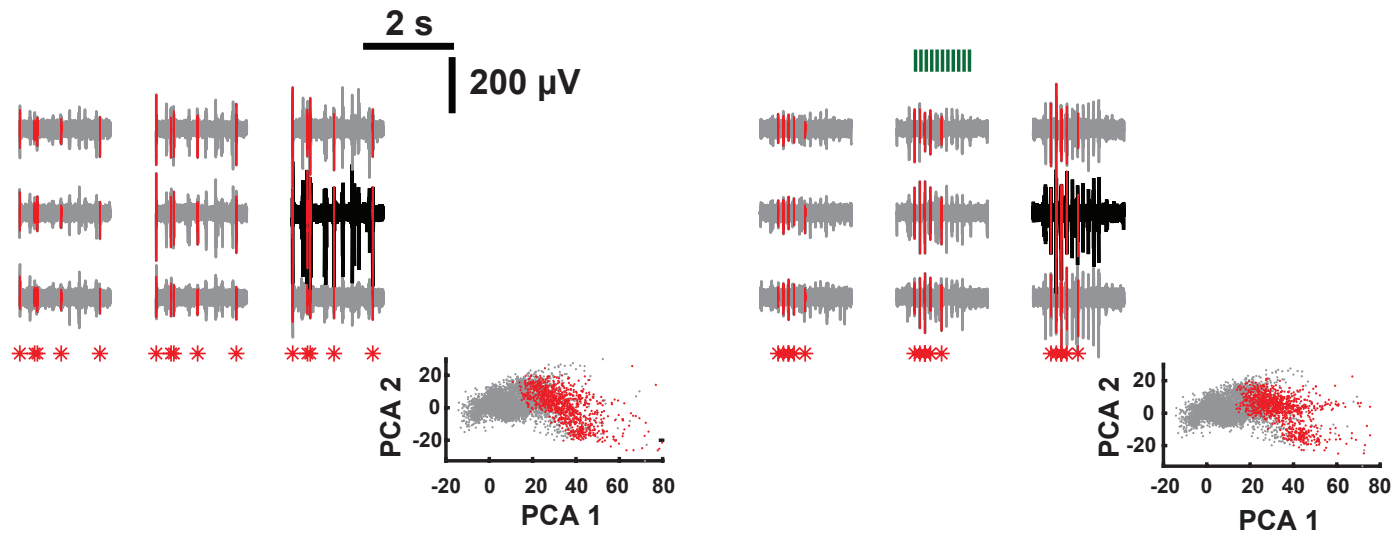

Supplementary Fig 6. Single-unit isolation quality of probe recordings during spontaneous and stimulus-driven conditions. A. Example mean single-unit waveforms in C and D for spontaneous (top, solid-filled) and stimulus-driven (bottom, dashed-filled) conditions. B. Peak-to-peak amplitude and unit quality of waveform across spontaneous and stimulus-driven condition. (Spontaneous (Vpp): VPm $=172$ $\pm 76.7 \mu \mathrm{V}, \mathrm{n}=9$ neurons, $\mathrm{S} 1=117 \pm 49.3 \mu \mathrm{V}, \mathrm{n}=11$ neurons; Stimulus-driven: $\mathrm{VPm}=171 \pm 75.2 \mu \mathrm{V}, \mathrm{n}=$ 7 neurons, $\mathrm{S} 1=118 \pm 48.2 \mu \mathrm{V}, \mathrm{n}=11$ neurons; Spontaneous $\left(\mathrm{SNR}_{\mathrm{Wf}}\right)$ : VPm $=6.06 \pm 1.62$ arbitrary unit (a.u.), $\mathrm{n}=9$ neurons, $\mathrm{S} 1=5.21 \pm 1.16$ a.u., $\mathrm{n}=11$ neurons; Stimulus-driven: VPm $=5.84 \pm 1.74$ a.u., $\mathrm{n}=$ 9 neurons, $\mathrm{S} 1=5.13 \pm 1.19$ a.u., $\mathrm{n}=11$ neurons, $\mathrm{N}=1$ mouse) C. Electrode configuration shown on the left. Sample voltage trace from VPm single unit with detected spikes (highlighted in blue) for spontaneous and stimulus-driven conditions. Inset (bottom): Waveform feature scatterplot showing spike clusters of the example unit and noise (gray, spikes from other whisker-responsive units detected on the same or neighboring channels). D. same as (C) but for S1 single unit. PCA 1 and PCA 2 plotted here are the two largest principle components of the example unit and other spikes are projected onto these dimensions. 